\title{
FAS research
}

FASresearch Sozialwissenschaftliche

Forschungsgesellschaft $\mathrm{mbH}$

Tel +43 (1) 3192655

Fax +43 (1) 3192657
Porzellangasse 2 / 34

1090 Wien, Austria

office@fas-research.com

www.fas-research.com

\section{Studie}

\section{zur Analyse bestehender Innovationsaktivitäten und Innovationspotentiale für den Standort Wien \\ Endbericht}

Überarbeitete Fassung, Wien, Juli 2015

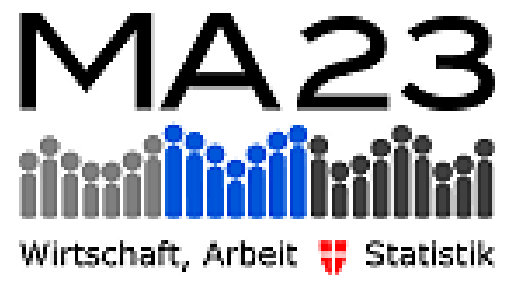




\section{Diese Studie entstand im Auftrag der Stadt Wien \\ MA 23 - Wirtschaft, Arbeit und Statistik}

\section{Inhaltsverzeichnis}

1. Ziele und innovationstheoretisches Modell der Studie ........................................................... 5

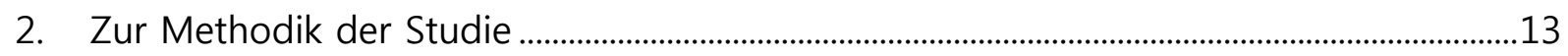

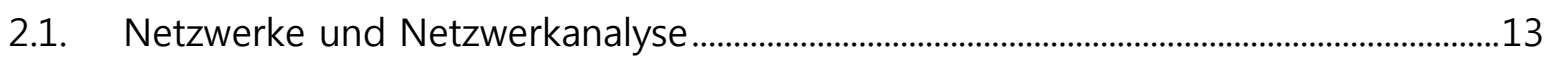

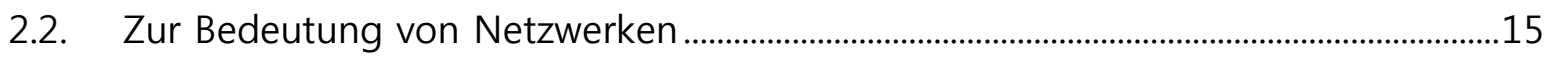

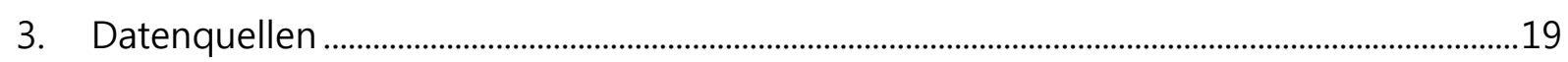

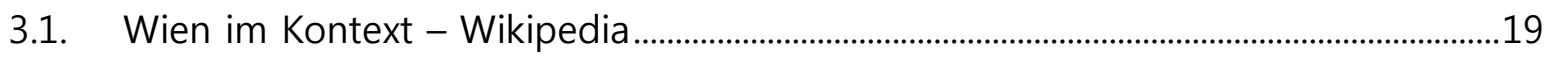

3.2. Wissenschaft, Forschung und Technologie ......................................................................19

3.2.1. Der Fonds zur Förderung der wissenschaftlichen Forschung (FWF) ...................19

3.2.2. Der Wiener Wissenschafts-, Forschungs- und Technologiefonds (WWTF)......20

3.2.3. Österreichische Forschungsförderungsgesellschaft (FFG) .......................................20

3.2.4. Rahmenprogramme der Europäischen Union.........................................................21

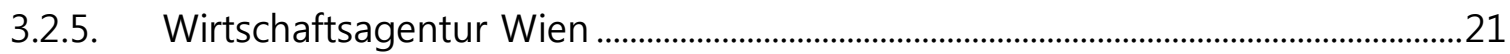

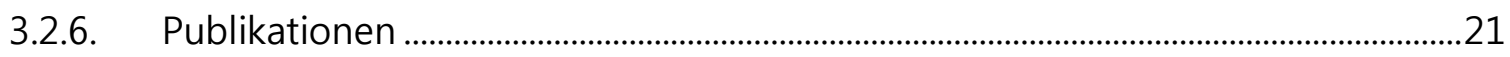

3.3. Ökonomischer Nutzen - Patente.....................................................................................22

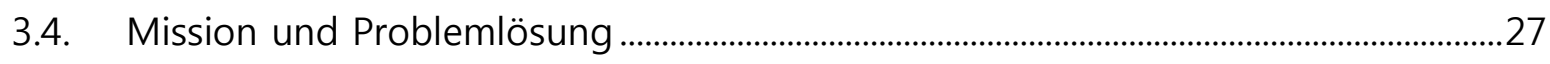

3.5. Generierung eines quellenübergreifenden Datensatzes für die Gesamtschau 2009

$-2013$ 28

4. Der Kontext der Stadt Wien .................................................................................................29

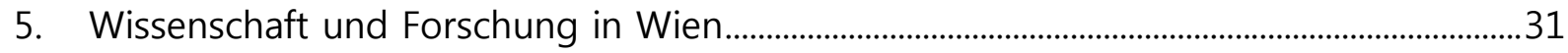


5.1. Die Wiener Partizipation an der Grundlagenforschung des FWF.

5.2. Wissenschaft und Forschung im WWTF 40

5.3. Publikationen in den Bereichen der Life Sciences und Technologie 44

5.4. Partizipation an EU-Rahmenprogrammen 53

5.5. Wiener Unternehmen in den Förderprogrammen der FFG

6. Die Wiener Patentlandschaft 65

6.1. Die Entwicklung der Patentzahlen Wiens 65

6.2. Die Technologiefelder der Patente 68

6.3. Das Alter der Patente in den Technologiefeldern . .76

6.4. Die Schlüsselspieler bei den Patentanmeldungen .77

6.5. Das Zitationsnetzwerk der Wiener Patentlandschaft. . .80

6.5.1. Das Zitationsnetzwerk der Patentklassen. . .80

6.5.2. Zitationsnetzwerk der Anmelder . .88

6.6. Das Patentportfolio Wiens. .96

7. Wiener Kreativwirtschaft und Social Entrepreneurship 102

8. Gesamtschau über die untersuchten Innovationsbereiche 105

8.1. Überblick 105

8.2. Die Verbinder von Exzellenz und Nutzen 115

8.3. Die Verbinder von Nutzen und Mission. 116

8.4. Die Verbinder von Exzellenz und Mission 118

9. Empfehlungen 125

10. Key-Deliverables der Studie 134

11. Abbildungs- und Tabellenverzeichnis 134 


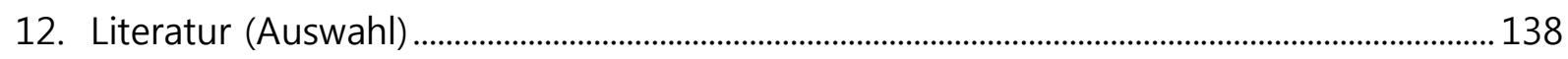




\section{Zlele und innovationstheoretisches Modell der Studie}

Das folgende Dokument stellt den Endbericht zur Studie "Analyse bestehender Innovationsaktivitäten und Innovationspotentiale für den Standort Wien" dar. Es handelt sich um eine überarbeitete Fassung der ersten Version vom März 2015.

Ziel der Studie ist die Analyse und Sichtbarmachung der zentralen Akteure des Innovationsgeschehens in Wien, ihrer Netzwerke, Bereiche und Technologien sowie ihres Momentums. Insgesamt ging es darum, bislang ungenutzte oder fehlende Potentiale sichtbar zu machen und auf Basis einer - hinsichtlich des Datenumfangs und der Datenquellen (FWF, WWTF, PubMed, IEEE, CORDIS, ESPACNET, DEPATISNET, Wiener Wirtschaftsagentur, FFG, FAS-Kreativwirtschaftsdatenbank, FAS-Social Entrepreneurdatenbank) - bislang einzigartigen Gesamtübersicht über die Innovationsökologie Wiens neue Chancen zur Verbesserung der Rahmenbedingungen, der Förderinstrumente und der gesamten Innovationskultur des Standortes herauszuarbeiten.

Das wesentliche Grundinteresse bestand in der Sichtbarmachung von Stärkefeldern, aber auch von Brüchen, Übergängen und "White Spaces" zwischen den einzelnen Sektoren des Innovationsgeschehens, die mit den Begriffen „Exzellenz", "Nutzen" und "Mission" zusammengefasst werden können. Diese bezeichnen die Währungen, um die es vorrangig in den drei Feldern der Wissenschaft, der Ökonomie und der Zivilgesellschaft geht, von deren Besitz die Position der Akteure in den Hierarchien der drei Felder abhängt und die die Verhaltensweisen der Akteure beeinflussen. ${ }^{1}$ Es gibt hier eine Vielzahl an Spannungsverhältnissen. Die Akteure konkurrieren nicht nur intern miteinander um diese Währungen (Wissenschaft: Publikationen, Drittmittel, Fördergelder etc.; Wirtschaft: Umsatz und Profit; Zivilgesellschaft: Erreichung gesellschaftspolitischer Ziele, mediale Präsenz, Mitgliederzahl,

\footnotetext{
${ }^{1}$ Der Begriff "Währung” wird hier ähnlich verwendet wie der des Kapitals bei Pierre Bourdieu. Gemäß seines Modells der sozialen Felder hängt die Position eines Akteurs vom Umfang und von der Zusammensetzung des Kapitals (ökonomisches, kulturelles und soziales Kapital) ab, über das er verfügt. Die Positionen im Feld wiederum beeinflussen die Haltungen und Verhaltensweisen der Akteure. Vgl. Bourdieu, Pierre (2000).
} 
Spenden), sondern auch die drei Währungen konkurrieren miteinander um gesellschaftliche Anerkennung (Grundlagenforschung vs. Anwendbarkeit, Wissenschaft vs. gesellschaftliche Relevanz usw.). Die White Spaces bestehen in den - netzwerkanalytisch gesprochen - strukturellen Löchern², d.h. fehlenden Beziehungen sowohl zwischen Akteuren innerhalb der drei genannten Innovationssphären als auch zwischen diesen Sphären selbst. Nicht überall aber sind zusätzliche Verbindungen sinnvoll, und vor allem sollen sie kein Selbstzweck sein - es geht um die Nutzung der Ressourcen der unterschiedlichen Währungen sowie der Ambiguitäten, die zwischen ihnen bestehen. Dem Konzept nach, das dieser Studie zugrunde liegt, sollte Innovationspolitik im weitesten Sinn inklusiv im Hinblick auf die drei Währungen sein. Die dahinterstehende Hypothese lautet, dass die Wiener Innovationsökologie dann resilient ${ }^{3}$ ist (im Sinne der Fähigkeit, immer wieder Zyklen der „schöpferischen Zerstörung" zu durchlaufen ${ }^{4}$ ), wenn die universitäre und die außeruniversitäre Grundlagenforschung, die Start-up Szene und etablierte Unternehmen, Staat, Verwaltung und Zivilgesellschaft im Sinne einer übergreifenden „Innovationsökologie” miteinander in Austauschbeziehungen stehen und nicht durch Fragmentierung, Isolation und Säulenbildung voneinander getrennt bleiben. Dahinter steht die aus der netzwerkanalytischen Innovationstheorie stammende Beobachtung, dass radikal Innovatives immer in Überlappungsbereichen von unterschiedlichen sozialen Kreisen (sogenannten „strukturellen Falten") entsteht, inkrementelle Innovationen hingegen innerhalb verdichteter sozialer Kreise (in "kritischen Massen") ihre Entstehungsbedingungen haben. ${ }^{5}$

\section{Abb. 1 - Die drel Währungen der Innovation}

\footnotetext{
2 Burt, Ronald S. (1995).

${ }^{3}$ Gunderson, Lance H./Holling, C.S. (2002).

4 Schumpeter, Joseph A. (2005).

${ }^{5}$ Vgl. Katzmair, Harald (2012) und Vedres, Balász/Stark, David (2010).
} 


\section{Ökonomischer Nutzen}

Unternehmen

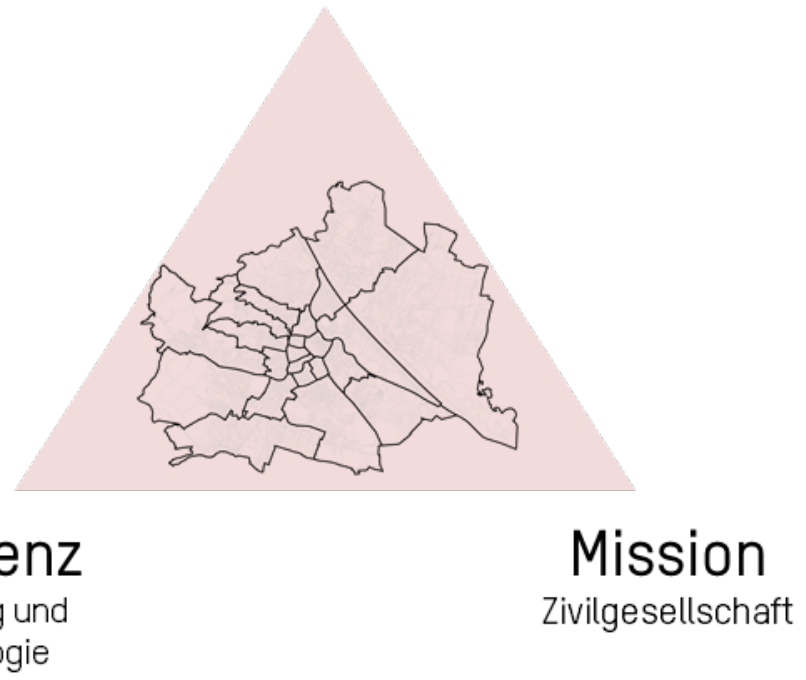

Mit den drei „Währungen" der Innovation sind unterschiedliche Weltbilder und Kulturen verbunden: ${ }^{6}$ Die hierarchische Kultur der Universitäten, großer Leitbetriebe sowie staatlicher Einrichtungen und intermediärer Institutionen, die tendenziell egalitäre Kultur der missionsorientierten Zivilgesellschaft und der Kreativwirtschaft, die individualistische Kultur der Entrepreneure und der Start-up Szene sowie die auf Autonomie bedachten Tüftler („Maker"), SchriftstellerInnen und KünstlerInnen, sind wesentliche Bestandteile einer erfolgreichen Innovationslandschaft, die auch mit unterschiedlichen Phasen des Innovationszyklus korrelieren und somit zur "Responsiveness" einer erfolgreichen Wiener Innovationsökologie zählen. Die Suche nach Neuem erfordert oft Introspektion und zeitweise Isolation (Autonomie) sowie eine Community und ein eingeschworenes, durch das Band der Freundschaft verbundenes Team, die durch die Höhen und Tiefen des Forschungsprozesses helfen (Egalitarismus). Die tatsächliche Umsetzung und Implementierung des Neuen benötigt Entrepreneure, "Abenteurer" und "Macher", die oft ähnlich den Goldgräbern von sehr individualistischen und Eigeninteressen geleitet sind (nicht zufällig wurde San Francisco von Goldgräbern gegründet, ein Spirit, der bis heute das Silicon Valley prägt); die Skalierung und das Wachstum und die reale Durchsetzung am Markt benötigt

\footnotetext{
6 Thompson, Michael/Ellis, Richard/Wildavsky, Aaron (1990) und Thompson, Michael (2008).
} 
jedoch Arbeitsteilung und kritische Masse und damit hierarchische, bürokratische Prinzipien der Führung und des strategischen Managements.

Die vor fast15 Jahren berühmt und prägend gewordenen drei T's von Richard Florida: Talent, Toleranz und Technologie ${ }^{7}$ verweisen auf diese Unterschiedlichkeit der Rollen, sofern wir Technologie mit "Hierarchie", Toleranz mit einer Kultur von "Autonomie" und „Egalitarismus” in Verbindung bringen können, und der Begriff des Talents auf „Individualismus" abzielt.

Gleichzeitig gibt es in jeder großen Organisation und damit in jeder größeren Stadt eine nicht unbeträchtliche Anzahl von demobilisierten Personen („disenfranchised scientists"), die sich aus welchen Gründen auch immer in ihren Organisationen am Abstellgleis befinden (oder gekündigt wurden), sich damit aus dem aktiven Innovationsprozess ausgeklinkt haben und für sich selber keine Chance für einen Neubeginn sehen (Fatalismus). Diese Gruppe bildet eine nicht zu unterschätzende schlummernde "strategische Reserve", eine Art "locked potential". Man sollte bei den "Talenten" eben nicht nur an den aufsteigenden, jungen, nach vorne strebenden Forscher/Entrepreneur denken, sondern gleichermaBen an die erfahrenen, älteren InnovatorInnen, die - würden sie „außerhalb” z.B. über buy-outs eine neue Chance bekommen - sehr wesentliche Beiträge für das lokale Innovationsgeschehen liefern könnten.

Ziel der vorliegenden Studie war vor diesem Hintergrund, ein besonderes Augenmerk auf die Schnittstellen und Übersetzungen zwischen den einzelnen Bereichen und kulturellen Rollen des Innovationszyklus zu legen und jene hybriden AkteurInnen mit hoher Nischenbreite zu identifizieren, die als „Broker" und "Übersetzer" fungieren. Die Ursache von sogenannten „White Spaces” liegt sehr häufig in der sozial-kognitiven Distanz zwischen unterschiedlichen Innovationskulturen, und zugleich sind es genau jene "White Spaces", wo Innovation entsteht: Der "Hierarchist" eines Leitbetriebs zum Beispiel trifft auf

\footnotetext{
${ }^{7}$ Florida, Richard (2002).
} 
den „Egalitaristen" der Co-Working Szene; der egalitäre, teamorientierte Gründer eines Creative Industry Start-ups trifft auf den individualistischen, rein von Geldinteressen geleiteten Investor; die status- und hierarchieorientierte Universitätsprofessorin trifft auf die unabhängigkeitsliebende, selbstbewusst-autonome Unternehmerpersönlichkeit; der enttäuschte, fatalistische Forscher/Manager am Abstellgleis einer großen Organisation trifft auf die idealistische Jungunternehmerin; der von Anlage- und Investitionsmöglichkeiten frustrierte "Reiche" trifft auf den idealistischen, kapitallosen Kreativen, etc.

Wenn Wien seine Innovationsstärke bewahren und weiterentwickeln will, benötigt es vor allem eine Arbeit an diesen „Begegnungen" und die Schaffung von neuen „Begegnungszonen", die auch der historischen Bedeutung von Wien als "Stadt der Begegnungen" einen neuen Sinn geben könnte. Am wissenschafts- und kulturhistorischen Höhepunkt von Wien waren es die jüdisch-bürgerlichen Salons, die diese Übersetzungsarbeit geleistet haben - die Frage stellt sich, wer oder was heute diese Rolle einnimmt. Die Studie möchte durch die Identifikation der Akteure und potentieller thematischer Rahmen hierfür Inputs liefern. Sie ist geleitet von der Überzeugung, dass wesentliche Chancen zur weiteren Stimulation von Innovation in Wien und in Europa in der Arbeit an der vorherrschenden „Innovationskultur" liegen. Im angelsächsischen Raum ist das Zusammenspiel von Exzellenz, Nutzen und Mission deutlich friktionsfreier, gerade das Silicon Valley demonstriert, wie sich über das Zusammenspiel von Universitäten, großen Unternehmen, Stiftungen/Non-Profits und staatlichen/militärischen Interessen eine deutlich kohärentere Innovationsökologie ausprägt als in Europa. Dass hierbei in den USA am Ende vor allem individualistische Interessen profitieren, ist offenkundig. Ein Wiener Weg, der vor allem auch gemeinwirtschaftliche, egalitäre Interessen berücksichtigt und aus passiven, demobilisierten "FatalistInnen“ wieder TeilnehmerInnen am politischen Leben und am Innovationsgeschehen macht, wäre eben nicht nur ein Wien angemessenes sozial- und gesellschaftspolitisches Projekt, sondern ein zutiefst innovationspolitisches Vorhaben (vom reinen Anwenden und Benutzen zum Machen und Gestalten). Die Vision einer Smart City sollte hierfür eigentlich einen genügend starken Rahmen abgeben, dass die unterschiedlichen 
Gruppen mit ihren unterschiedlichen Codes hier "ein Stück des Weges gemeinsam gehen"; an gesellschaftlichen, wirtschaftlichen und ökologischen Herausforderungen, die eine Smart City heute für morgen lösen muss, mangelt es ja nicht.

Am Ende wird es deshalb darum gehen, dass an den Schnittstellen des Innovationsprozesses mit seinen spezifischen Phasen und Rollen (in der Ökologie nennt man diese Übergangszonen „Ecotones") neue Räume und Formate initiiert bzw. bestehende Räume und Formate gestärkt werden; Ideen dafür werden im Empfehlungsteil der Studie vorgestellt. Denn solange die hierarchischen (Politik und Verwaltung), egalitären (Zivilgesellschaft und NGOs), individualistischen (Wirtschaft) und autonomen (Kunst) Kräfte sich nicht stärker als Teil eines gemeinsamen "Wiener Hauses” (Oikos) wahrnehmen, solange werden viele Bemühungen Bruchstück bleiben und im Sande verlaufen. Der Bedarf für Experimente und soziale Innovationen ist groß. Wien hat historisch bedeutsame Erfahrungen und könnte wieder einmal Vorreiterin für einen europäischen Weg sein, der die amerikanische, kalifornische Innovationskultur nicht einfach kopiert, sondern dem neoliberallibertären Projekt einen „Wiener Weg” entgegensetzt, in dem ein Haus gemeinsam bewirtschaftet wird, das groß und stark genug ist, um allen „Rollen” Platz zu bieten. 
Tabelle 1 - Übersicht: Wertehaltungen, Einstellungen und kulturelle Rollen einer Innovationsökologie

\begin{tabular}{|c|c|}
\hline Haltungen und Werte & Rollen-Beisplele \\
\hline AutonomistInnen & $\begin{array}{l}\text { - Einzelkünstler, Philosophen, Schriftsteller } \\
\text { - Bastler, Tüftler, Erfinder } \\
\text { - „Introverts” }\end{array}$ \\
\hline EgalitaristInnen & $\begin{array}{l}\text { - Missionsgetriebene NGOs } \\
\text { - Zivilgesellschaftliche Einrichtungen } \\
\text { - Start-ups, die aus Freundschaften oder Familienzusammen- } \\
\text { hängen hervorgehen } \\
\text { - Community basierte Start-ups im kreativen Umfeld }\end{array}$ \\
\hline IndividualistInnen & $\begin{array}{l}\text { - Klassische Entrepreneure } \\
\text { - Investoren, VCs } \\
\text { - Gründer und Eigentümer von Unternehmen }\end{array}$ \\
\hline HierarchistInnen & $\begin{array}{l}\text { - Universitätsangehörige } \\
\text { - Spitzenmanager in großen Leitbetrieben } \\
\text { - Personen aus öffentlichem Dienst und Verwaltung }\end{array}$ \\
\hline FatalistInnen & $\begin{array}{l}\text { - Demobilisierte, frustrierte Personen in großen Unternehmen } \\
\text { - „Disenfranchised Scientists“), generell Personen mit brach } \\
\text { - Drop-outs aus akademischen Karrieren } \\
\text { - Generell Personen, die nicht mehr an die Gestaltbarkeit und } \\
\text { - Veränderbarkeit der Welt glauben } \\
\text { "Das Heer der objektiv Ausgeschlossenen (Arbeitslose „Arme”, }\end{array}$ \\
\hline
\end{tabular}

In Fortführung von Thompson, Mlchael et al. (1990).

Neben der Beschreibung der einzelnen Währungen, Wertehaltungen und Rollen einer Innovationsökologie gilt es auch die Größen- und Zeitskalen, in denen die einzelnen Akteure operieren, zu modellieren: Grundsätzlich gilt, dass es ein Zusammenspiel zwischen "großen" und "kleinen" Akteuren sowie zwischen "SpezialistInnen" und "GeneralistInnen" 
benötigt, damit die Phasen des Innovationsprozesses ineinandergreifen können. Die Kleinen haben die Vorteile der Flexibilität und des Experiments, die Großen der Skalierung und der Pufferkapazitäten, die Spezialisten explorieren Nischen, die Generalisten „übersetzen" eher die unterschiedlichen Codes im Sinne von Einstellungen und Währungen. Hinzu kommt noch eine weitere Eigenschaft, die der "schnellen" und "langsamen" Bereiche: Die Veränderungsraten im Feld der "Computer Sciences" sind deutlich schneller als etwa im Feld der "Historical Sciences", so ist im Maschinen- und Anlagebau die Taktrate anders als im Feld der Datenübertragungen bzw. hat die Mikrobiologie andere Veränderungsraten als die Ökosystemforschung.

Dies gilt es zu berücksichtigen, denn die "White Spaces” in einer Innovationslandschaft bestehen eben nicht nur zwischen den beschriebenen Währungen und kulturellen Codes, sondern auch zwischen den "großen" und "kleinen" sowie "langsamen" und "schnellen" Bereichen. Generell können etwa die "langsameren" Bereiche, wie die Geistes- und Sozialwissenschaften, wertvolle Kontexte und gemeinsame Frames und Visionen vor allem für missionsgetriebene Innovation liefern, während die schnelleren Bereiche wie die Informations- und Lebenswissenschaften höhere Innovationspotentiale haben und in der Regel sozial auch durchlässiger sind (je schneller eine Technologie sich ändert, desto höher die Einstiegs- und Aufstiegschancen von "Außenseitern"). Aber nur in der Kombination entfalten beide ihre Stärken: Geschwindigkeit ohne Kontext ist "blind" und "orientierungslos", wie Orientierung ohne Umsetzung „leer" und „ohnmächtig" bleibt.

Generell bildet die Grundlage der vorliegenden Studie die Überzeugung, dass ohne ein Verständnis für die "langsamen Variablen" Wiens in Form seiner historischen Legacy (Wien als Stadt der Begegnung, Stadt der Musik, des Wassers, des Kaffeehauses, des Salons, Kabaretts, Stadt der sozialen Innovationen, des Gemeindebaus etc.) auch die Möglichkeiten der Mobilisierung von neuen Potentialen erschwert sind. Neue Ideen sollten daher auf ihre Bezogenheit zu diesen Grundmythen der Stadt überprüft werden. So wie San Francisco bis heute den kulturellen Code der Goldgräber nie abgelegt hat, hat Wien 
den Code der Begegnungsstätte von Ost und West, der Musik, der modernen positiven Wissenschaften („Wiener Kreis", Austrian School of Economics), der Psychoanalyse und Medizin etc. nie gänzlich abgelegt - ob uns dieses „alte” Bild von Wien nun gefällt oder nicht, es lebt auch heute noch fort. Auf diesen Mythen gilt es aufzubauen, oder sie gegebenenfalls zu erneuern und zu transformieren.

\section{Zur Methodlk der Studie}

\subsection{Netzwerke und Netzwerkanalyse}

Abgesehen von gängigen statistischen Methoden zur Datenauswertung und Ergebnisdarstellung wird in dieser Studie vor allem das Instrument der Sozialen Netzwerkanalyse (SNA) zur Beschreibung der Daten und der Informationen, die sie enthalten, verwendet. Bei der SNA handelt es sich um eine wissenschaftliche Methode zur empirischen Erfassung, Beschreibung, Visualisierung sowie Bewertung von Netzwerken. Unter einem Netzwerk versteht man eine abgegrenzte Menge von Knoten, die miteinander durch eine Menge von sogenannten Kanten verbunden sind. ${ }^{8}$ Die Begriffe "Knoten" und "Kanten" entstammen der Graphentheorie (neben der Matrixalgebra eines der Standbeine der SNA aus der Mathematik) und beziehen sich auf Entitäten, die miteinander durch Beziehungen verbunden sind. Handelt es sich dabei um soziale Akteure (Personen ebenso wie Kollektive) und um soziale Beziehungen (Kommunikation, Kooperation, Freundschaft, Verwandtschaft usw.), spricht man von sozialen Netzwerken. Das heißt, was ein Netzwerk ist, hängt allein von der Definition der Analytikerin oder des Analytikers ab. Wir finden Netzwerke nicht empirisch vor, sondern konstruieren sie mit Hilfe der Modelle der Netzwerkanalyse aus vorhandenen Daten über Akteure und Beziehungen. Die Allgemeinheit der Definition führt dazu, dass die Methoden der SNA auf alle möglichen Arten von Netzwerken (nicht nur auf soziale, sondern auch auf technische, semantische etc.) angewendet werden kön-

\footnotetext{
${ }^{8}$ S. Wasserman/K. Faust (1994), S. 28; D. Jansen (2003), S. 58; W. de Nooy/A. Mrvar/V. Batagelj (2011).
} 
nen. Nicht immer aber ist die Anwendung der SNA auch sinnvoll - wie bei allen wissenschaftlichen Instrumenten hängt es von den Fragestellungen ab, welche Methoden geeignet sind, sie zu beantworten. Wichtig ist vor allem, über eine Theorie und über Modelle darüber zu verfügen, was die sozialen Beziehungen antreibt, was ihr Inhalt ist und wie die Netzwerke entstehen, die Gegenstand der Analyse sind.

Die SNA als wissenschaftliche Disziplin kann auf eine lange Tradition verweisen, die mindestens bis zum Beginn des 20. Jahrhunderts zurückreicht. Ihre Ursprünge liegen unter anderem in der Soziologie (Georg Simmel), der Sozialpsychologie (Jacob L. Moreno als Begründer der Soziometrie, Fritz Heider mit der Balancetheorie, Kurt Lewin mit der Feldtheorie) und in der Sozialanthropologie (Strukturfunktionalismus). Deren Modelle wurden mit Methoden der Mathematik und Informatik kombiniert und zu Analyseinstrumenten fortentwickelt, die auch für die Untersuchung sehr großer Netzwerke geeignet sind. Von zentraler Bedeutung sowohl für die Auswertungsmethoden als auch für das Ausmaß an und den Zugang zu vorhandenen Daten sind die Entwicklung der Rechnerleistung von Computern, die Digitalisierung, neue Informations- und Kommunikationstechnologien (IKT) sowie das Internet.

Die Netzwerkanalyse wird als eine relationale Methode bezeichnet. ${ }^{9}$ Sie räumt der Analyse von Beziehungen den Primat gegenüber der Analyse von Merkmalen ein. Gängige empirische Methoden gehen so vor, dass sie Daten und Informationen über Merkmale von sozialen Akteuren sammeln und mit Hilfe statistischer Verfahren Zusammenhänge zwischen diesen Merkmalen zu entdecken versuchen. Modelle und Theorien zielen in der Folge darauf ab, unabhängige und abhängige Merkmale zu definieren sowie Kausalverhältnisse zwischen ihnen herzustellen (wie zum Beispiel zwischen Bildung und Einkommen oder sozialer Schicht und Meinungsbildung), um auf diese Weise soziale Tatsachen zu interpretieren. SNA hingegen zieht nicht (nur) Informationen über Attribute, sondern

\footnotetext{
${ }^{9}$ M. Emirbayer (1997).
} 
(auch) solche über Beziehungen heran, um Sachverhalte zu erklären (z.B. inwiefern und in welchem Maße hat die Netzwerkstruktur, in die ein Akteur eingebettet ist, Einfluss auf seine Verhaltensmuster und Denkweisen? Wie müssen die Netzwerke einer Organisation gestaltet sein, damit diese ihre Aufgaben und Funktionen besser erfüllen kann?). Dies unterscheidet SNA genuin von anderen wissenschaftlichen Instrumenten. Während ein Attribut oder Merkmal immer einem einzelnen Akteur (oder einem Kollektiv) zukommt, handelt es sich bei einer Beziehung um einen Sachverhalt, für den es mindestens zwei Akteure braucht. Dies hat zur Folge, dass es für die Datenerhebung, das Datenmanagement, die Analyse sowie die Darstellung der Ergebnisse spezifische Vorgehensweisen sowie eigene Analyseprogramme braucht.

\subsection{Zur Bedeutung von Netzwerken}

Grundsätzlich können Netzwerke als eine Art von sozialer Infrastruktur zur Allokation (Verteilung) von Ressourcen aufgefasst werden. ${ }^{10}$ Akteure erlangen kraft ihrer Beziehungen Zugang zu diesen Ressourcen, und das heißt, je besser die Netzwerkeinbettung eines Akteurs, desto besser sein oder ihr Zugang zu Ressourcen. Auf der Makroebene hängt das Vermögen eines Netzwerks, Ressourcen unter seinen Mitgliedern zu verbreiten, von seiner Strukturierung, das heißt von der Art der Verteilung der Beziehungen ab.

Mit Ressourcen sind dabei nicht nur Mittel wie Geld, Fertigkeiten, Wissen oder Informationen gemeint, die einem Akteur oder einer Organisation in einem bestimmten Innovationsbereich, in dem sie miteinander sowohl kooperieren als auch konkurrieren, Vorteile verschaffen. Ebenso handelt es sich um immaterielle Ressourcen wie Zuwendung, Aufmerksamkeit, Werte und Normen sowie um die Narrative, auf deren Basis soziale Identitäten (wie z.B. die in Tabelle 1 beschriebenen) entstehen. Im Zusammenhang mit den

${ }^{10}$ FAS.research (2005), S. 12. 
Ressourcen können verschiedene Formen von Kapital unterschieden werden, die sich Akteure in Netzwerken anzueignen versuchen ${ }^{11}$ :

- Ökonomisches Kapital (Besitz, Vermögen, Einkommen, d.h. finanzielle Mittel),

- Kulturelles oder Humankapital (Wissen, Fertigkeiten, Know-how und Informationen),

- Soziales Kapital (soziale Beziehungen und Netzwerke),

- Symbolisches Kapital (Anerkennung und Prestige).

Für die Netzwerkforschung ist insbesondere das soziale Kapital ein wichtiger Analysegegenstand, der sich mit der Frage beschäftigt, was eigentlich "gute" Beziehungen und Netzwerke von „weniger guten" unterscheidet.

Insgesamt geht es also darum, Netzwerke als Strukturen von „Kanälen“ zu modellieren, durch die soziale Ressourcen "fließen" oder getauscht werden, und die auf diese Weise die Akteure, die in sie eingebettet sind, am Innovationsgeschehen teilhaben lassen. Damit ist auch erklärt, dass Netzwerke niemals „von alleine” entstehen können. Es müssen bestimmte Voraussetzungen erfüllt sein, anders gesagt: Es müssen Ressourcen vorhanden sein, die durch die Netzwerke "fließen" können, damit Beziehungen überhaupt eingegangen werden; allerdings muss es sich nicht unbedingt um materielle Ressourcen handeln. Dieser Aspekt - dass Netzwerke von Voraussetzungen abhängig sind - wird sowohl in der Theorie (Netzwerkforschung) als auch in der Praxis (Netzwerkmanagement) häufig vernachlässigt. Manchmal wird angenommen, dass allein der (zusätzlich vielleicht noch von zentraler Stelle verordnete) Zusammenschluss von Akteuren dafür sorgt, dass es z.B. zu Zusammenarbeit und Produktion von Innovationen kommt. Davon kann aber zumeist nicht ausgegangen werden; vielmehr betrachten wir die Netzwerke selbst als einen Effekt von Aktivität, Produktion und Ressourcenfluss.

\footnotetext{
11 Vgl. stellvertretend Pierre Bourdieu (2005).
} 
Ein wichtiger Bestandteil dieser Studie sind die Netzwerkkarten, die erstellt werden, um die Strukturen der Verbindungen zwischen den Akteuren sichtbar zu machen. ${ }^{12}$ SNA stellt eine Vielzahl an Methoden zur Verfügung, mit deren Hilfe Schlüsselspieler, Schlüsselbeziehungen, Cluster von Akteuren sowie die bereits angesprochenen Beziehungslücken (strukturelle Löcher) identifiziert und abgebildet werden können. Netzwerkanalyse ist ein recht allgemeines Instrument zur Deskription von Beziehungsmustern. Nicht immer muss es sich um soziale Akteure (Menschen, Organisationen) und soziale Beziehungen (Kooperation, Ressourcentransfer usw.) handeln, es können z.B. auch Begriffe sein (die Wissenschafts- und Technologierfelder, in denen sich die Innovationsaktivitäten der Stadt Wien abspielen) und ihr gemeinsames Sich-Zeigen in einem Dokument, wie wir noch sehen werden. Um eine Netzwerkkarte lesen zu können, ist die Information darüber unabdingbar, um welche Akteure es sich handelt, und was die Beziehungen bedeuten. Diese Information findet sich jeweils in den Bildunterschriften unter den Netzwerkkarten. Es gibt ein paar ganz allgemeine Fragestellungen, die den Betrachter bei der Interpretation einer Netzwerkkarte helfen:

- Wer sind die Akteure und was sind die Beziehungen?

- Auf welchen Zeitraum bezieht sich die Darstellung?

- Handelt es sich um ein zusammenhängendes Netzwerk oder gibt es voneinander getrennte Netzwerkbereiche oder Akteure (Isolate)?

- Wer befindet sich eher im Zentrum, wer an der Peripherie des abgebildeten Netzwerks? ${ }^{13}$ Wer erscheint als besser, wer als weniger gut vernetzt (häufig durch die Größe der Netzwerkknoten dargestellt)?

- Welche Beziehungen erscheinen als besonders wichtig (durch die Stärke der Linien oder Pfeile symbolisiert)?

\footnotetext{
12 Krempel, Lothar (2005).

${ }^{13}$ Es muss betont werden, dass Netzwerke tendenziell unendlich sind, und dass sich die Aussage darüber, ob ein Akteur sich im Zentrum oder an der Peripherie befindet, immer nur auf das im konkreten Fall untersuchte und sichtbare Netzwerk beziehen kann.
} 
- Gibt es Cluster von Akteuren oder Netzwerkknoten, die einander hinsichtlich der Beziehungen, über die sie verfügen, ähneln und darum nahe beieinander liegen und/oder in der gleichen Farbe dargestellt sind? 


\section{Datenquellen}

Um die Innovationslandschaft Wiens zu beschreiben, zieht die vorliegende Studie zehn verschiedene Datenquellen heran und verwendet eine Vielzahl an Modellen und Analysemethoden. Am Ende ging es darum, aus den verschiedenen Datenquellen eine Datenbank zu generieren, in der für jeden Wiener Akteur, der sich darin befindet (Unternehmen, Universitäten, außeruniversitäre Forschung, Zivilgesellschaft, Politik und Verwaltung), eingetragen ist, in welchem Förderinstrument bzw. in welchem Innovationsbereich er vorkommt, und aus der eine Vielzahl an Netzwerken erzeugt werden kann, die mittels statistischer Verfahren und netzwerkanalytischer Instrumente ausgewertet werden. Die Datenquellen, Förderinstrumente und Bereiche im Einzelnen sind: Wikipedia, FWF, WWTF, FFG, EU-Rahmenprogramme, Wirtschaftsagentur Wien, Deutsches und Europäisches Patentamt, Kreativwirtschaft und Social Entrepreneurship.

\subsection{Wien im Kontext - Wikipedia}

Aus Wikipedia wurden all jene Artikel extrahiert, die den Begriff „Vienna” enthalten - insgesamt handelt es sich um 30.216 Einträge. Zusätzlich wurden der Titel der Artikel sowie die monatlichen Zugriffszahlen durch BearbeiterInnen von Jänner bis Dezember 2014 erhoben und gespeichert. Dies ermöglicht die Identifikation von Artikeln, in deren Kontext Wien auftaucht, sowie die Berechnung des Momentums der Themen, d.h. der Entwicklung der Zugriffszahlen, die im Kontext von Wien im Jahr 2014 besonders virulent waren.

\subsection{Wissenschaft, Forschung und Technologie}

3.2.1. Der Fonds zur Förderung der wissenschaftlichen Forschung (FWF) 
Aus der Projektdatenbank des FWF ${ }^{14}$ wurden die im Zeitraum vom 01.01.1995 bis zum 15.10.2014 bewilligten Projekte extrahiert. Insgesamt handelt es sich um 11.706 Projekte, darunter 6.385 (54,5\%) mit einer Beteiligung von insgesamt 151 Akteuren aus Wien. Die Forschungsbereiche der Projekte sind in Form der Österreichischen Systematik der Wissenschaftszweige 2012 auf 3-Steller-Ebene klassifiziert.

Tabelle 2 - Aufbau der ÖFOS 2012 anhand des Belsplels MedlzInlsche Blotechnologle (Code 3040)

\begin{tabular}{llll} 
Code & Stellen & Ebene & Bezeichnung \\
\hline 3 & Einsteller & Hauptgruppe & Humanmedizin, Gesundheitswissenschaften \\
304 & Dreisteller & Gruppe & Medizinische Biotechnologie \\
3040 & Viersteller & Untergruppe & Medizinische Biotechnologie
\end{tabular}

3.2.2. Der Wiener Wissenschafts-, Forschungs- und Technologiefonds (WWTF)

Die Projektdatenbank des WWTF ${ }^{15}$ enthält mit Stichtag 05.02.2015 insgesamt 258 Projekte unter Beteiligung von 66 Institutionen und Unternehmen aus Wien. Der Inhalt der geförderten Projekte ist durch die Bezeichnung des jeweiligen Programms beschrieben:

- Universitäts-Infrastruktur-Programm

- Life Sciences

- "Mathematik und..."

- Informations- und Kommunikationstechnologien

- Geistes-, Sozial- und Kulturwissenschaften

- Kognitionswissenschaften

\subsection{3. Österreichische Forschungsförderungsgesellschaft (FFG)}

Die Daten über jene Wiener Unternehmen, die in den Jahren 2012, 2013 und 2014 eine Förderung der FFG erhalten haben, wurden uns von der Wirtschaftsagentur Wien zur Verfügung gestellt. Darin enthalten sind 980 Akteure, die ihren Sitz in Wien haben, sowie die

\footnotetext{
${ }^{14}$ https://pf.fwf.ac.at/de/wissenschaft-konkret/project-finder, abgerufen am 15.10. 2014.

15 http://www.wwtf.at/projects/project_database/, abgerufen am 05.02.2015.
} 
Kennzeichnung der Wirtschafts-, Forschungs- und Technologiefelder in Form eines Subject Index Codes, der insgesamt 44 Kategorien enthält. Wichtig ist darauf hinzuweisen, dass die Liste nur Unternehmen enthält, und keine an FFG-Projekten beteiligte Universitäten oder außeruniversitäre Forschungseinrichtungen.

\subsubsection{Rahmenprogramme der Europäischen Union}

Die Datengrundlage für die Erfassung der EU-geförderten Projekte umfasst für den Zeitraum 1981 bis 2014 insgesamt 100.892 Projekte, die vollständig aus der CORDIS-

Datenbank der Europäischen Kommission extrahiert wurden. ${ }^{16}$ Für den Analysezeitraum 2002 bis 2014 (6. und 7. EU-Rahmenprogramm) sind in CORDIS 2.654 Projekte verzeichnet, an denen insgesamt 601 Akteure mit Sitz in Wien beteiligt waren.

\subsubsection{Wirtschaftsagentur Wien}

Die von der Wirtschaftsagentur Wien geförderten Projekte wurden dem Datenkatalog des Open Government Wien entnommen ${ }^{17}$. Dieser umfasst für den Zeitraum 2002 bis 2013 insgesamt 972 geförderte Projekte (inklusive jener der wienwin-Initiative). Die Beschreibung des Inhalts der Programme erfolgt durch eine Klassifikation mit sechs Kategorien: Informations- und Kommunikationstechnologie, Ingenieur- und Naturwissenschaften, Medien, Life Sciences, Energie und Umwelt sowie Geistes-, Sozial- und Wirtschaftswissenschaften.

\subsubsection{Publikationen}

Um zwei Schwerpunkte der Innovationslandschaft Wiens im Hinblick auf den Publikationsoutput - Life Sciences und Technologie - zu charakterisieren, wurden in den Publikationsdatenbanken Pubmed und IEEE die Publikationen mit Wiener Beteiligung in den Zeiträumen 2010 bis 2014 bzw. 2002 bis 2013 identifiziert und extrahiert. Insgesamt han-

\footnotetext{
16 http://cordis.europa.eu/home_de.html, abgerufen am 09.01.2015.

17 https://open.wien.gv.at/site/datenkatalog/, abgerufen am 20.02.2015.
} 
delt es sich dabei um 11.316 (Pubmed) sowie 5.364 (IEEE) Publikationen. Die Auswertung erfolgte jedoch nicht im Hinblick auf die Akteure, sondern auf die Forschungs- und Technologiebereiche, in denen publiziert wurde. Diese liegen in Form der sogenannten SCOPUS-Klassifikation (Pubmed) bzw. von Schlagworten (IEEE) vor, die sich auf die Journals beziehen, in denen die Publikationen erscheinen.

Bei Pubmed ${ }^{18}$ handelt es sich um eine Meta-Datenbank mit Publikationen aus den Bereichen Medizin, Zahnmedizin, Veterinärmedizin, öffentliches Gesundheitswesen, Psychologie, Biologie, Genetik, Biochemie, Zellbiologie, Biotechnologie und Biomedizin. Sie wurde vom National Center for Biotechnology Information (NCBI) des National Institute of Health (NIH) in den USA entwickelt. Pubmed ist die umfassendste aller medizinischen Publikationsdatenbanken. Scopus ist eine Zitations- und Abstractdatenbank für wissenschaftliche Publikationen, die auch eine Klassifikation der Journals nach Fachbereichen enthält. Diese dient bei den folgenden Analysen zur Beschreibung der medizinischen Felder, in denen die erhobenen Wiener Publikationen angesiedelt sind.

IEEE $^{19}$ ist das Institute of Electrical and Electronics Engineers, die weltweit größte Fachgesellschaft von Ingenieurinnen und Ingenieuren in den Bereichen Elektrotechnik und Informationstechnologien mit Sitz in New York. IEEE betreibt unter anderem eine OnlineBibliothek mit den Publikationen der über 100 verbandseigenen Fachzeitschriften. Den Beiträgen in diesen Journalen und bei IEEE-eigenen Konferenzen wird eine hohe wissenschaftliche Relevanz und fachliche Qualität zugeschrieben.

\section{3. Ökonomischer Nutzen - Patente}

Patente verhindern, dass neue technische Erfindungen, Produkte oder Verfahren von jemand anderem als dem Patentinhaber produziert und/oder vermarktet werden, wobei der

\footnotetext{
${ }^{18}$ http://www.ncbi.nlm.nih.gov/pubmed.

19 https://www.ieee.org/index.html.
} 
Patentschutz räumlich definiert und zeitlich begrenzt ist. Patentierungen sind dem Bereich des ökonomischen Nutzens zuzuordnen, und die Patentanalysen dienen in dieser Studie dazu, die Aktivitäten Wiener Akteure im Zusammenhang mit der wirtschaftlichen Verwertung darzustellen, ohne dass damit gesagt werden soll, dass die Verwertung damit vollständig beschrieben ist. Die Analyse von Patentdaten ist mit zahlreichen Schwierigkeiten und Herausforderungen verbunden ${ }^{20}$, insbesondere was die Beschreibung und Interpretation der Patentklassen betrifft, in denen die Patentierungen stattfinden. Andererseits liefern Patentdaten umfassende Informationen über die Verwertung von F\&E-Aktivitäten in zahlreichen Technologiefeldern, und zu diesem Zweck werden sie auch in dieser Studie genutzt.

Als Datenquellen für die Patentstudien dienen uns die Patentdatenbanken Espacenet des Europäischen Patentamtes (European Patent Office - EPO) und Depatisnet des Deutschen Patent- und Markenamtes (DPMA). Espacenet deckt Patente auf europäischer und globaler Ebene ab; Depatisnet wurde zusätzlich herangezogen, um die Patente zu identifizieren, deren Schutz sich auf Österreich beschränkt.

Abb. 2 - Auszug aus der Espacenet-Patentdatenbank mit einem Patent von Slemens Österreich

\footnotetext{
20 Vgl. z.B. Unterlass, Fabian/Hranyai, Kathrin/Reinstaller, Andreas (2013): Patentindikatoren zur Bewertung der erfinderischen Leistung in Österreich. Vorläufiger technischer Bericht. Österreichisches Institut für Wirtschaftsforschung, Wien 2013.
} 


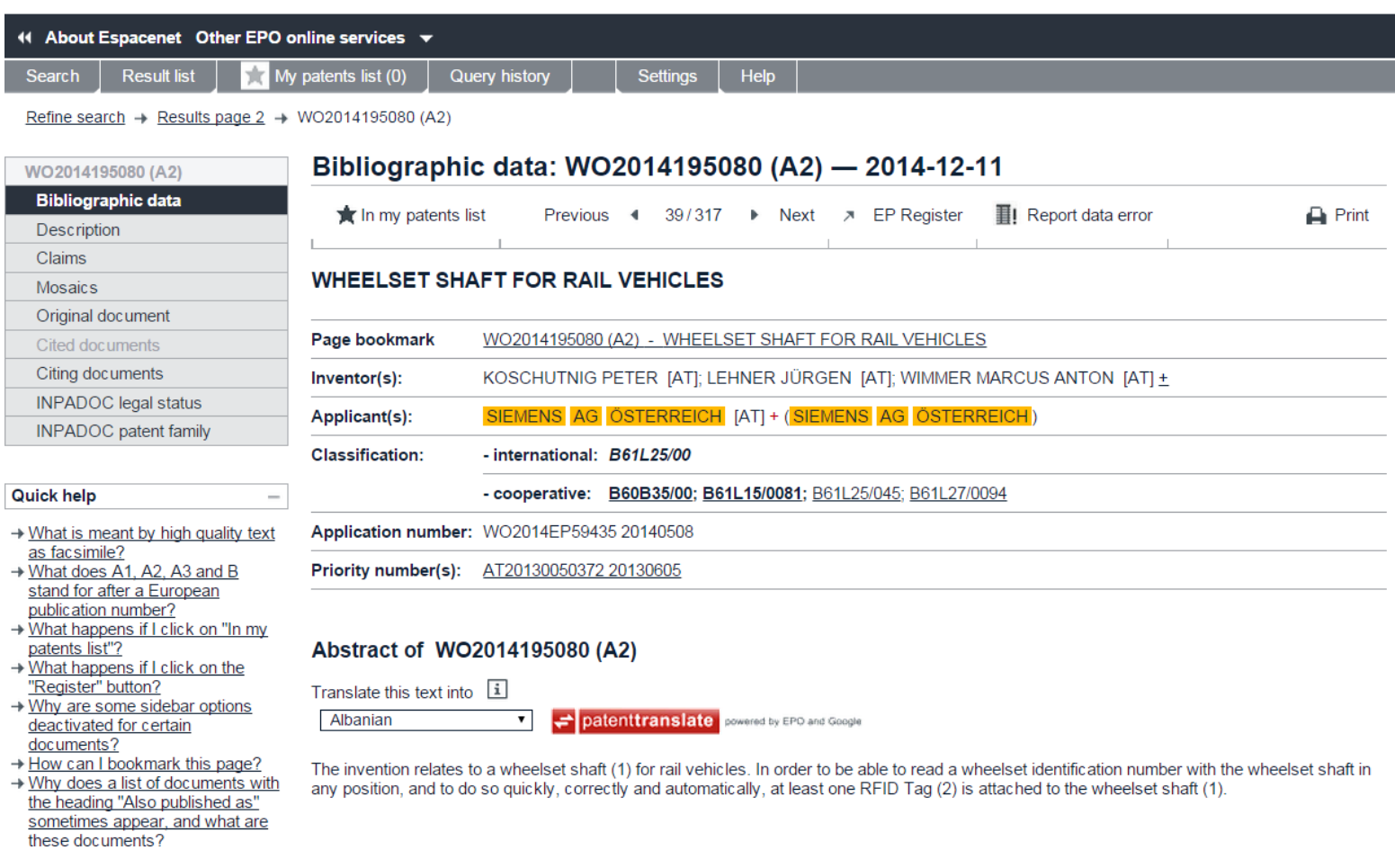

Espacenet und Depatisnet enthalten jeweils mehrere Millionen Patente. Für die Studie wurden all jene Patente identifiziert, deren Anmelder aus Österreich stammen (d.h. mit einer österreichischen Adresse versehen sind). Für diese Patente wurden die in den Datenbanken enthaltenen Informationen erhoben und zur weiteren Analyse aufbereitet, dabei vor allem:

- Patent-ID zur Identifikation

- Bezeichnung (Titel) des Patents

- Anmelder (Personen, Unternehmen, sonstige Institutionen; national und international)

- Adresse des Anmelders

- IPC-Patentklassen, die die Technologiefelder bezeichnen, in denen die Patentierung erfolgt ist

- Jahr der Anmeldung

Aus diesen Daten können vor allem folgende Netzwerke generiert werden:

- Netzwerk der Anmelder, die durch Patente verbunden sind 
- Netzwerk der Anmelder, die in denselben Patentklassen (Technologiefeldern ) patentieren

- Netzwerk der Patentklassen (Technologiefelder), die durch Patente verbunden sind

Die Datenerhebung bei den Patenten bezog sich auf die Jahre 1979 bis 2014. (In den Patentdatenbanken waren die Patente in digitalisierter Form seit 1979 für uns zugänglich.) Für diesen Zeitraum wurden insgesamt 62.966 Patente mit österreichischer Beteiligung erhoben (Depatisnet plus Espacenet). An ihnen waren in Summe 14.138 Anmelder aus Österreich beteiligt.

Innerhalb dieser Menge finden sich zusammengenommen 16.161 Patente unter Beteiligung von 2.441 Anmeldern aus Wien. Damit ist die Adresse des Firmensitzes gemeint, die in den Patentdaten enthalten ist. Es muss darauf hingewiesen werden, dass der Firmensitz natürlich nicht immer mit einem Produktionsstandort zusammenfällt; das ist bei der Interpretation der Ergebnisse zu beachten.

Über diese Daten hinaus wurden für alle österreichischen Patente aus Espacenet (internationale Gültigkeit) die Zitationsbeziehungen erhoben.

\section{Abb. 3 - Patentzltatlonen: Zltatlonsrlchtung und Wlssenstransfer}

$\begin{gathered}\text { Backward } \\ \text { Citation }\end{gathered}$
$\begin{gathered}\text { Wiener } \\ \text { Patentnetzwerk }\end{gathered}$ $\begin{gathered}\begin{array}{c}\text { Forward } \\ \text { Citation }\end{array} \\ \text { Output }\end{gathered}$


In vielen Fällen zitiert ein angemeldetes Patent ein anderes Patent und nimmt auf diese Weise auf früher entstandenes Know-how Bezug (Backward Citation, Input an Wissen). Ebenso kann ein Patent von anderen Patenten zitiert werden und liefert insofern einen Output an Wissen (Forward Citation).

Von den erfassten 62.966 österreichischen Patenten sind 25.745 in Zitationsbeziehungen eingebunden (40,8\%), die zu insgesamt 55.328 internationalen Patenten führen.

Innerhalb dieses Zitationsnetzwerks finden sich 7.481 Wiener Patente (von den genannten 16.161; d.h. 46,3\% der Wiener Patente zitieren andere Patente und/oder werden von anderen Patenten zitiert). In der 1-Schritt-Umgebung der Wiener Patente finden sich 21.709 Patente mit Anmeldern außerhalb Wiens (309 nationale und 21.318 internationale).

Durch die Verknüpfung über die Patent-IDs können aus den Zitationsdaten zwei weitere Zitationsnetzwerke generiert werden:

- Netzwerk der Anmelder, die miteinander durch Zitationen verbunden sind (wer bezieht sich auf wen),

- Netzwerk der Patentklassen, die miteinander durch Zitationen verbunden sind (adjazente Technologiefelder und Wissens-Wertschöpfungsketten).

Um die Technologiefelder zu kennzeichnen, in denen die Patentierungen erfolgen, wird im Allgemeinen die Internationale Patentklassifikation (IPC) verwendet. Diese ist folgendermaßen aufgebaut:

Tabelle 3 - Aufbau der IPC Patentklassifikation

\begin{tabular}{|c|c|c|}
\hline IPC-Aufbau & Ebene & Bezeichnung (Beispiele) \\
\hline A & Sektion & Täglicher Lebensbedarf \\
\hline A61 & Klasse & Medizin oder Tiermedizin; Hygiene \\
\hline A61K & Unterklasse & Präparate für medizinische, zahnärztliche oder kosmetische Zwecke \\
\hline A61K 39/00 & Gruppe & $\begin{array}{l}\text { Medizinische Präparate, die Antigene oder Antikörper enthalten (Impf- } \\
\text { stoffe) }\end{array}$ \\
\hline A61K 39/139 & Untergruppe & Mumps- oder Masernvirus \\
\hline
\end{tabular}


Die Analysen der Technologiefelder erfolgen in dieser Studie auf der Ebene der Klassen und der Unterklassen. Insgesamt sind den Wiener Patenten 545 Patent-Unterklassen zugeordnet.

\subsection{Mission und Problemlösung}

Um einen Innovationsbereich, der missionsgetrieben und eng mit der Zivilgesellschaft verflochten ist, wenigstens zum Teil abzubilden, greifen wir auf FASresearch-eigene Datenbestände zurück. Diese wurden mit Hilfe der referentiellen Netzwerkanalyse generiert, die auf ExpertInnengespräche mittels Schneeballverfahren basiert. Dieses läuft so ab, dass bei einer gewissen Anzahl von bereichsspezifisch und regional verteilten Starting Points (UnternehmerInnen, Organisationen) mit der Befragung begonnen wird. Die Starting Points werden gebeten, Personen, Firmen und/oder Organisationen zu nennen, die in einem bestimmten Bereich (wie etwa der Kreativwirtschaft) eine wichtige Rolle spielen sei es z.B. als ExpertInnen, als etablierte Player oder als Akteure, die Einfluss auf politische oder finanzielle Rahmenbedingungen haben. Die genannten Personen werden wiederum nach der gleichen Art und Weise befragt. Dies geht über mehrere Runden, so lange, bis der Anteil der Personen, die durch die Nominationen neu hinzukommen (und nicht bereits früher genannt wurden) unter einen bestimmten Wert pro Befragungsrunde fällt (in etwa 20 zu 80). Die daraus gewonnenen Daten ergeben Netzwerke von Personen, die miteinander durch Nominationsbeziehungen verbunden sind. Sie stellen keine repräsentative Stichprobe (im Sinne einer gleichen oder angebbaren Wahrscheinlichkeit, in die Stichprobe zu gelangen) dar; Schneeballverfahren zielen vielmehr auf die Identifikation von im weitesten Sinne "bekannten" sozialen Akteuren ab.

Die für die Bereiche Kreativwirtschaft sowie Social Entrepreneurship erhobenen Daten enthalten insgesamt 807 Unternehmen und Organisationen mit Sitz in Wien. 


\subsection{Generierung eines quellenübergreifenden Datensatzes für die Gesamt-} schau 2009 - 2013

Ursprünglich war ein wichtiges Ziel dieser Studie die Herstellung einer Datenquellenübergreifenden Ontologie zur Charakterisierung der Innovationsaktivitäten in unterschiedlichen Bereichen, um beispielsweise den Output in der Grundlagenforschung (Exzellenz) mit jenem der Patente (ökonomische Verwertung) vergleichen zu können. Die umfangreichen Bemühungen in dieser Hinsicht haben bislang zu keinem ausreichend befriedigenden Ergebnis geführt. Die Zuordnung von Technologieklassen zu Projekten oder Publikationen aufgrund der Beschreibungen der Abstracts mit Hilfe lernender, automatisierter textanalytischer und semantischer Technologien (Zerlegung der Texteinheiten in n-grams und Zuordnung mittels mathematischer Ähnlichkeitsmodelle) beispielsweise erwies sich vor allem auf der feinsten Ebene als nicht in ausreichendem Maße zufriedenstellend. Zwar gelang eine korrekte Zuordnung in manchen Technologiebereichen in bis zu $80 \%$ der Fälle, aber dies ist nicht ausreichend, um die verschiedenen Felder valide miteinander vergleichen zu können. Aus diesem Grund wurde für die Studie ein Datensatz erzeugt, in dem nicht die Forschungs- und Technologieklassen, sondern die Akteure die unterschiedlichen Datenquellen miteinander verbinden, deren Charakterisierung dazu dient, die Wiener Innovationslandschaft zu beschreiben. Es handelt sich nicht eine Vollerhebung und auch nicht um eine Stichprobe im statistischen Sinn, sondern um die Summe aller Organisationen und Unternehmen, die für den Zeitraum 2009 bis 2013 laut der uns vorliegenden Daten in den oben aufgelisteten Innovationsbereichen und Förderinstrumenten aufscheinen. 


\section{Der Kontext der Stadt Wien}

Mit jeder Stadt werden bestimmte Vorstellungen assoziiert, und diese Vorstellungen bilden zusammen einen narrativen Kontext, in den sie eingebettet ist und der sowohl positive als auch negative Aspekte umfasst. Wir haben versucht, diesen Kontext anhand der Wikipedia-Artikel zu charakterisieren, in denen der Begriff "Vienna” vorkommt - hier die Titel dieser Artikel gereiht nach den Zugriffszahlen bzw. deren Steigerung 2014.

\section{Abb. 4 - Wlklpedla-Artlkel mlt „Vlenna“ nach der Anzahl der Zugrlffe 2014}

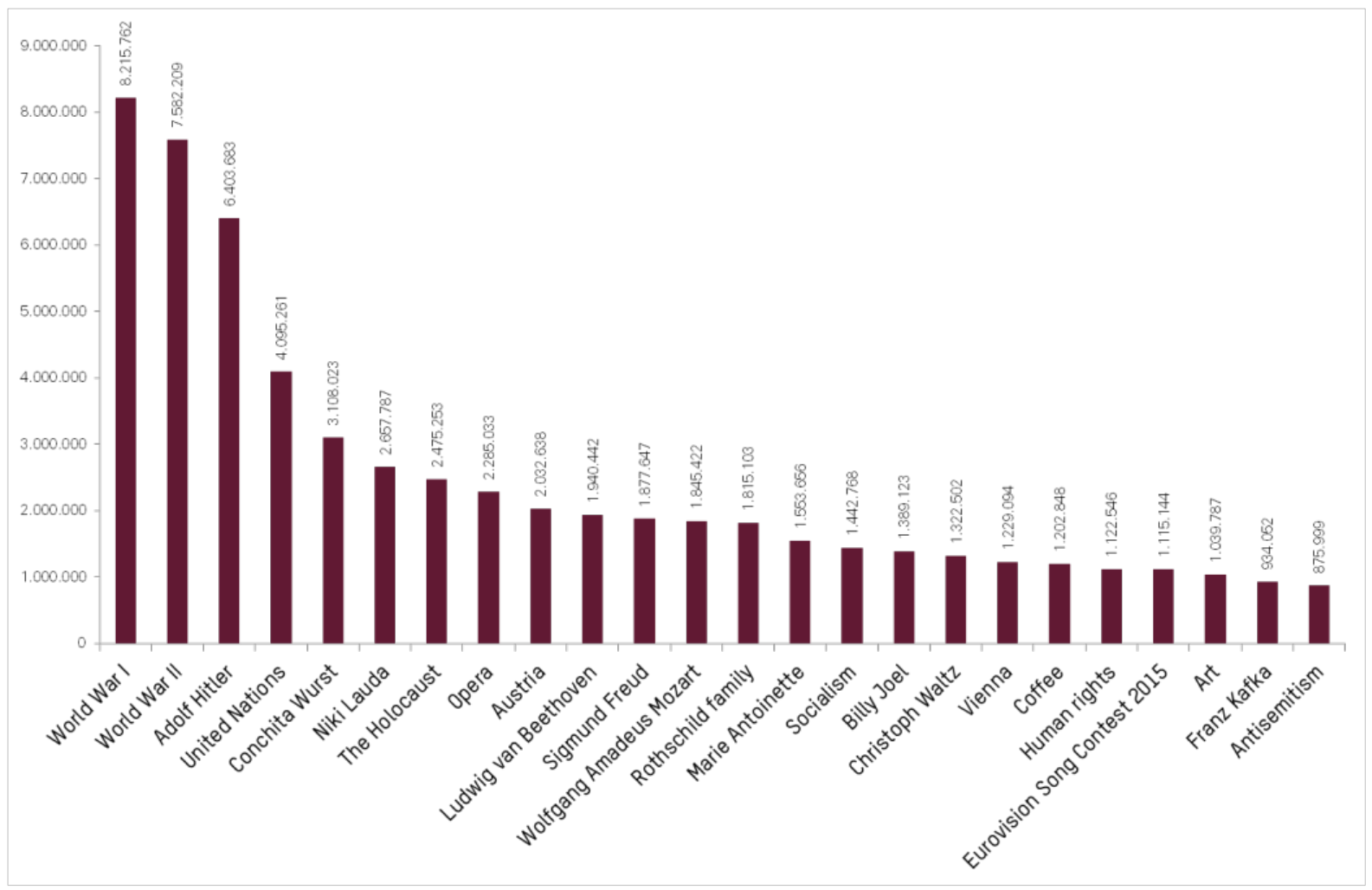

Datenquelle: Wikipedia. N = 30.216 Artikel. Analyse: FASresearch 


\section{Abb. 5 - „Vienna“-Wikipedia-Artikel nach der durchschnittlichen Zunahme an monatlichen Zugriffen 2014}

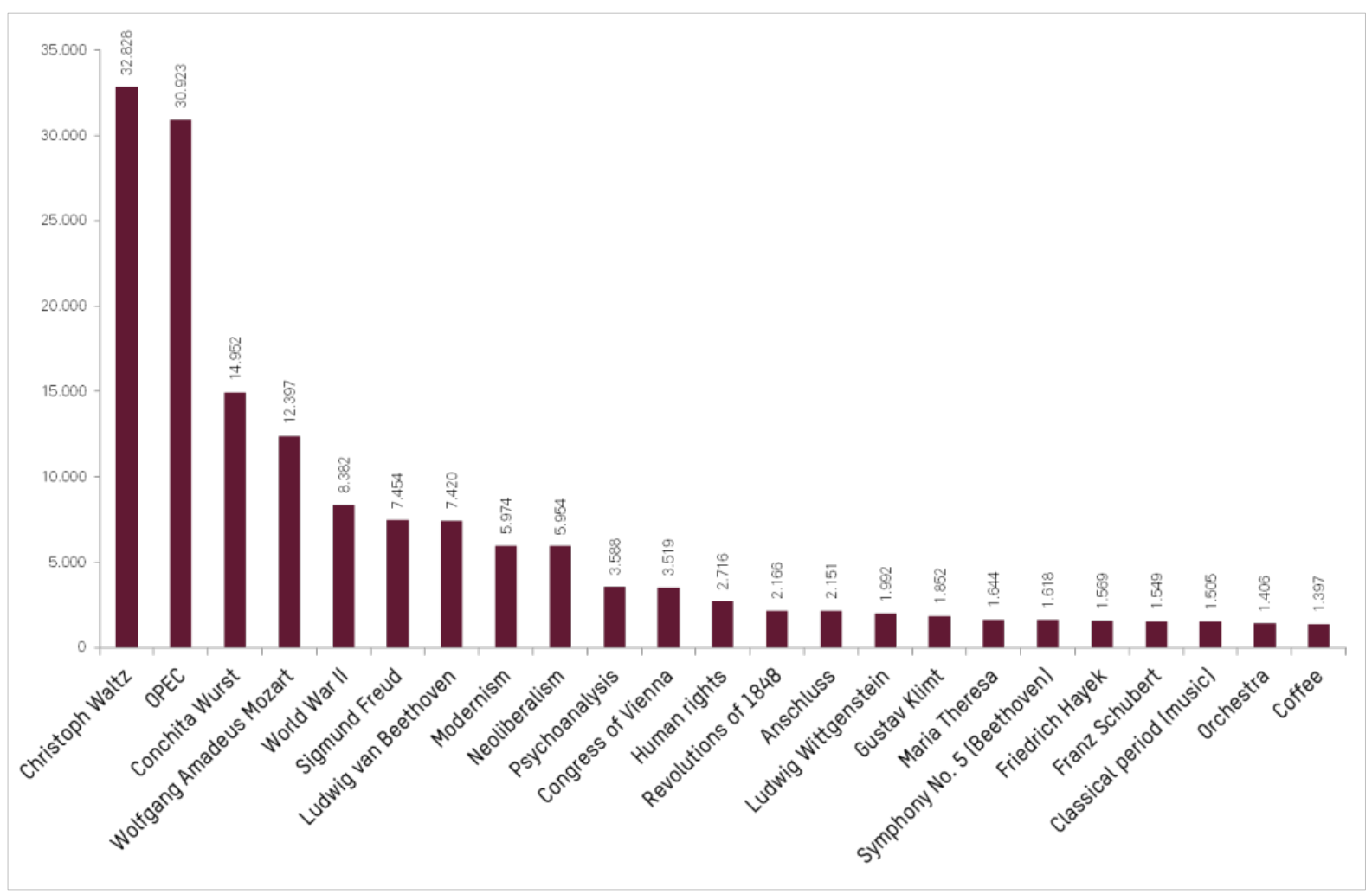

Datenquelle: Wikipedia. $\mathrm{N}=30.216$ Artikel. Analyse: FASresearch

Die explorative Analyse der Kontexte aller englischsprachigen Wikipedia-Artikel, in denen der Begriff "Vienna" eine maßgebliche Bedeutung hat, bestätigt traditionelle Bilder und Mythen der Stadt Wien als Ort des Ursprungs und der Begegnung unterschiedlicher, zum Teil gegensätzlicher wissenschaftlicher, politischer und kultureller Ideen.

Wien erscheint zunächst einmal als ein Ort der Geschichte, und dementsprechend sind es historische Artikel, die zuerst auftauchen: über den Ersten Weltkrieg (Erinnerungsjahr 2014), vor allem aber natürlich über den Zweiten Weltkrieg, den Nationalsozialismus, Adolf Hitler, den Antisemitismus und den Holocaust. Wien zeigt sich auch als einer der Standorte der Vereinten Nationen sowie der OPEC. Darüber hinaus hat Wien 2014 international auf sich aufmerksam gemacht, als Conchita Wurst den Eurovision Song Contest gewann, der ebenfalls in der Liste enthalten ist. Musik spielt eine bedeutende Rolle (Oper, Mozart), Schauspieler (Christoph Waltz), die Kunst (Gustav Klimt), Freud und die Psychoanalyse, und auch politische bzw. ökonomische Theorien (Sozialismus, Neoliberalismus) und Ideen. 
Die meistgelesenen Wikipedia-Artikel mit Wienbezug lassen sich in insgesamt fünf Themenfelder sortieren: 1) der Bereich der Geschichte der ersten Hälfte des 20. Jahrhunderts (erster, zweiter Weltkrieg, Adolf Hitler, Antisemitismus); 2) das Feld Kunst und Kultur (Beethoven, Mozart, Wiener Oper, Sezession, Kaffeehaus), 3) das Feld der Ideengeschichte (Psychoanalyse, Medizin, Wiener Kreis, Austrian School of Economics, das Rote Wien), 4) das Feld der internationalen Politik (UNO-Standort, Stadt der Begegnung zwischen Ost und West) sowie 5) der Bereich bekannter Einzelpersonen (Conchita Wurst, Christoph Waltz, Niki Lauda). Diese fünf Dimensionen prägten im Jahr 2014 das Wienbild in der Welt der Wikipedia, ein Bild, das solcherart als Ort der kulturellen und sozialen Innovationen und Begegnungen, aber weniger als Ort der technologischen Erneuerung definiert ist.

\section{Wissenschaft und Forschung in Wien}

\subsection{Die Wiener Partizipation an der Grundlagenforschung des FWF}

Der Anteil der Wiener Grundlagenforschung an der österreichweiten ist gemessen an den FWF-Projekten in den Jahren 1995 bis 2013 stabil geblieben und beträgt im Durchschnitt $54,5 \%$.

\section{Abb. 6 - Anzahl der FWF-ProJekte, Wlen und österrelchwelt Im Zeltraum 1995 - 2013}

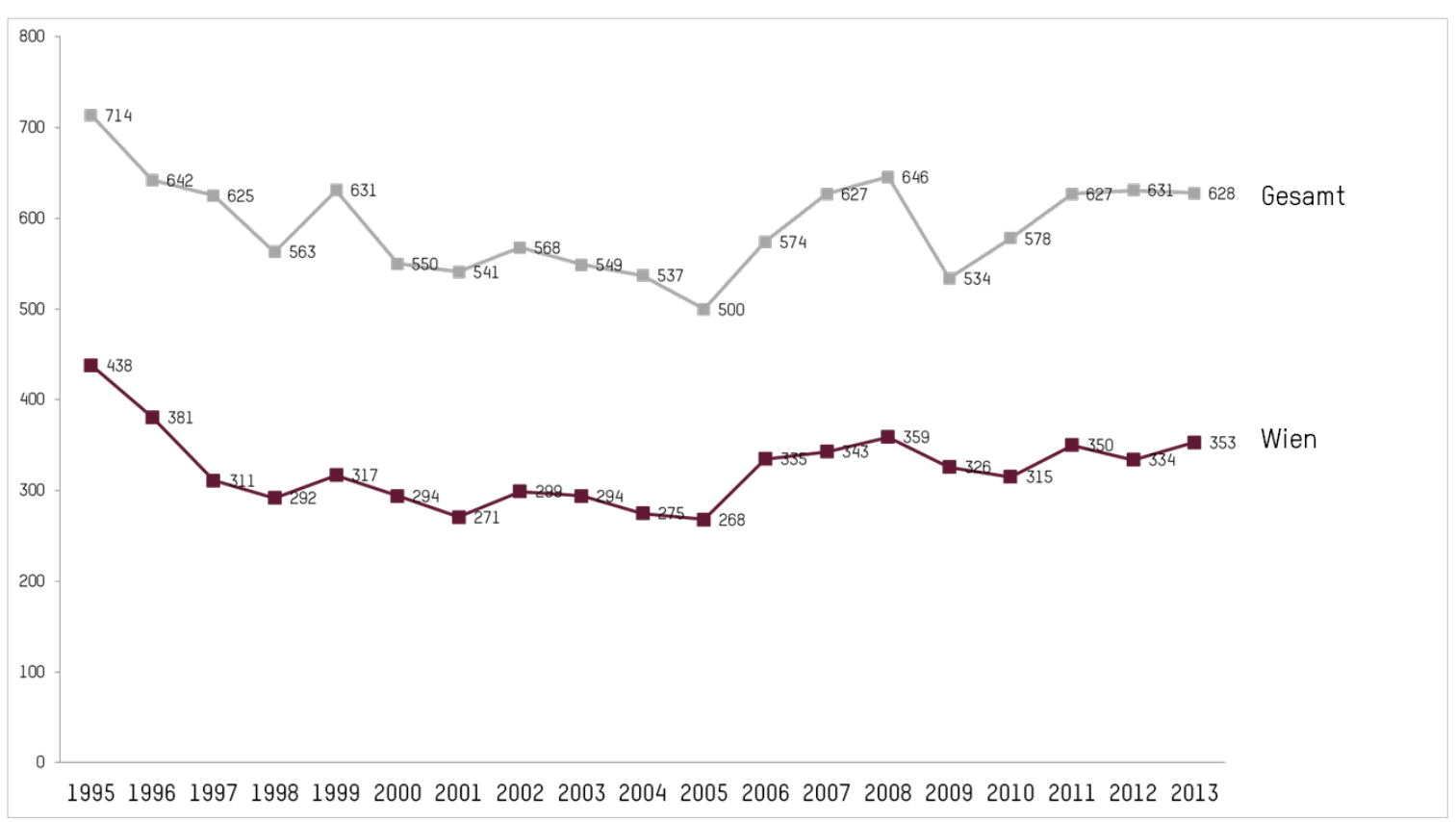


Datenquelle: FWF-Datenbank. N = 11.706 Projekte. Analyse: FASresearch

Der größte Teil der Projekte findet in den Naturwissenschaften statt (41,7\% der Projekte im Zeitraum 2009 bis 2013), gefolgt von den Geisteswissenschaften (25,9\%), der Medizin (13\%), den Sozialwissenschaften (10,8\%), den Technischen Wissenschaften (6,6\%) sowie den Agrarwissenschaften und der Veterinärmedizin (2,1\%). Über die Verteilung der finanziellen Mittel liegen keine öffentlich zugänglichen Daten vor; dass der finanzielle Aufwand in den unterschiedlichen Disziplinen auch unterschiedlich hoch ist, muss bei dieser Aufstellung berücksichtigt werden. Dass der Anteil der technischen und Ingenieurwissenschaften an der FWF-finanzierten Grundlagenforschung insgesamt relativ gering ist, wird immer wieder festgestellt, und auch die uns vorliegenden Zahlen bestätigen es.

Innerhalb der Naturwissenschaften ist die Biologie die dominierende Disziplin (27,9\% der Projekte), bei den Geisteswissenschaften ist es der Bereich der Geschichte und Archäologie (16,6\%). Die folgende Tabelle listet die weiteren Disziplinen (3-Steller-Ebene der OEFOS 2012) nach der Anzahl der Projekte in den Jahren 2009 bis 2013 auf:

Tabelle 4 - Wissenschaftsdisziplinen nach der Anzahl der Projekte mit Wiener Betelligung 2009-2013

\begin{tabular}{|c|c|c|c|c|c|c|c|}
\hline Disziplin & 2009 & 2010 & 2011 & 2012 & 2013 & Total & Momentum \\
\hline Biologie & 101 & 87 & 103 & 99 & 104 & 494 & 1,80 \\
\hline Geschichte, Archäologie & 59 & 48 & 54 & 62 & 70 & 293 & 3,60 \\
\hline Physik, Astronomie & 51 & 50 & 52 & 45 & 43 & 241 & $-2,10$ \\
\hline Medizinisch-theoretische Wissenschaften, Pharmazie & 41 & 38 & 44 & 44 & 48 & 215 & 2,00 \\
\hline Mathematik & 38 & 30 & 42 & 42 & 45 & 197 & 2,60 \\
\hline Sprach- und Literaturwissenschaften & 31 & 39 & 40 & 31 & 38 & 179 & 0,60 \\
\hline Informatik & 30 & 36 & 36 & 32 & 34 & 168 & 0,40 \\
\hline Andere Geisteswissenschaften & 26 & 17 & 29 & 29 & 27 & 128 & 1,40 \\
\hline Klinische Medizin & 20 & 22 & 36 & 24 & 24 & 126 & 1,00 \\
\hline Philosophie, Ethik, Religion & 22 & 27 & 30 & 20 & 26 & 125 & 0,10 \\
\hline Kunstwissenschaften & 20 & 23 & 26 & 22 & 28 & 119 & 1,50 \\
\hline Chemie & 20 & 25 & 28 & 23 & 19 & 115 & $-0,40$ \\
\hline Soziologie & 30 & 23 & 18 & 22 & 22 & 115 & $-1,70$ \\
\hline Geowissenschaften & 22 & 19 & 14 & 17 & 22 & 94 & $-0,20$ \\
\hline Elektrotechnik, Elektronik, Informationstechnik & 12 & 10 & 12 & 9 & 11 & 54 & $-0,30$ \\
\hline Gesundheitswissenschaften & 10 & 8 & 8 & 12 & 14 & 52 & 1,20 \\
\hline Andere Naturwissenschaften & 7 & 9 & 10 & 10 & 12 & 48 & 1,10 \\
\hline Rechtswissenschaften & 12 & 7 & 10 & 9 & 9 & 47 & $-0,40$ \\
\hline Wirtschaftswissenschaften & 8 & 4 & 10 & 6 & 13 & 41 & 1,20 \\
\hline Nanotechnologie & 8 & 9 & 8 & 6 & 8 & 39 & $-0,30$ \\
\hline Andere Technische Wissenschaften & 7 & 8 & 6 & 5 & 8 & 34 & $-0,10$ \\
\hline
\end{tabular}




\begin{tabular}{|c|c|c|c|c|c|c|c|}
\hline Land- und Forstwirtschaft, Fischerei & 6 & 6 & 7 & 5 & 9 & 33 & 0,50 \\
\hline Politikwissenschaften & 4 & 3 & 6 & 11 & 7 & 31 & 1,40 \\
\hline Bauwesen & 10 & 8 & 2 & 8 & 3 & 31 & $-1,40$ \\
\hline Andere Sozialwissenschaften & 3 & 6 & 6 & 5 & 10 & 30 & 1,30 \\
\hline Medien- und Kommunikationswissenschaften & 6 & 4 & 9 & 4 & 6 & 29 & 0,00 \\
\hline Psychologie & 2 & 4 & 8 & 7 & 6 & 27 & 1,10 \\
\hline Veterinärmedizin & 4 & 4 & 10 & 4 & 5 & 27 & 0,20 \\
\hline Umweltingenieurwesen, Angewandte Geowissen. & 4 & 3 & 2 & 5 & 9 & 23 & 1,20 \\
\hline $\begin{array}{l}\text { Humangeographie, Regionale Geographie, Raumpla- } \\
\text { nung }\end{array}$ & 5 & 5 & 6 & 3 & 4 & 23 & $-0,40$ \\
\hline Andere Humanmedizin, Gesundheitswissenschaften & 2 & 4 & 6 & 2 & 3 & 17 & 0,00 \\
\hline Medizinische Biotechnologie & 4 & 3 & 3 & 1 & 3 & 14 & $-0,40$ \\
\hline Industrielle Biotechnologie & 1 & 1 & 2 & 4 & 0 & 8 & 0,10 \\
\hline Maschinenbau & 1 & 2 & 1 & 3 & 1 & 8 & 0,10 \\
\hline Werkstofftechnik & 1 & 5 & 0 & 0 & 2 & 8 & $-0,30$ \\
\hline Erziehungswissenschaften & 3 & 2 & 1 & 0 & 2 & 8 & $-0,40$ \\
\hline Chemische Verfahrenstechnik & 2 & 1 & 1 & 1 & 0 & 5 & $-0,40$ \\
\hline Tierzucht, Tierproduktion & 1 & 1 & 0 & 1 & 1 & 4 & 0,00 \\
\hline Medizintechnik & 1 & 1 & 0 & 1 & 1 & 4 & 0,00 \\
\hline Agrarbiotechnologie, Lebensmittelbiotechnologie & 0 & 3 & 0 & 0 & 0 & 3 & $-0,30$ \\
\hline
\end{tabular}

Datenquelle: FWF-Datenbank. Analyse: FASresearch

Die Tabelle enthält auch Kennzahlen für das „Momentum”, in dem sich die Disziplinen gemessen an der Entwicklung der Projektzahl 2009 bis 2013 befinden. Unter Momentum wird hier und in der Folge die Steigung der Trendlinie, die auf Basis einer linearen Regression mit den beobachteten Werten (hier die Anzahl der Projekte pro Jahr) erstellt wird, verstanden. Ein positiver Wert weist insgesamt auf eine zunehmende Anzahl („positives Momentum"), ein negativer Wert auf eine abnehmende Anzahl an Projekten („negatives Momentum") im Zeitraum von 2009 bis 2013 hin. Absolut gesehen ist der Bereich mit der größten Steigerung die Geschichte (+3,6 Projekte), gefolgt von der Mathematik $(+2,6)$, den medizinisch-theoretischen Wissenschaften $(+2)$, der Biologie $(+1,8)$ und den Kunstwissenschaften $(+1,5)$. Fallende Projektzahlen weisen hingegen die Physik $(-2,1)$, die Soziologie $(-1,7)$ und das Bauwesen auf $(-1,4)$.

Vergleicht man den Anteil der Disziplinen an den Wiener Projekten mit den österreichweiten, zeigt sich, dass Wien gerade in den Geistes- und Sozialwissenschaften besonders stark vertreten ist. Projekte im Bereich Geschichte stellen 9\% der Wiener Projekte, aber nur 4,2\% der Projekte ohne Wiener Beteiligung, was eine Differenz von 4,8 Prozentpunk- 
ten ergibt. Die weiteren in Wien überdurchschnittlich vorhandenen Felder sind Sprachund Literaturwissenschaften (1,9 Prozentpunkte Differenz), Andere Geisteswissenschaften $(1,7)$, Philosophie sowie Soziologie (jeweils 1,1 ).

Worauf es nun aber besonders ankommt, ist die Struktur des Wissensnetzwerks, das aus diesen Aktivitäten entsteht (Abb. 7). 
Abb. 7 - Netzwerk der Wissenschaftsdisziplinen der Wiener FWF-Projekte 1995 - Oktober 2014

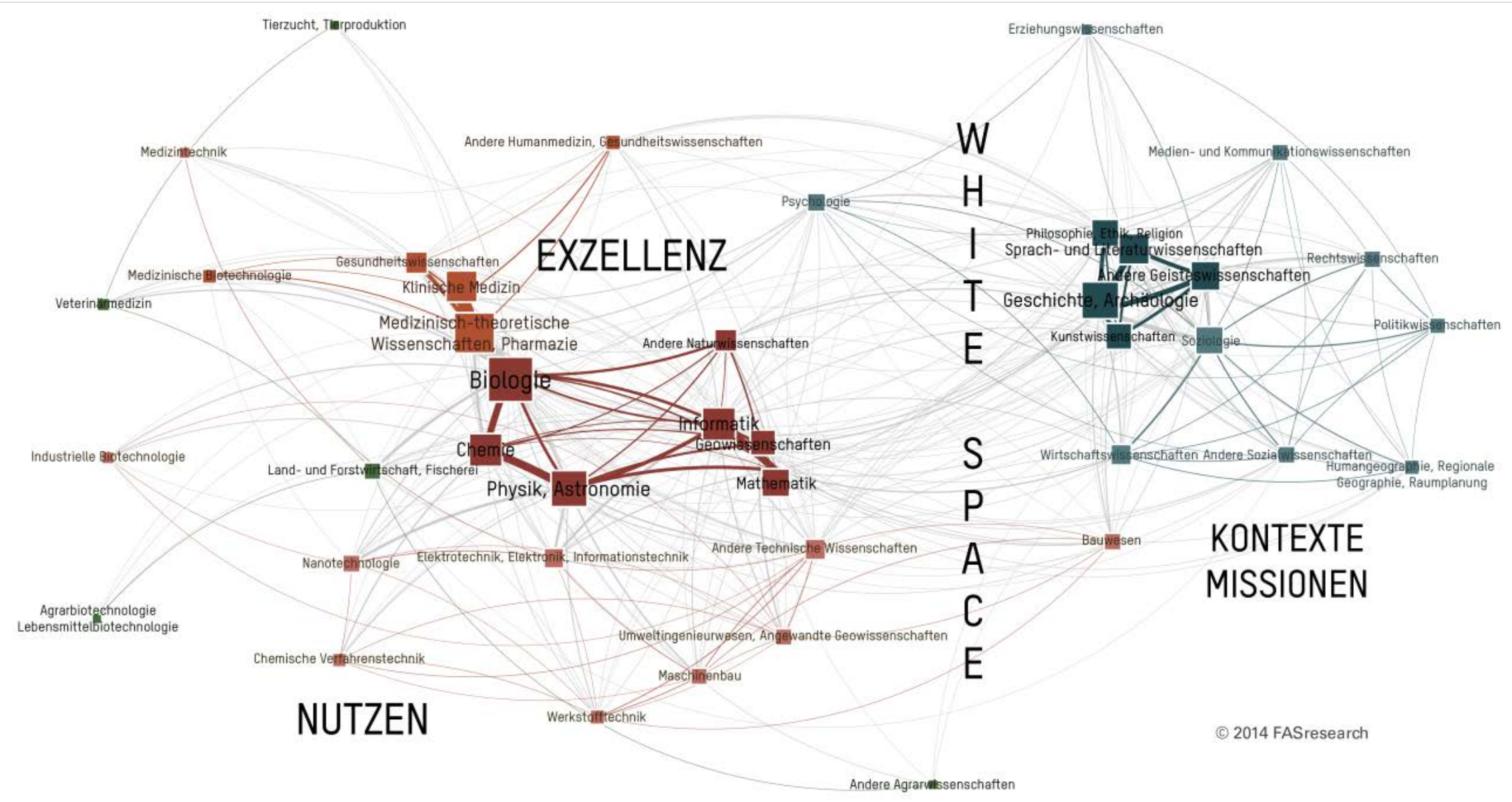

Datenquelle: FWF-Datenbank. N = 6.385 Projekte. Analyse und Grafik: FASresearch. Die Netzwerkknoten stellen Wissenschaftsdisziplinen dar, die FWF-Projekten zugeordnet sind. Einem Projekt können mehrere Disziplinen zugeordnet sein, woraus sich die Verbindungen ergeben (zwei Disziplinen sind dann miteinander verbunden, wenn sie mindestens drei gemeinsamen Projekten zugeordnet wurden). Das Netzwerk ist erkennbar in zwei Hälften geteilt: Naturwissenschaften und Humanmedizin auf der linken, Geis- 


\section{FAS research}

tes- und Sozialwissenschaften auf der rechten Seite. Besonders interessant sind die Verbinder zwischen den Hälften (in der Mitte u.a.: Psychologie, Informatik, Geowissenschaften, Mathematik). Die Größe der Disziplinen ergibt sich aus der Summe der Verbindungen zu anderen Disziplinen. 
Einem FWF-Projekt können von der Antragstellerin/dem Antragsteller mehrere Wissenschaftsdisziplinen zugeordnet werden, um die Forschungsfelder zu kennzeichnen, in denen das Projekt stattfinden soll. Die Abbildung oben veranschaulicht, welche Wissenschaftsdisziplinen besonders häufig durch FWF-finanzierte Projekte verbunden sind, oder anders gesagt, welche einander im Hinblick auf die Art der Forschung ähneln und zwischen welchen mit größerer Wahrscheinlichkeit ein Wissenstransfer stattfindet.

Es zeigt sich die für die Grundlagenforschung typische Teilung in „zwei Kulturen”, die Naturwissenschaften und Humanmedizin einerseits (linke Hälfte) und die Geistes- und Sozialwissenschaften andererseits (rechte Hälfte). Diese beiden Felder sind in sich stärker vernetzt als miteinander. Besonders aufschlussreich ist, durch welche Disziplinen die Verbindungen hergestellt werden und welche damit als Übersetzer zwischen den beiden Kulturen auftreten, nämlich die formalen Wissenschaften (Mathematik und Informatik), weiters die Geowissenschaften sowie auch die Psychologie. Diese Wissenschaften entwickeln Methoden und Modelle, die in beiden Hälften zur Anwendung gelangen (Methoden zur Datensammlung, zur Quantifizierung, statistischen Auswertung sowie zur visuellen Ergebnisdarstellung). Deshalb haben sie das Potential, die beiden Kulturen auf die Gemeinsamkeiten aufmerksam zu machen, die zwischen ihren Fragestellungen und den Antworten, die sie finden, bestehen könnten.

Eine Förderung dieser Übersetzer-Disziplinen könnte dazu geeignet sein, Natur- und Kulturwissenschaften sowie Grundlagenforschung und Anwendung stärker zu integrieren; nicht zuletzt deshalb sind Programme in der Art von "Mathematik und..." so wichtig. Die Teilung ist auch mit einer Kluft zwischen exzellenzorientierten Disziplinen (Naturwissenschaften, linke Hälfte) und solchen, die sich mit dem historischen, gesellschaftlichen und kulturellen Kontext beschäftigen und an interpretierenden, sinngebenden Narrativen arbeiten (rechte Hälfte), verbunden. In der Trennung von Natur- und Kulturwissenschaften sehen wir auch eine Dimension des „White Spaces", d.h. des Potentials, das sich aus fehlenden Beziehungen ergibt. Die andere Dimension des White Spaces in der Grundlagenforschung betrifft den Umstand, dass sich die technischen und Ingenieurwissenschaften 
ganz an der Peripherie der FWF-induzierten Grundlagenforschung befinden (in der Karte sind sie unterhalb der Naturwissenschaften angesiedelt). Vor zehn bis fünfzehn Jahren waren sie noch stärker vertreten, haben dann aber in der FWF-Forschungslandschaft eine immer geringere Rolle gespielt. ${ }^{21}$ Die technischen und Ingenieurwissenschaften vertreten besonders die Nutzensphäre in der Grundlagenforschung, und ihr stärkerer Anschluss an die exzellenz- und an die missions-/kontextorientierte Sphäre wäre im Sinne unseres Innovationsmodells zu begrüßen.

Die FWF-geförderte Grundlagenforschung wird erwartungsgemäß von einigen großen Playern dominiert. Insgesamt führten die Universitäten 77,9\% der Projekte durch (1.379 von 1.771 im Zeitraum 2009 bis 2013), die außeruniversitäre Forschung 18,6\%, Kunst und Kultureinrichtungen (z.B. die großen Museen) 1,7\%. Die Zivilgesellschaft (gemeinnützige Vereine) ist an 11 Projekten beteiligt $(0,6 \%)$, und auch Unternehmen sind so gut wie nicht vorhanden (4 Projekte).

Tabelle 5 - Institutionen (Organisationsebene) nach Anzahl der FWF-Projekte 2009 - 2013

\begin{tabular}{lccc} 
Institution & Projekte & Prozent & Momentum \\
\hline UNIVERSITÄT WIEN & 636 & 35,9 & 2,80 \\
TECHNISCHE UNIVERSITÄT WIEN & 281 & 15,9 & 2,90 \\
MEDIZINISCHE UNIVERSITÄT WIEN & 213 & 12,0 & 0,90 \\
ÖSTERREICHISCHE AKADEMIE DER WISSENSCHAFTEN & 149 & 8,4 & 3,70 \\
UNIVERSITÄT FÜR BODENKULTUR WIEN & 126 & 7,1 & $-0,50$ \\
VETERINÄRMEDIZINISCHE UNIVERSITÄT WIEN & 60 & 3,4 & 0,00 \\
WIRTSCHAFTSUNIVERSITÄT WIEN & 22 & 1,2 & 0,10 \\
AIT AUSTRIAN INSTITUTE OF TECHNOLOGY GMBH & 20 & 1,1 & 0,10 \\
UNIVERSITÄT FÜR ANGEWANDTE KUNST WIEN & 19 & 1,1 & 1,20 \\
IMP-INSTITUT FÜR MOLEKULARE PATHOLOGIE & 17 & 1,0 & 0,10
\end{tabular}

Datenquelle: FWF-Datenbank. Analyse: FASresearch.

Allein die Universität Wien kommt auf mehr als ein Drittel der Projekte. Stark steigende Projektzahlen haben vor allem die Akademie der Wissenschaften, die TU Wien sowie die Universität Wien zu verzeichnen.

${ }^{21}$ FASresearch et al. (Hrsg.): Netzwerke der Wissensproduktion. Wien 2008. 
Auf der Ebene der Fakultäten bzw. Institute ist es die Fakultät für Mathematik der Universität Wien, die das Feld anführt (53 Projekte oder 2,9\%), gefolgt vom Zentrum für Pathophysiologie der MedUni Wien (1,7\%), dem Atominstitut der TU Wien (1,6\%), der Uniklinik für Innere Medizin I sowie dem Institut für Germanistik der Universität Wien.

Tabelle 6 - Instltutionen (Instltutsebene) nach Anzahl der FWF-ProJekte 2009 - 2013

\begin{tabular}{|c|c|c|c|}
\hline Institution & Projekte & Prozent & Momentum \\
\hline UNI WIEN/FAKULTÄT FÜR MATHEMATIK & 53 & 2,9 & 0,5 \\
\hline MEDUNI WIEN/ZENTRUM FÜR PATHOPHYSIOLOGIE & 30 & 1,7 & $-1,4$ \\
\hline TU WIEN/ATOMINSTITUT & 29 & 1,6 & $-0,9$ \\
\hline MEDUNI WIEN/UNIVERSITÄTSKLINIK FÜR INNERE MEDIZIN I & 28 & 1,5 & 2,2 \\
\hline UNI WIEN/INSTITUT FÜR GERMANISTIK & 27 & 1,5 & 0,0 \\
\hline UNI WIEN/FAKULTÄT FÜR PHYSIK & 25 & 1,4 & 0,4 \\
\hline BOKU/DEPARTMENT FÜR CHEMIE & 25 & 1,4 & $-0,2$ \\
\hline TU WIEN/INSTITUT FÜR VERFAHRENSTECHNIK, UMWELTTECHNIK... & 22 & 1,2 & 0,9 \\
\hline TU WIEN/INSTITUT FÜR DISKRETE MATHEMATIK UND GEOMETRIE & 21 & 1,2 & 0,0 \\
\hline UNI WIEN/INSTITUT FÜR GESCHICHTE & 21 & 1,2 & $-2,2$ \\
\hline TU WIEN/INSTITUT FÜR THEORETISCHE PHYSIK & 20 & 1,1 & 0,1 \\
\hline MEDUNI WIEN/DEPARTMENT FÜR MEDIZINISCHE BIOCHEMIE & 19 & 1,0 & $-0,3$ \\
\hline ÖAW/INSTITUT FÜR MITTELALTERFORSCHUNG & 18 & 1,0 & 0,6 \\
\hline ÖAW/INSTITUT FÜR KULTURGESCHICHTE DER ANTIKE & 18 & 1,0 & 0,5 \\
\hline TU WIEN/INSTITUT FÜR INFORMATIONSSYSTEME & 17 & 0,9 & 1,9 \\
\hline UNI WIEN/DEPARTMENT FÜR MIKROBIOLOGIE... & 17 & 0,9 & 0,5 \\
\hline UNI WIEN/INSTITUT FÜR KUNSTGESCHICHTE & 17 & 0,9 & 0,4 \\
\hline UNI WIEN/KURT GÖDEL CENTER FOR MATHEMATICAL LOGIC & 17 & 0,9 & 0,0 \\
\hline BOKU/DEPARTMENT FÜR ANGEWANDTE GENETIK & 16 & 0,9 & 1,5 \\
\hline CEMM-FORSCHUNGSZENTRUM FÜR MOLEKULARE MEDIZIN GMBH & 16 & 0,9 & 0,7 \\
\hline IMP-INSTITUT FÜR MOLEKULARE PATHOLOGIE & 16 & 0,9 & 0,0 \\
\hline UNI WIEN/INSTITUT FÜR PHILOSOPHIE & 15 & 0,8 & 0,3 \\
\hline IMBA-INSTITUT FÜR MOLEKULARE BIOTECHNOLOGIE & 15 & 0,8 & $-0,2$ \\
\hline UNI WIEN/INSTITUT FÜR ALTE GESCHICHTE & 14 & 0,8 & 1,0 \\
\hline AIT/DEPARTMENT OF HEALTH AND ENVIRONMENT & 14 & 0,8 & 0,5 \\
\hline
\end{tabular}

Datenquelle: FWF-Datenbank. Analyse: FASresearch.

Die TU Wien tritt als besonders starker Player in den oben angeführten Übersetzer-

Wissenschaften auf (Mathematik, Informatik), sowie in der Physik, aber eben nicht, soweit es den FWF betrifft, in den technischen und Ingenieurwissenschaften - so erklärt sich die Auffälligkeit des geringen Anteils der technischen Wissenschaften und dem hohen der TU Wien an den FWF-Projekten. Auch die Uni Wien ist stark in den formalen Wissenschaften vertreten, abgesehen davon, dass sie ohnehin unter ihrem Dach die unterschiedlichen 
Kulturen der Grundlagenforschung versammelt (Biologie, Physik, Geschichte, Sprachen und Philosophie). Die Schwerpunkte der MedUni Wien bilden natürlich die medizinische Grundlagenforschung („medizinisch-theoretische Wissenschaften“), die klinische Medizin, die Biologie und die Gesundheitswissenschaften. Die Akademie der Wissenschaften ist vor allem in den Bereichen Geschichte/Archäologie sowie in den Sprach- und Literaturwissenschaften vertreten, die BOKU in der Biologie, in der Land- und Forstwirtschaft, in der Chemie sowie in den Geowissenschaften, womit sie ebenfalls zu den Institutionen gehört, die in den Übersetzer-Disziplinen eine große Rolle spielen. Letzteres trifft, was den FWF angeht, nicht auf das AIT zu, das ansonsten, wie wir noch sehen werden, ein sehr breites Innovationsfeld abdeckt, sich beim FWF aber zumindest im Hinblick auf die letzten Jahre auf Biologie, Physik und Chemie konzentriert.

Zusammenfassend stellen wir für die FWF-geförderte Wiener Grundlagenforschung fest:

- Einen stabilen Anteil an den FWF-Projekten über den Untersuchungszeitraum.

- Stärkefelder sind die Biologie, die Geschichte, die Physik, Life Sciences und die Mathematik.

- Von diesen verzeichnen vor allem die Geschichte, die Mathematik und die Medizin ein positives Momentum.

- Typische Teilung in Natur- sowie Geistes- bzw. Sozialwissenschaften.

- Wien ist stark vertreten in den Übersetzerdisziplinen (Mathematik, Informatik, Geowissenschaften, Psychologie).

- Unterdurchschnittliches Vorhandensein der technischen und Ingenieurwissenschaften.

\subsection{Wissenschaft und Forschung im WWTF}

Der Wiener Wissenschafts-, Forschungs- und Technologiefonds (WWTF) zielt auf die Förderung der Spitzenforschung sowie der Stärkefelder Wiens ab, was sich auch in den Anteilen zeigt, die die verschiedenen Förderprogramme an Gesamtausschüttungen 2009 bis 
2013 (insgesamt über 61,8 Mio. Euro laut Forschungsdatenbank des WWTF) haben. Life Sciences und IKT führen die Liste an (37,4\% bzw. 21,2\%); diesen zwei Schwerpunkten werden wir auch noch in einem anderen Kontext begegnen, nämlich bei der Patenlandkarte Wiens. Es folgt das Programm "Mathematik und..." (13,4\%), dessen Bedeutung für die Stärkung der Beziehungen zwischen den Kulturen der Grundlagenforschung schon im Zusammenhang mit dem FWF-Netzwerk hervorgehoben wurde. Auch den Geistes-, Sozial- und Kulturwissenschaften sind eigene Programme gewidmet (10,8\%), weiters einem Universitäts-Infrastruktur-Programm (9,9\%) und den Kognitionswissenschaften (7,3\%), die ebenfalls zu den Übersetzer-Disziplinen gehören, insbesondere, was die Verbindung zwischen Natur- und Sozialwissenschaften betrifft.

Abb. 8 - Prozentantell der WWTF-Programme an den Gesamtaufwendungen 2009 - 2013

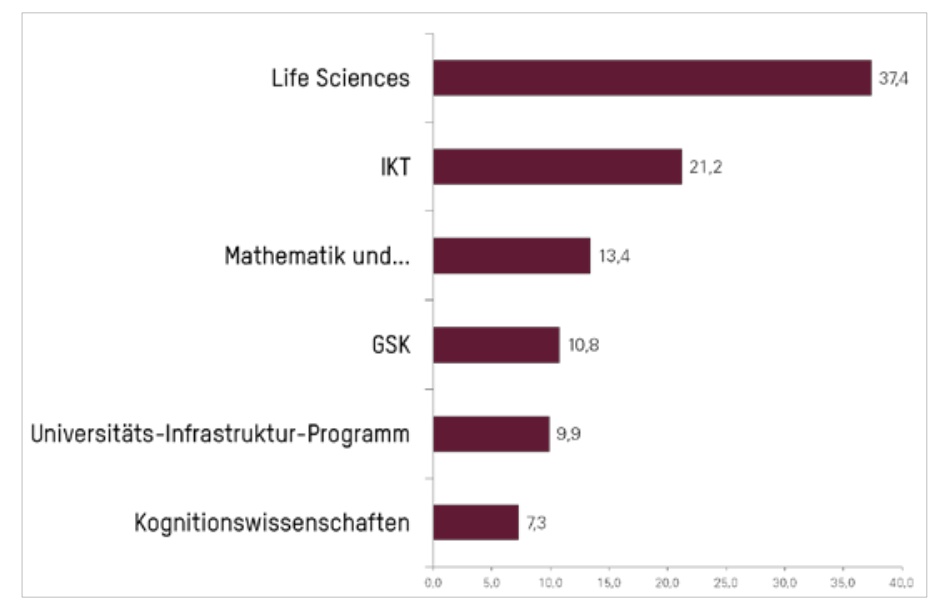

Datenquelle: WWTF-Datenbank. $N=258$ Projekte. Analyse: FASresearch.

Das Momentum beim WWTF hängt in hohem Maße davon ab, in welchen Jahren es welche Schwerpunktsetzungen bei den Förderprogrammen gibt. Was die Adressaten betrifft, so gibt es eine große Schnittmenge zwischen den Institutionen, die der WWTF fördert, und jenen, die beim FWF vorkommen. 75\% der Projekte zwischen 2009 und 2013 wurden von Universitäten durchgeführt, 15\% von der außeruniversitären Forschung; Unternehmen und sonstige Institutionen spielen beim WWTF (wie beim FWF) nur eine geringe Rolle.

Die Institutionen mit den meisten WWTF-Projekten 2009 bis 2013 sind:

Tabelle 7 - Instltutlonen nach der Zahl der WWTF-Projekte 2009 bls 2013 


\begin{tabular}{lccc} 
Institution & Gesamt & Prozent & Momentum \\
\hline UNIVERSITÄT WIEN & 46 & 23 & $-0,5$ \\
TECHNISCHE UNIVERSITÄT WIEN & 33 & 16,5 & 0,2 \\
MEDIZINISCHE UNIVERSITÄT WIEN & 25 & 12,5 & 0,1 \\
UNIVERSITÄT FÜR BODENKULTUR WIEN & 11 & 5,5 & $-0,2$ \\
AKADEMIE DER BILDENDEN KÜNSTE WIEN & 10 & 5 & 0,2 \\
VETERINÄRMEDIZINISCHE UNIVERSITÄT WIEN & 10 & 5 & $-0,1$ \\
AIT AUSTRIAN INSTITUTE OF TECHNOLOGY GMBH & 7 & 3,5 & $-0,4$ \\
ÖSTERREICHISCHE AKADEMIE DER WISSENSCHAFTEN & 7 & 3,5 & $-0,4$ \\
UNIVERSITÄT FÜR ANGEWANDTE KUNST WIEN & 7 & 3,5 & $-0,8$ \\
WIRTSCHAFTSUNIVERSITÄT WIEN & 6 & 3 & $-0,1$
\end{tabular}

Datenquelle: WWTF-Datenbank. $\mathrm{N}=258$ Projekte. Analyse: FASresearch. 
Abb. 9 - Beteiligung von Institutionen an WWTF-Förderprogrammen 2009 - 2013 (Ausschnitt)

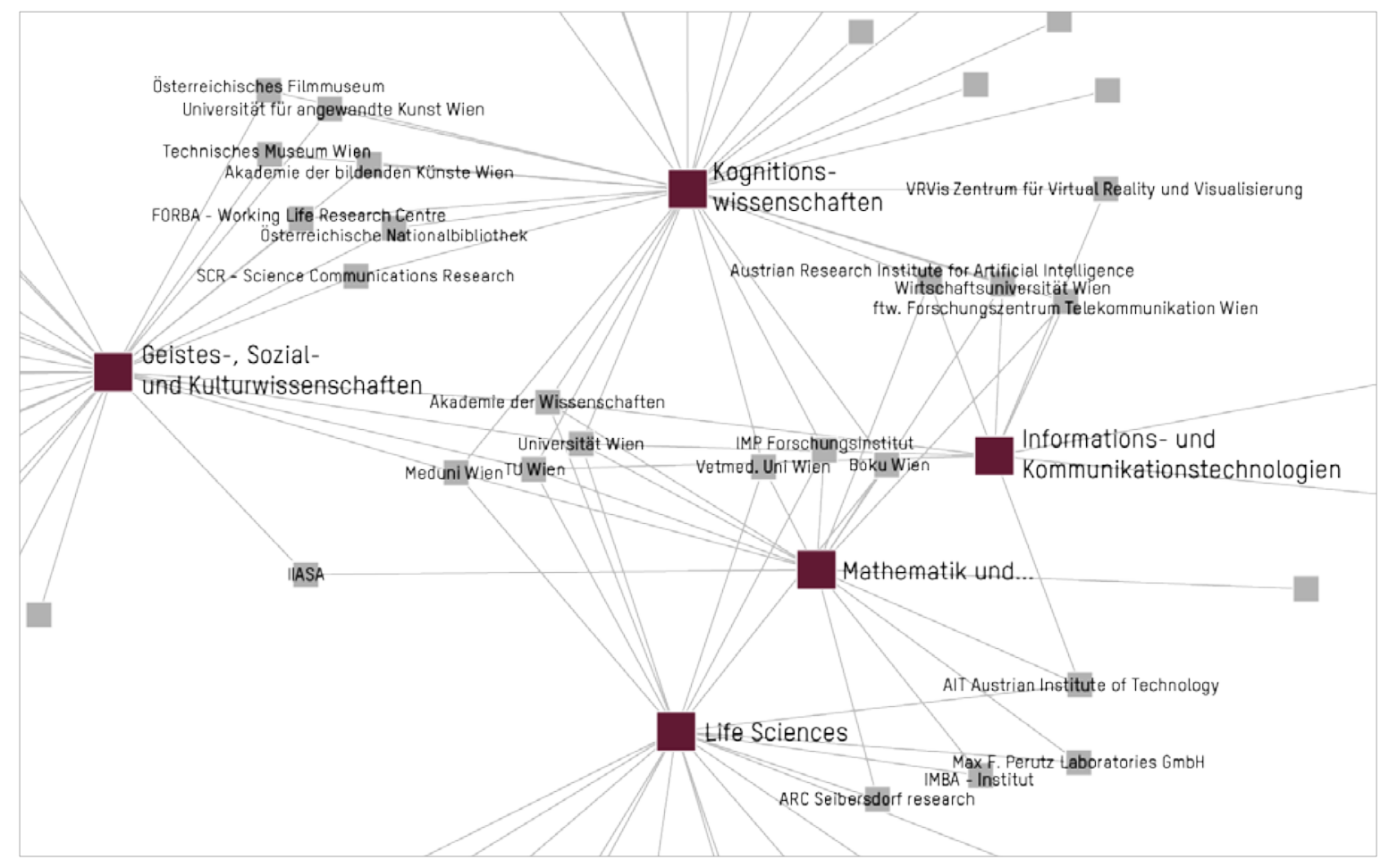

Datenquelle: WWTF-Datenbank. N = 258 Projekte. Analyse und Grafik: FASresearch. Die grauen Netzwerknoten stellen die Institutionen dar, die roten die WWTF-Förderprogramme. Eine Verbindung besteht dann, wenn eine Institution mindestens einmal am entsprechenden Förderprogramm teilgenommen hat.

Die Abbildung oben zeigt, welche Institutionen sich an welchen Förderprogrammen beteiligen (ohne Rücksicht auf das Volumen der Partizipation). Interessant aus der Sicht unseres Innovationsmodells sind die Akteure, die die unterschiedlichen Kulturen (GSK einerseits, Life Sciences, formale Wissenschaften sowie IKT andererseits) verbinden. Sie sind in der Mitte der Abbildung angesiedelt, und es handelt sich um die großen Player Uni Wien, TU, MedUni und ÖAW. Links oben finden wir jene Akteure, die sich auf die GSK und die Kognitionswissenschaften konzentriert haben (Universität für angewandte Kunst, Akademie der bildenden Künste, Nationalbibliothek, Technisches Museum, Filmmuseum, FORBA, SCR), links unten die der Life Sciences, IKT und Mathematik (z.B. das AIT).

Zusammenfassend zum WWTF:

- Der WWTF unterstützt die Grundlagenforschung in Wien und fördert dabei zusätzlich die Stärkefelder, die es in Wien gibt. 
- Zu diesen gehören vom Projektvolumen her vor allem die Life Sciences und die IKT, denen wir auch im Zusammenhang mit der Patentlandschaft als Schwerpunkte begegnen werden.

- Die WWTF-induzierte Forschung wird hauptsächlich von den Universitäten durchgeführt, zu einem geringeren Teil auch von der außeruniversitären Forschung. Vor dem Hintergrund der finanziellen Lage der Universitäten sind Instrumente wie der WWTF ebenso wie der FWF unabdingbar für die dort stattfindende Forschung.

- Unternehmen sowie die Zivilgesellschaft kommen in der Sphäre des WWTF hingegen nicht oder so gut wie gar nicht vor. Nun erscheint die Einbindung der Wirtschaft nicht als die oberste Priorität des WWTF, da es dafür andere, gut funktionierende Förderinstrumente gibt. Was die Verbindung der Sphären der wissenschaftlichen Exzellenz einerseits und der Mission bzw. der Lösung gesellschaftlicher Probleme andererseits betrifft, könnte der WWTF aus unserer Sicht durch ein Mehr an Partizipation der Zivilgesellschaft eine stärkere Rolle als Übersetzer spielen. Anknüpfungspunkte könnten sich aus den Fragestellungen der Programme ergeben, die in vielen Fällen gesellschaftliche Problemlagen oder Fragestellungen betreffen (z.B. Thema öffentlicher Raum bei den GSK-Programmen, Diversität - Identität, IKT, Life Sciences etc.). Der Aspekt der Lösung gesellschaftlicher Probleme wird auch auf EU-Ebene (Horizon2020: „Societal Challenges”) eine größere Rolle spielen.

\subsection{Publikationen in den Bereichen der Life Sciences und Technologie}

Forschungsprojekte sind eine Dimension des Outputs der wissenschaftlichen Sphäre; eine weitere sind die Publikationen und deren Qualität, oder besser gesagt: ihr Impact. Es hätte den Rahmen des Projekts gesprengt, hinreichend Daten zu generieren und zu analysieren, um ein repräsentatives Abbild des gesamten Publikationsoutputs Wiens in den verschiedenen Innovationsbereichen zu bekommen. Aus diesem Grund haben wir uns auf zwei Bereiche konzentriert, die auch in anderen Zusammenhängen (WWTF, Wirtschaftsagentur, Patente) genannt werden, nämlich die Life Sciences und den Bereich der Tech- 
nologie (Elektrotechnik, Elektronik, Computerwissenschaften), und uns auf zwei wichtige Datenquellen in diesen Bereichen beschränkt, nämlich Pubmed und IEEE.

Wenden wir uns zunächst dem Bereich der Life Sciences zu. Die starke Zunahme an Publikationen im Bereich der Humanmedizin wird immer wieder konstatiert, und auch die uns vorliegenden Daten bekräftigen diesen Eindruck.

Abb. 10 - Anzahl der Pubmed-Publikationen mit Wiener Beteiligung 2009 - 2013

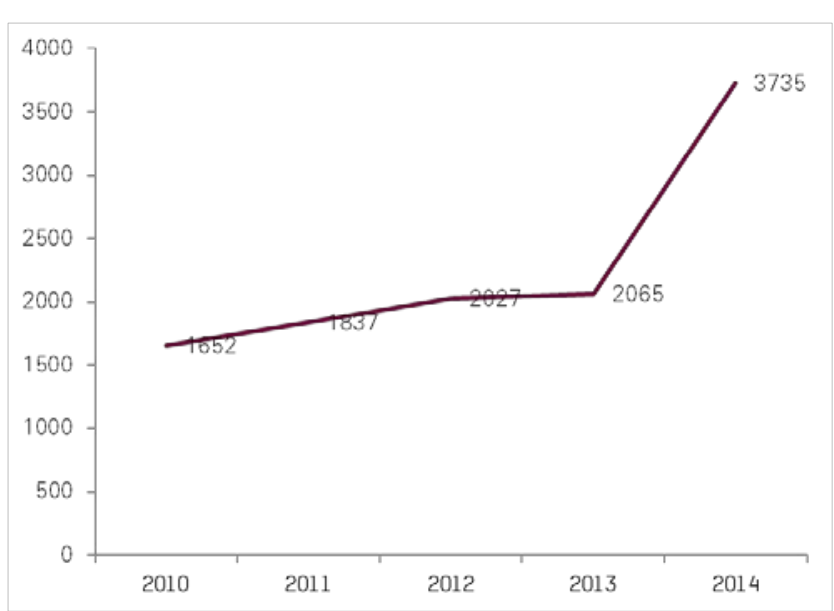

Datenquelle: Pubmed. N = 11.316 Publikationen. Analyse: FASresearch

Die Zahlen spiegeln eine massive Zunahme an Publikationen wider, von 2010 auf 2014 beträgt der Anstieg 126\%. Das ist auch der Grund, warum so gut wie alle SCOPUSKategorien von medizinischen Fachbereichen (162 von 182), mit denen die Journale versehen sind, in denen die Wiener Publikationen erschienen, ein positives Momentum aufweisen.

Am häufigsten sind die Wiener Publikationen in den Bereichen Biochemie (4,7\% aller Zuordnungen), Molekularbiologie (4,5\%), Genetik (3,1\%), Onkologie (2,8\%), Hämatologie $(2,4 \%)$, Zellbiologie $(2,4 \%)$, Chirurgie $(2,3 \%)$, Radiologie $(2,3 \%)$ sowie Immunologie $(2,2 \%)$ angesiedelt. Die stärkste absolute Zunahme an Zuordnungen finden wir in den Bereichen Molekularbiologie, Genetik, Onkologie, Biochemie, Krebsforschung, Kardiologie, Chirurgie sowie Urologie und in der systemischen Biologie/Ökologie (Evolutionsbiologie, Komplexitätsforschung). 
Abb. 11 - Netzwerk der Wlener Publlkatlonen Im Berelch der Llfe Sclences 2010 - 2014

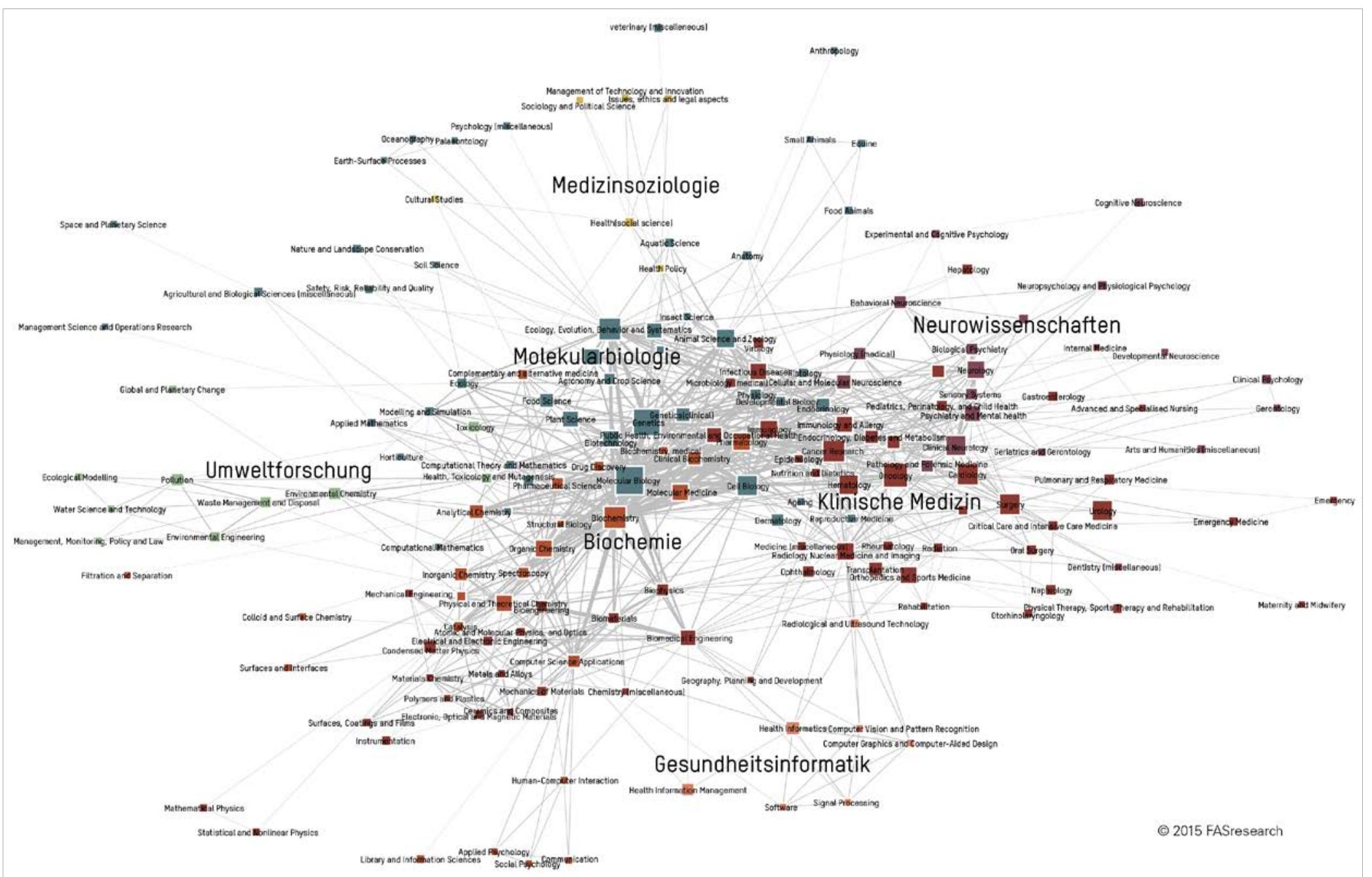

Datenquelle: Pubmed. N = 11.316 Publikationen. Analyse und Grafik: FASresearch. Die Verbindungen zwischen den Disziplinen entstehen dadurch, dass eine Publikation in mehreren Disziplinen gleichzeitig angesiedelt sein kann. Je stärker eine Linie zwischen zwei Disziplinen, desto größer die Anzahl der gemeinsamen Projekte. Je größer eine Disziplin, mit desto mehr anderen Disziplinen ist sie verbunden. 
Die Abb. 11 zeigt, wie die SCOPUS-Kategorien, die die Forschungsbereiche der Medizin bezeichnen, dadurch miteinander durch Wiener Publikationen verbunden sind, dass eine Publikation in zwei oder mehreren Bereichen angesiedelt sein kann. Die Zentren des medizinischen Publikationsoutputs sind deutlich identifizierbar: Medizinische Grundlagenforschung auf der linken Seite (Molekularbiologie, Biochemie) und klinische Forschung auf der rechten (Onkologie, Kardiologie, Chirurgie, Urologie etc.). Rund um diese beiden Zentren finden wir interessante Bereiche, bei denen das relative Momentum (d.h. die prozentuelle Zunahme an Zuordnungen von Klassifikationen) besonders hoch ist: die Neurowissenschaften, die sich im Sektor der klinischen Medizin befinden (deutliche Distanz zur Molekularbiologie), Gesundheitsinformatik, Umweltforschung, Bioengineering und Medizinsoziologie.

Gerade wenn es zutrifft, dass die Life Sciences und die IKT zwei wichtige Stärkefelder Wiens darstellen, dann sind jene Bereiche umso wichtiger, die Verbindungen zwischen den beiden Feldern herstellen können. Dazu zählen vor allem die Gesundheitsinformatik und die Medizintechnologie. Die Medizinsoziologie wiederum würde zwischen dem Feld der wissenschaftlichen Exzellenz und dem der Mission/Problemlösung Übersetzungsarbeit leisten.

Tabelle 8 - Wlener Llfe Sclence Publlkationen 2010 - 2013: dle wichtlgsten SCOPUS-Klassen

\begin{tabular}{lccc} 
SCOPUS-Klassen & Zuordnungen & Prozent & Momentum \\
\hline Biochemistry & 979 & 4,71 & 19,5 \\
Molecular Biology & 936 & 4,50 & 32,9 \\
Genetics & 641 & 3,08 & 30,2 \\
Oncology & 582 & 2,80 & 23,7 \\
Hematology & 498 & 2,39 & 15,0 \\
Cell Biology & 497 & 2,39 & 15,8 \\
Surgery & 496 & 2,38 & 17,3 \\
Radiology Nuclear Medicine and imaging & 478 & 2,30 & 11,5 \\
Cancer Research & 476 & 2,29 & 19,3 \\
Immunology & 457 & 2,20 & 11,8 \\
Cardiology and Cardiovascular Medicine & 448 & 2,15 & 18,2 \\
Ecology, Evolution, Behavior and Systematics & 401 & 1,93 & 19,5 \\
Infectious Diseases & 366 & 1,76 & 10,1 \\
Immunology and Allergy & 360 & 1,73 & 9,4 \\
Biotechnology & 354 & 1,70 & 9,9
\end{tabular}




$\begin{array}{lccc}\text { Molecular Medicine } & 348 & 1,67 & 10,1 \\ \text { Clinical Neurology } & 341 & 1,64 & 15,6 \\ \text { Animal Science and Zoology } & 313 & 1,50 & 13,2 \\ \text { Pharmacology } & 302 & 1,45 & 11,6 \\ \text { Analytical Chemistry } & 286 & 1,37 & 6,5 \\ \text { Organic Chemistry } & 259 & 1,24 & 10,5 \\ \text { Clinical Biochemistry } & 258 & 1,24 & 5,4 \\ \text { Neurology } & 246 & 1,18 & 11,8 \\ \text { Microbiology } & 228 & 1,10 & 9,1 \\ \text { Orthopedics and Sports Medicine } & 224 & 1,08 & 10,4 \\ \text { Physical and Theoretical Chemistry } & 217 & 1,04 & 9,7 \\ \text { Public Health, Environmental Health } & 214 & 1,03 & 8,2 \\ \text { Ophthalmology } & 213 & 1,02 & 3,7 \\ \text { Biomedical Engineering } & 207 & 0,99 & 8,8 \\ \text { Endocrinology, Diabetes and Metabolism } & 202 & 0,97 & 5,8\end{array}$

Datenquelle: Pubmed. N = 11.316 Publikationen. Analyse: FASresearch

Wenden wir uns nun den Publikationen im technologischen Bereich (Elektrotechnik, Elektronik, Computerwissenschaften) zu. Die Schlagworte, mit denen die Wiener Publikationen im Zeitraum 2002 bis 2013 am häufigsten versehen waren, sind:

Tabelle 9 - Dle wichtlgsten Felder Wlener Publlkatlonen Im Technologleberelch (IEE-Datenbank, Schlagworte)

\begin{tabular}{|c|c|c|}
\hline Schlagworte & Publikationen 02-13 & Momentum 09-13 \\
\hline Internet & 191 & $-0,2$ \\
\hline Web services & 179 & $-4,5$ \\
\hline embedded systems & 170 & -4 \\
\hline finite element analysis & 165 & $-1,6$ \\
\hline data visualisation & 141 & 3,4 \\
\hline III-V semiconductors & 136 & $-0,9$ \\
\hline channel estimation & 127 & 0,6 \\
\hline quality of service & 126 & $-0,5$ \\
\hline wireless sensor networks & 121 & 0,9 \\
\hline software architecture & 121 & -4 \\
\hline cloud computing & 119 & 9,1 \\
\hline optimisation & 118 & 0,6 \\
\hline quantum cascade lasers & 117 & $-0,2$ \\
\hline MOSFET & 115 & 0,1 \\
\hline business data processing & 103 & 1 \\
\hline MIMO communication & 103 & $-1,1$ \\
\hline semiconductor device models & 101 & 1,3 \\
\hline silicon & 99 & $-1,6$ \\
\hline security of data & 91 & $-3,1$ \\
\hline protocols & 89 & $-0,3$ \\
\hline
\end{tabular}




$\begin{array}{lcc}\text { gallium arsenide } & 88 & -1,1 \\ \text { formal specification } & 85 & 0,5 \\ \text { Monte Carlo methods } & 82 & 0,3 \\ \text { ontologies (artificial intelligence) } & 81 & -1,5 \\ \text { real-time systems } & 81 & -0,6\end{array}$

Datenquelle: IEEE. N = 5.364 Publikationen. Analyse: FASresearch

Jene Schlagworte, die die größten Zuwächse an Publikationen im Zeitraum von 2009 bis 2013 zu verzeichnen haben, sind: Cloud Computing, Smart Power Grids, Data Visualisation, Quality of Experience (Usability), Power Engineering Computing und Electric Vehicles. Man muss bei der Interpretation der Schlagworte vorsichtig sein, denn nicht immer kennzeichnet ein aufsteigender Begriff ein neues Forschungsfeld, sondern weist vielmehr auf terminologische Änderungen in der Klassifikation hin. Die Tendenzen aber sind klar, die Bereiche mit positivem Momentum finden sich vor allem im Zusammenhang mit Internettechnologien, Datenspeicherung, Datenanalyse und -darstellung sowie intelligenten Stromnetzen. Rund um die thematischen Zentren herum (siehe die folgende Netzwerkkarte) lassen sich weitere spannende, noch ungenügend verbundene Potentialfelder im Umfeld von Datenvisualisierung und Embedded Systems, insbesondere Artificial Intelligence, Decision Making, Semantic Networks und Agenten-basierte Simulation erkennen. 
Abb. 12 - Netzwerk der Wlener Publlkatlonen Im Berelch Technologle 2002 - 2013; Schlagworte, dle durch mIndestens drel Publlkatlonen verbunden sInd

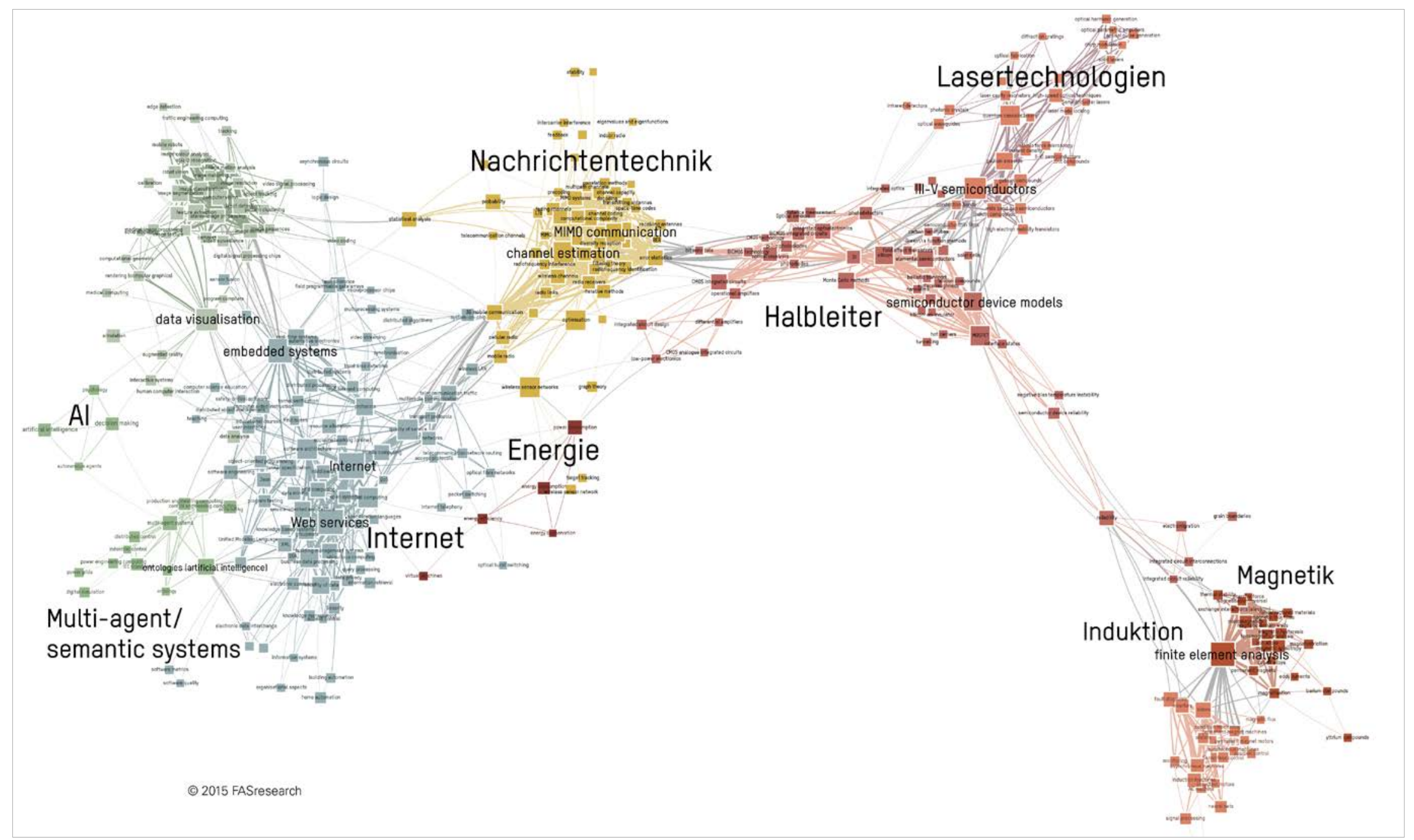




\section{FAS research}

Datenquelle: IEEE. N = 5.364 Publikationen. Analyse und Grafik: FASresearch. Die Verbindungen zwischen den Schlagworten (Disziplinen) entstehen dadurch, dass einer Publikation gleichzeitig mehrere Schlagworte zugeordnet sein können. Je größer ein Schlagwort, mit desto mehr anderen Schlagworten ist es verbunden. 


\subsection{Partizipation an EU-Rahmenprogrammen}

Die EU-Rahmenprogramme betreffen alle drei Währungen der Innovation. Sie fördern Exzellenz- und Grundlagenforschung ebenso wie Anwendungsorientierung und industrienahe Forschung, und darüber hinaus gibt es im jüngsten Rahmenprogramm Horizon2020 auch den Bereich "Societal Challenges", der sich auf die Lösung wirtschaftlicher bzw. sozialer Probleme bezieht und im Rahmen dessen auch die Zivilgesellschaft (CSOs - Civil Society Organisations) stärker eingebunden werden soll. Die Partizipation an EURahmenprogrammen betrifft den überaus wichtigen Aspekt der Internationalisierung der Innovationsaktivitäten, und sie ist mit Kooperation und der Ausbildung internationaler Netzwerke verbunden, wobei es noch nicht genügend Erkenntnisse darüber gibt, in welchem Maße hier robuste, langfristig bestehende Beziehungen der Zusammenarbeit entstehen und welchen Impact das auf internationaler Ebene generierte Wissen auf das Vermögen und den Handlungsspielraum der nationalen Akteure hat. Tatsache ist, dass es eine Reihe von Institutionen gibt (z.B. im Bereich der außeruniversitären Sozialforschung), deren Erhalt fast ausschließlich von EU-Projekten abhängt.

\section{Abb. 13 - Anzahl der EU-Projekte mit Wiener Betelligung 2009 - 2013}

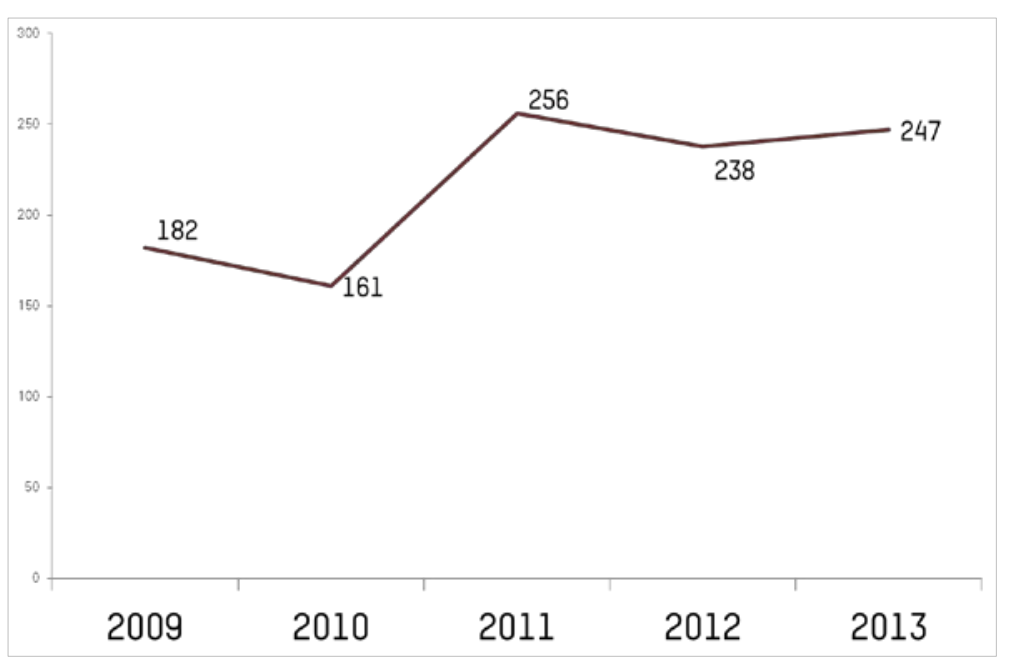

Datenquelle: CORDIS-Datenbank. N = 1.084 Projekte. Analyse: FASresearch

Die Entwicklung der EU-Projektzahlen mit Wiener Beteiligung hängt mit den Geltungszeiträumen der Rahmenprogramme sowie der Ausschreibungen zusammen; in den Jahren 2009 bis 2013 hat die Partizipation gemessen an den EU-Projekten, die sich in unseren 
CORDIS-Daten befinden, deutlich zugenommen. Auch die Forschungs- und Technologiefelder, in denen gearbeitet wird, haben mit den Schwerpunktsetzungen der Rahmenprogramme zu tun. Die folgende Tabelle listet die häufigsten Subjects der EU-Projekte auf, an denen Wiener Akteure (nach EU-Klassifikation Universitäten, außeruniversitäre Forschung, Unternehmen, Institutionen aus Politik und Verwaltung sowie sonstige Institutionen) in den Jahren 2009 bis 2013 teilnahmen: 
Tabelle 10 - Subjects der EU-Projekte mlt Wlener Betelligung 2009 - 2013 sowle der durchschnlttllchen Jährllchen Zunahme an Projekten („Momentum“)

\begin{tabular}{lccc} 
Subjects & Projekte & Prozent & Momentum \\
\hline Scientific Research & 340 & 21,0 & 9,9 \\
Life Sciences & 110 & 6,8 & 13,2 \\
Environmental Protection & 91 & 5,6 & $-0,3$ \\
Information and communication technology applications & 89 & 5,5 & 1,6 \\
Information, Media & 83 & 5,1 & 6,1 \\
Social Aspects & 75 & 4,6 & $-3,7$ \\
Education, Training & 70 & 4,3 & $-4,5$ \\
Medicine, Health & 60 & 3,7 & $-4,8$ \\
Coordination, Cooperation & 55 & 3,4 & $-6,1$ \\
Innovation, Technology Transfer & 54 & 3,3 & 0,6 \\
Transport & 50 & 3,1 & $-2,0$ \\
Biotechnology & 44 & 2,7 & 1,8 \\
Industrial Manufacture & 36 & 2,2 & 0,1 \\
Security & 34 & 2,1 & $-0,5$ \\
Network technologies & 33 & 2,0 & $-1,8$ \\
Medical biotechnology & 30 & 1,9 & $-2,2$ \\
Agriculture & 27 & 1,7 & 1,2 \\
Economic Aspects & 25 & 1,5 & $-2,1$ \\
Regional Development & 23 & 1,4 & 1,4 \\
Information Processing, Information Systems & 23 & 1,4 & $-3,4$
\end{tabular}

Datenquelle: CORDIS-Datenbank. $N=2.654$ Projekte. Analyse: FASresearch

An erster Stelle findet sich die (reichlich unklare) Kategorie "Scientific Research", mit der laut dem CORDIS Subject Index "General scientific and industrial research, or research not included elsewhere" bezeichnet ist. ${ }^{22}$ Es folgen die Life Sciences und die IKT innerhalb der vorderen Plätze, darüber hinaus fanden eine Vielzahl an Projekten in den Bereichen Umweltschutz, Information/Media sowie Social Aspects statt. Eine besonders starke Zunahme an Projekten im Zeitraum 2009 bis 2013 verzeichnen:

- Life Sciences $(+13,2)$

- Scientific Research $(+9,9)$

- Information, Media $(+6,1)$

- Biotechnologie $(+1,8)$

22 Vgl. http://cordis.europa.eu/guidance_old/sic-codes_en.html, abgerufen am 14.07.2015 
- Information and communication technology applications $(+1,6)$

- Regionalentwicklung $(+1,4)$

Die Verteilung der Arten von Institutionen, die an EU-Projekten beteiligt sind, spiegelt die währungsübergreifende Position wider, die die Rahmenprogramme in unserem Innovationsmodell einnehmen. Universitäten sind an 32,1\% der Projekte beteiligt, die außeruniversitäre Forschung an $27,8 \%$, Unternehmen an $21,9 \%$, Politik und Verwaltung an $12,5 \%$, die Zivilgesellschaft an 3,9\% sowie Kunst- und Kultureinrichtungen an 1,4\% der Projekte.

- Universitäten sind vor allem in den Bereichen Life Sciences bzw. Humanmedizin, Scientific Research, Food, Industrial Manufacture sowie Education und Training überproportional häufig vorhanden.

- Die außeruniversitäre Forschung finden wir bei Social Aspects, Policies, Biotechnologie, Energy Saving sowie Humanmedizin.

- Einen überdurchschnittlichen Unternehmensanteil stellen wir in den Bereichen Transport, Information Processing/Information Systems, Industrial Manufacture, Telekommunikation, Information and communication technology applications sowie Energietechnologien fest.

- Und die Zivilgesellschaft schließlich ist bei Social Aspects, Safety, Security, Innovation/Technologietransfer sowie Coordination/Cooperation überdurchschnittlich aktiv.

Sehen wir uns als Nächstes an, um welche Institutionen es sich dabei konkret handelt:

Tabelle 11 - Die Schlüsselspleler der Tellnahme an EU-Projekten 2009-2013

\begin{tabular}{lccc} 
Universität & EU-Projekte & Prozent & Momentum \\
\hline UNIVERSITÄT WIEN & 142 & 10,3 & 1,7 \\
TECHNISCHE UNIVERSITÄT WIEN & 136 & 9,9 & 4,2 \\
UNIVERSITÄT FÜR BODENKULTUR WIEN & 70 & 5,1 & 1,0 \\
MEDIZINISCHE UNIVERSITÄT WIEN & 47 & 3,4 & 0,6 \\
WIRTSCHAFTSUNIVERSITÄT WIEN & 25 & 1,8 & 0,8 \\
VETERINÄRMEDIZINISCHE UNIVERSITÄT WIEN & 11 & 0,8 & 0,6 \\
MODUL UNIVERSITY VIENNA GMBH & 4 & 0,3 & 0,2 \\
SIGMUND FREUD PRIVATUNIVERSITÄT WIEN & 4 & 0,3 & 0,0 \\
UNIVERSITÄT FÜR ANGEWANDTE KUNST WIEN & 1 & 0,1 & $-0,2$
\end{tabular}


Außeruniversitäre Forschung EU-Projekte Prozent Momentum

AIT AUSTRIAN INSTITUTE OF TECHNOLOGY GMBH

$\begin{array}{lll}88 & 6,4 & 2,7 \\ 51 & 3,7 & 0,8 \\ 32 & 2,3 & 1,1 \\ 15 & 1,1 & 0,0 \\ 13 & 0,9 & -0,6 \\ 12 & 0,9 & 0,7 \\ 11 & 0,8 & -0,6 \\ 9 & 0,7 & -0,3 \\ 9 & 0,7 & -0,6 \\ 9 & 0,7 & 0,7\end{array}$

ÖSTERREICHISCHE AKADEMIE DER WISSENSCHAFTEN

ZENTRUM FÜR SOZIALE INNOVATION

0,

0,7

\begin{tabular}{lccc} 
Unternehmen & EU-Proj ekte & Prozent & Momentum \\
\hline TTTECH COMPUTERTECHNIK AG & 11 & 0,8 & 0,8 \\
SIEMENS AG ÖSTERREICH & 9 & 0,7 & 0,0 \\
BOC ASSET MANAGEMENT GMBH & 7 & 0,5 & $-0,3$ \\
SERI - NACHHALTIGKEITSFORSCHUNGS UND -KOMMUNIKATIONS GMBH & 7 & 0,5 & $-0,2$ \\
BIOFACTION KG & 5 & 0,4 & 0,6 \\
EUTEMA TECHNOLOGY MANAGEMENT GMBH & 5 & 0,4 & $-0,1$ \\
FLUIDTIME DATA SERVICES GMBH & 5 & 0,4 & $-0,1$ \\
AEROSPACE \& ADVANCED COMPOSITES GMBH & 4 & 0,3 & 0,2 \\
BIOLUTION GMBH & 4 & 0,3 \\
BRIMATECH SERVICES GMBH & 4 & 0,3
\end{tabular}

Datenquelle: CORDIS-Datenbank. N = 2.654 Projekte. Analyse: FASresearch

Gemessen an der Zahl der Projekte in den Jahren 2009 bis 2013 weisen die TU Wien $(+4,2)$, das AIT $(+2,7)$, die Uni Wien $(+1,7)$, das ZSI $(+1,1)$, die BOKU $(+1)$ sowie die Firma TTTECH +0,8) das stärkste positive Momentum auf.

Aus der Zusammenarbeit in einer Vielzahl an EU-Projekten ergibt sich ein Kooperationsnetzwerk, das auf der folgenden Seite abgebildet ist. Die Netzwerkknoten stellen die Akteure dar, die Stärke einer Verbindung zwischen zwei Akteuren bezieht sich auf die Anzahl der gemeinsamen Partner, die Größe der Knoten verweist auf die Gesamtanzahl der Partner, mit denen ein Akteur jeweils verbunden ist, und die Farben schließlich repräsentieren Arten von Akteuren (Forschung, Wirtschaft, Sonstige). 
Abb. 14 - Netzwerk der Kooperation In EU-Projekten 2002 - 2014

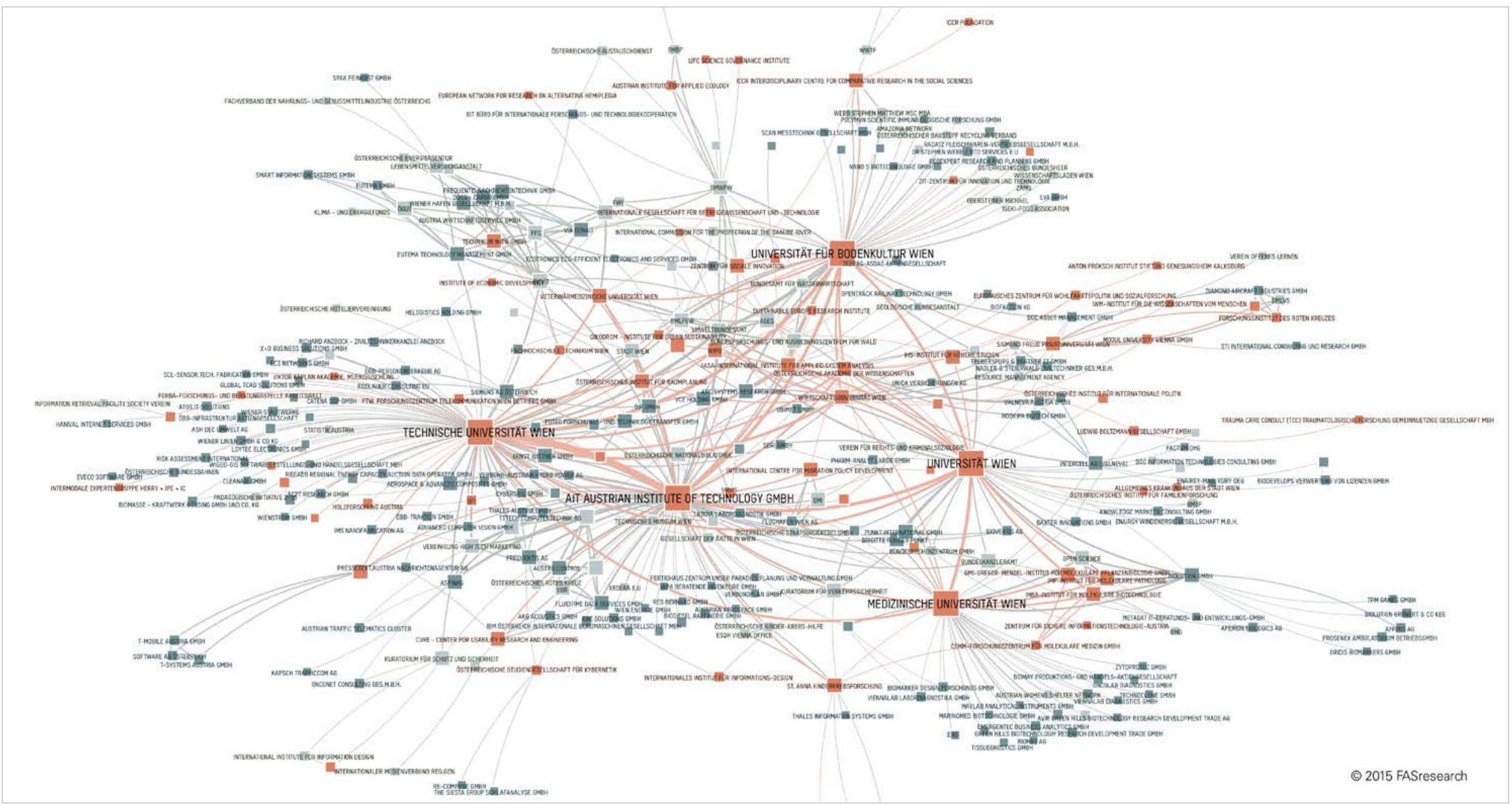

Datenquelle: CORDIS-Datenbank. N = 2.654 Projekte. Analyse und Grafik: FASresearch. Die Verbindungen zwischen den Institutionen zeigen die Kooperationen in EUProjekten an. Je stärker eine Linie, desto größer die Anzahl der gemeinsamen Projekte. Je größer eine Institution, desto größer die Anzahl der Kooperationspartner. 
Die Verbindung zwischen der TU Wien und dem AIT bildet die Hauptachse, zusammen mit der Universität Wien und der Universität für Bodenkultur ergibt sich eine Art Trapez, das das Rückgrat des Netzwerks bildet. Das AIT erscheint als der Hub, als DER NetzwerkKoordinator der gesamten Struktur, innerhalb derer sich auf der linken Seite die industrienahe Forschung (z.B. Transport, Energie, Nachrichtentechnik, Telekommunikation), oben die Regionalentwicklung, Umwelt- und Lebensmittelforschung sowie rechts unten die Life Sciences (Medizin und Biotechnologie) identifizieren lassen. Es sind die großen Universitäten (allerdings ohne die WU Wien, deren Anteil an den Wiener EU-Projekten 2009 - 2013 1,8\% beträgt; siehe Tabelle 11) und das AIT, die gemeinsam das Netzwerk aufspannen. Die Unternehmen, mit denen sie hauptsächlich kooperieren, siedeln sich rund um die Forschung an.

Aus den uns vorliegenden Daten lässt sich auch ein Netzwerk generieren, in dem die Institutionen mit den Forschungs- und Technologiefeldern verknüpft sind, in denen sie hauptsächlich EU-Projekte durchführen. Innerhalb dieser Verbindungsstruktur werden auf Basis der Vernetzungsdichte Cluster identifiziert, in denen sich Akteure mit ähnlichen Forschungsfeldern befinden. Auf diese Weise wird sichtbar, wer in welchen Forschungsfeldern EU-Projekte durchgeführt hat. Insgesamt ergeben sich durch diese Analyse sechs verschiedene Cluster mit folgenden Hauptakteuren:

1) Humanmedizin und Life Sciences - Meduni Wien, Vetmed, IMP, IMBA, Ludwig Boltzmann Gesellschaft, CEMM und Gregor Mendel Institut

2) Informations- und Kommunikationstechnologien - TU Wien, Siemens, FTW Forschungszentrum Telekommunikation Wien, Studiengesellschaft für Kybernetik, Telekom Austria, TTTECH

3) Energie und Infrastruktur - AIT, VIA DONAU, Kuratorium für Verkehrssicherheit, Atominstitut der österreichischen Universitäten, Andritz Hydro sowie Kapsch, Frequentis und ÖBB

4) Regionalentwicklung, Technologie- und Innovationstransfer - Zentrum für Soziale Innovation 
5) Umweltforschung - Universität für Bodenkultur, Umweltbundesamt, BMLFUW, IIASA, Bundesforschungszentrum für Wald, SERI

6) Scientific Research und Social Aspects - Universität Wien, Akademie der Wissenschaften, WU Wien, ICCR, IHS, WIIW, FORBA 
Zusammenfassung EU-Rahmenprogramme:

- Es besteht ein hohes sowie zunehmendes Maß an Partizipation Wiens an den EURahmenprogrammen - gute Voraussetzungen für die weitere Teilnahme sind gegeben (Horizon2020).

- Die Internationalisierung der Forschung ist (abgesehen von der finanziellen Förderung) als wesentliches „Asset” der Teilnahme an EU-Projekten zu betrachten.

- Die EU-Projekte fördern nicht nur den Aufbau eines internationalen Kooperationsnetzwerks, sondern stärken auch die Forschungskooperation innerhalb Wiens (siehe Abb. 14).

- Wirtschaft und Forschung erscheinen als gut eingebunden, Partizipation der Zivilgesellschaft (geringer Anteil an NGOs, Beschränkung auf bestimmte Bereiche) könnte gerade vor dem Hintergrund der Programmatik von Horizon2020 verstärkt werden.

- Life Sciences/Biotechnologie, IKT, Medien, Umweltforschung und Regionalentwicklung sind die Stärkefelder der Wiener Aktivitäten in EU-Rahmenprogrammen (Tab. 11).

- Die Zusammenarbeit zwischen der WU und der TU Wien liegt auch was die Rahmenprogramme anbelangt unter dem Durchschnitt. Auffällig ist auch die de facto Nicht-Beziehung zwischen der TU und der Uni Wien.

\subsection{Wiener Unternehmen in den Förderprogrammen der FFG}

Die Wirtschafts- und Forschungsfelder, in denen Wiener Unternehmen in den Jahren 2012, 2013 und 2014 Förderungen der FFG erhalten haben, liegen in Form von Subject Index Codes mit 45 Kategorien vor. Es handelt sich laut FFG ${ }^{23}$ um eine angepasste Versi-

23

https://www.ffg.at/sites/default/files/allgemeine_downloads/ffg\%20allgemein/publikationen/ffg_arb eitsprogramm_2013.pdf, Seite 6, abgerufen am 14.07.2015. 
on der EU-Cordis-Klassifikation (vgl. Fußnote 22). Lässt man die "kleinteiligen" Förderformate (z.B. Innovationsschecks) beiseite, kommt man auf insgesamt 1.227 Projekte. Aus den Daten lassen sich keine Kooperationsnetzwerke generieren, da uns keine projektbezogenen Daten vorliegen.

Die Förderungen werden in hohem Maße von kleinen und mittleren Unternehmen in Anspruch genommen; Kleinunternehmen waren an 64,9\%, mittlere Unternehmen an 6,8\% und große Unternehmen an 24,8\% der Projekte beteiligt (beim Rest auf 100\% liegen keine Angaben zur Unternehmensgröße vor).

Mehr als ein Fünftel der Projekte fallen in den Bereich „Oberflächenverkehr und technologien, fast 12\% auf IKT-Anwendungen, 9,8\% auf Informationsverarbeitung und systeme, 5,3\% auf Energietechnologien und 4,7\% auf Sicherheit. 
Tabelle 12 - SIC-Klassen der FFG nach der Anzahl der Projekte 2012 - 2014

\begin{tabular}{lccc} 
SIC & Gesamt & Prozent & Momentum \\
\hline OBERFL̈̈CHENVERKEHR UND -TECHNOLOGIEN & 254 & 20,7 & 3,00 \\
IKT-ANWENDUNGEN & 146 & 11,9 & $-2,50$ \\
INFORMATIONSVERARBEITUNG, INFORMATIONSSYSTEME & 120 & 9,8 & 0,00 \\
ENERGIESPEICHERUNG, -UMWANDLUNG UND TRANSPORT & 65 & 5,3 & $-0,50$ \\
SICHERHEIT & 58 & 4,7 & $-1,00$ \\
ENERGIEEINSPARUNG & 57 & 4,6 & $-9,50$ \\
BAUTECHNIK & 48 & 3,9 & 0,00 \\
MEDIZIN, GESUNDHEIT & 41 & 3,3 & $-5,00$ \\
ELEKTRONIK, MIKROELEKTRONIK & 37 & 3,0 & $-3,50$ \\
NACHHALTIGE ENTWICKLUNG & 32 & 2,6 & $-1,00$ \\
MEDIZINISCHE BIOTECHNOLOGIE & 30 & 2,4 & $-4,50$ \\
INDUSTRIELLE FERTIGUNG & 26 & 2,1 & $-0,50$ \\
BIOWISSENSCHAFTEN & 25 & 2,0 & $-1,00$ \\
WERKSTOFFTECHNIK & 25 & 2,0 & $-2,50$ \\
WELTRAUM & 19 & 1,5 & 2,50 \\
INFORMATION, MEDIEN & 12 & 1,0 & 0,00 \\
REGENERATIVE ENERGIETRÄGER & 12 & 1,0 & $-2,00$ \\
WIRTSCHAFTLICHE ASPEKTE & 12 & 1,0 & 1,50 \\
UMWELT & 11 & 0,9 & $-1,50$ \\
LUFTVERKEHR- UND TECHNOLOGIEN & 10 & 0,8 & $-1,00$
\end{tabular}

Datenquelle: Wirtschaftsagentur Wien. N = 1.227 FFG-Projekte. Analyse: FASresearch

21 der 45 Klassen (46,6\%) weisen in den Jahren 2012, 2013 und 2014 steigende Projektzahlen auf. Wenn wir nur die wichtigsten Klassen nehmen, z.B. die mit mindestens drei Projekten in den drei Jahren, finden wir ein besonders starkes Momentum bei:

- Oberflächenverkehr und -technologien (+3)

- Weltraum $(+2,5)$

- Wirtschaftliche Aspekte $(+1,5)$

- Unternehmensaspekte ${ }^{24}(+1)$

- Abfallwirtschaft $(+1)$

- Telekommunikation (+1)

\footnotetext{
24 "Research-oriented SMEs; High-tech SMEs; Privately-funded research organisations; R\&D competitiveness"; vgl. oben, Fußnote 22.
} 
Fallende Projektzahlen verzeichnen hingegen Energieeinsparung (-9,5), Medizin und Gesundheit (-5), Biotechnologie $(-4,5)$, „sonstige Energiethemen” (-4) und Elektronik, Mikroelektronik $(-3,5)$. 


\section{Die Wiener Patentlandschaft}

\subsection{Die Entwicklung der Patentzahlen Wiens}

Wenn wir uns nun den Patentaktivitäten Wiens zuwenden, gehen wir von der Sphäre der Exzellenz zu jener der ökonomischen Verwertung und des wirtschaftlichen Nutzens über. Es muss bedacht werden, dass die Patente nicht den gesamten Bereich der Verwertung von Innovationen abdecken; für viele Technologiefelder liefern sie jedoch umfassende Informationen über die Innovationsaktivitäten von Firmen, Forschungsinstitutionen oder Einzelpersonen. Sehen wir uns zunächst an, wie sich die Entwicklung der Patentzahlen von Wiener Anmeldern laut der uns vorliegenden Daten in den letzten Jahren entwickelt hat.

\section{Abb. 15 - Anzahl der Patente 1990 - 2013, Wien und übrige Bundesländer}

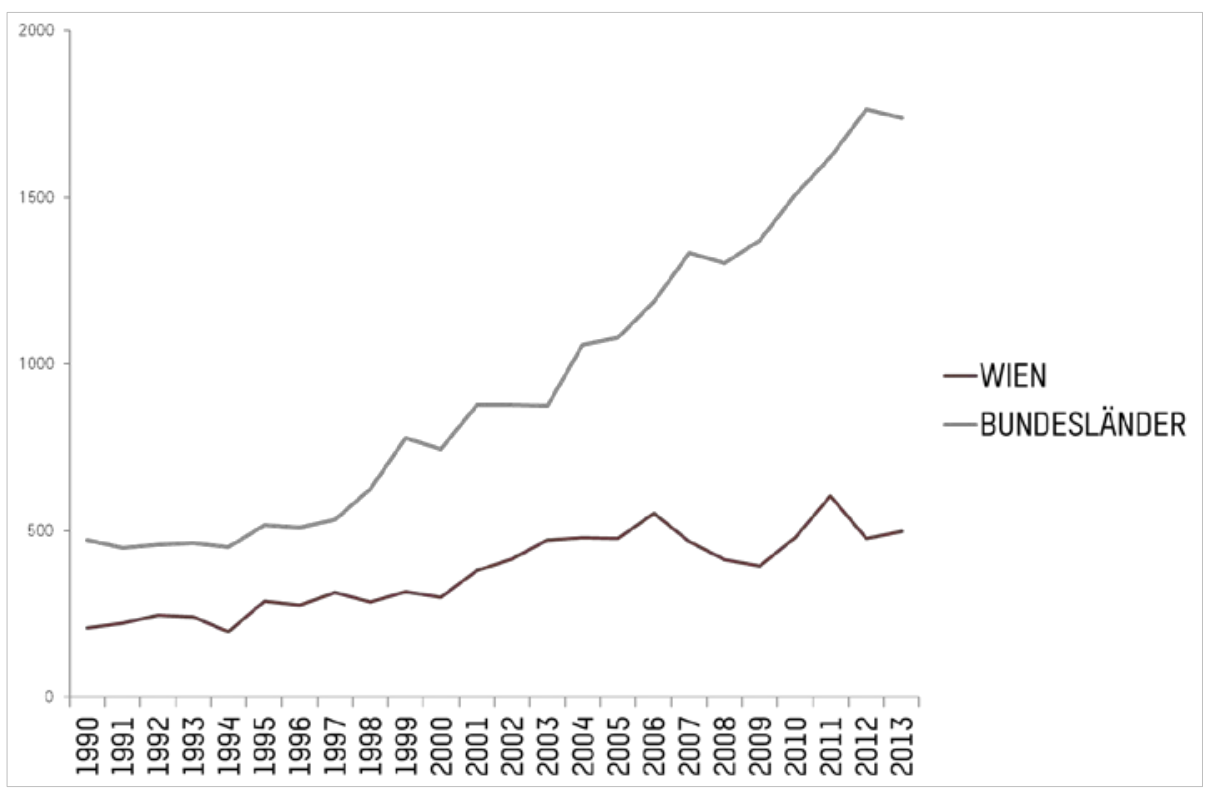

Datenquelle: Depatisnet, Espacenet. N = 31.618 Patente. Analyse: FASresearch

Das Diagramm beinhaltet die Anzahl der Patente, die von Wiener Akteuren in den Jahren 1990 bis 2013 angemeldet wurden, sowie jene von Anmeldern aus den anderen Bundesländern, und bezieht sich auf die Daten aus Depatisnet (Patentdatenbank des Deutschen Patent- und Markenamtes) und Espacenet (Patentdatenbank des Europäischen Patentamtes), d.h. auf die Patente mit österreichischer (Prefix "AT") und internationaler Gültigkeit (Prefix "EP" bzw. „WO”). In Summe geht es um 9.018 (Wien) und 22.600 Patente (übrige Bundesländer). 
Wien verzeichnet bis etwa zum Jahr 2006 steigende Patentzahlen, in der Folge gibt es einen Einbruch, der bis 2009 anhält, und ab dem Jahr 2010 zeichnet sich eine Erholung ab. Bei den Patentzahlen der anderen Bundesländer ist kein vergleichbarer Einbruch erkennbar. 1997 machten die Wiener Patente noch 37\% aller österreichischen Patente aus, 2007 sind es nur mehr 26\%, und 2013 waren es 22,3\%. Die Gründe haben wohl in erster Linie mit den Umstrukturierungen im Pharmabereich zu tun, wie etwa dem schrittweisen Rückzug der Firma Novartis, deren Österreich-Tochter früher besonders viele Patente angemeldet hat (im Jahr 2005 waren es 220), bis die Forschungsaktivitäten zurückgenommen wurden (2008 waren es nur mehr 41 Patente, 2014 gab es keine Anmeldungen mehr). Diese Annahme wird auch vom nächsten Diagramm untermauert, das zeigt, dass der Rückgang der Patentzahlen vor allem bei den Unternehmen stattfindet, während Universitäten und außeruniversitäre Forschung in dieser Hinsicht annähernd stabil blieben oder sogar wachsen:

\section{Abb. 16 - Entwicklung der Wiener Patentzahlen nach Anmelderkategorien (ohne Einzelpersonen) 2002 - 2013}

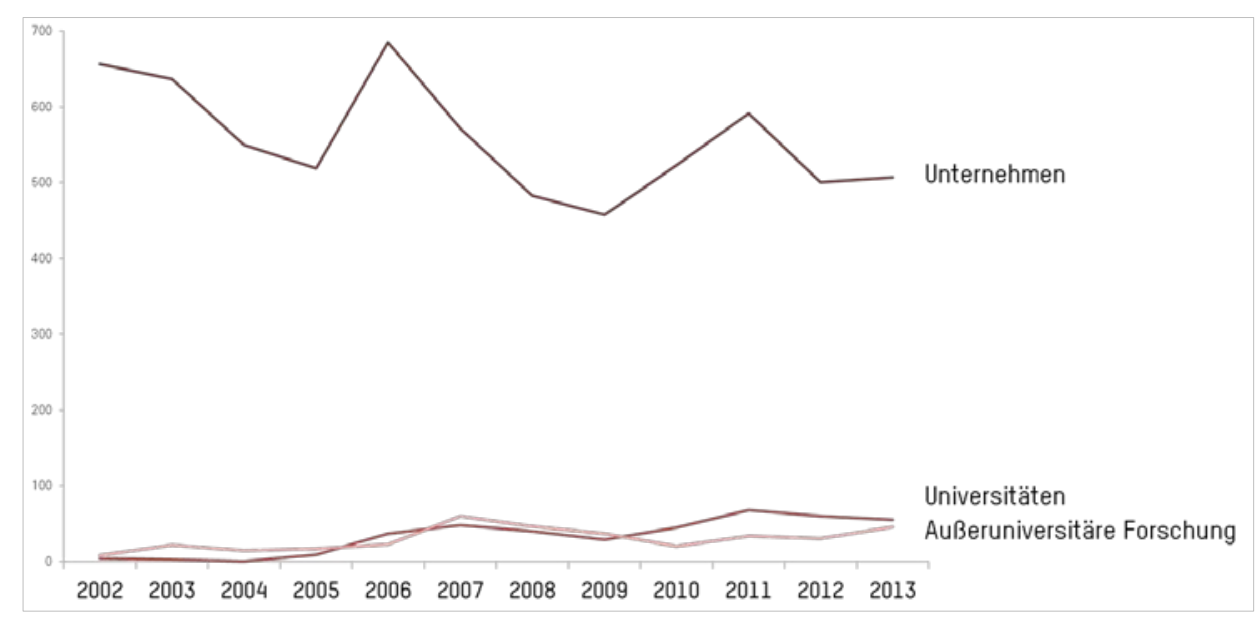

Datenquelle: Depatisnet, Espacenet. N = 7.443 Patente. Analyse: FASresearch

Wenn wir in einem nächsten Schritt zwischen Patenten mit österreichischer und Patenten mit internationaler Gültigkeit unterscheiden, kommen wir zu folgendem Bild:

Abb. 17 - Patente von Wlener Anmeldern mlt Internatlonaler und natlonaler Gültlgkelt 2002 - 2013 


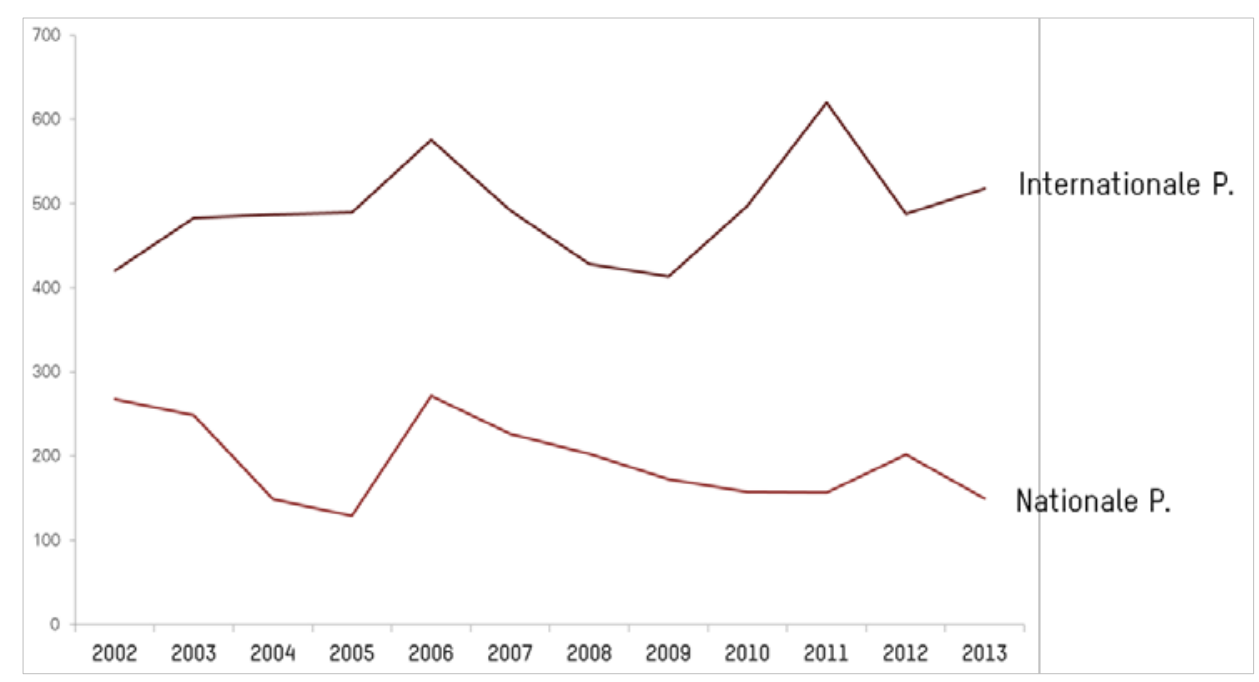

Datenquelle: Depatisnet, Espacenet. $N=8.681$ Patente. Analyse: FASresearch

Insgesamt ist es eine relativ geringe Zahl von großen Unternehmen mit vielen Patentanmeldungen, die die Gesamtentwicklung der Patentzahlen der Stadt Wien beeinflusst. Der Rückgang bei den nationalen Patenten ab etwa 2003 ist eng verbunden mit der Abnahme an Patenten bei der Firma Vaillant, der Einbruch ab etwa 2004 hat vor allem mit Novartis zu tun, und die neuerliche Steigerung ab 2012 ist in hohem Maße auf die Anmeldung internationaler Patente bei Borealis zurückzuführen.

Im Jahr 2013 wurden 22,9\% aller Patente in unserem Datensatz von Borealis angemeldet; die Top 5 Anmelder (Borealis, Siemens, TU Wien, AIT, Kapsch Trafficcom) vereinigen 39,7\% der Anmeldungen auf sich, und bei den Top 10 (neben den genannten sind es Affiris, EVVA, Refractory, MedUni Wien und Haas Food) sind es 48,4\% der Patente. Sehen wir uns noch an, wie sich die Konzentration auf wenige Anmelder in den letzten Jahren entwickelt hat: 


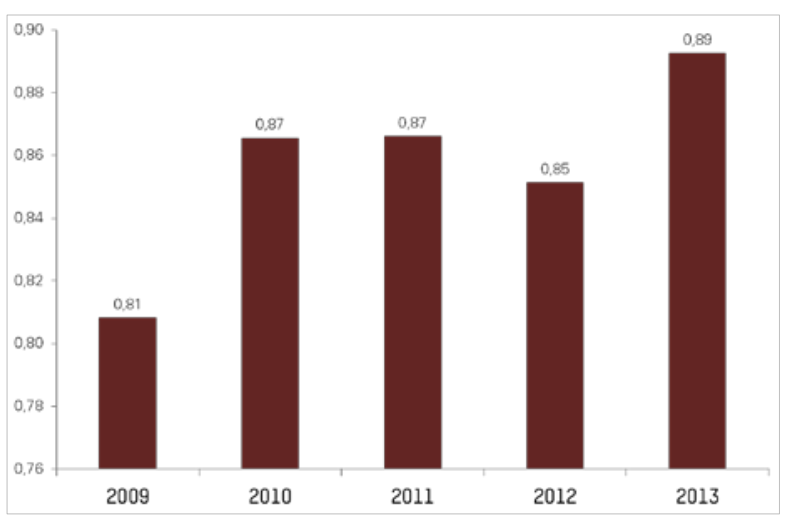

Datenquelle: Depatisnet, Espacenet. Analyse: FASresearch

Der Gini-Koeffizient gibt an, in welchem Maße sich die Patentzahlen auf einige wenige Anmelder konzentrieren; der Wert 1 würde bedeuten, dass alle Patente auf einen einzigen Anmelder fallen (maximale Konzentration). Wir sehen, dass die Konzentration in den letzten Jahren mit Ausnahme von 2012 kontinuierlich zugenommen hat.

Insgesamt stellen wir also fest,

- dass der Gesamttrend bei den Patenten in hohem Maße mit der Entwicklung bei einigen wenigen großen Anmeldern verbunden ist,

- dass Wien anteilsmäßig an den österreichweiten Patentzahlen verloren hat,

- dass dies insofern erstaunlich ist, als ja der Anteil Wiens in der Grundlagenforschung stabil geblieben ist, was auf ungenutzte Potentiale bei der Übersetzung von Grundlagenforschung in Anwendung bzw. Verwertung schließen lässt,

- dass sich die Entwicklung der Patentzahlen in den letzten fünf Jahren wieder stabilisiert hat, was vor allem auf die gesteigerte Anmeldung von internationalen $\mathrm{Pa}$ tenten zurückzuführen ist,

- und dass die Konzentration auf wenige Anmelder zugenommen hat.

\subsection{Die Technologiefelder der Patente}

Die Technologiebereiche, in denen patentiert wird, liegen in Form der Internationalen Patentklassifikation (IPC) vor, bei der es verschiedene Ebenen mit einer unterschiedlichen 
Anzahl von Kategorien gibt. Auf der Ebene der Klassen patentieren Wiener Anmelder in 106 verschiedenen Technologiefeldern, von denen die wichtigsten 20 im Folgenden aufgelistet sind:

Tabelle 13 - Technologlefelder nach der Zahl der Wiener Patente 2009 - 2013

\begin{tabular}{llcc} 
Klasse & Bezeichnung & Patente & Prozent \\
\hline A61 & Medizin oder Tiermedizin; Hygiene... & 580 & $13,0 \%$ \\
C08 & Organische makromolekulare Verbindungen; deren Her... & 541 & $12,1 \%$ \\
C07 & Organische Chemie... & 307 & $6,9 \%$ \\
G01 & Instrumente; Messen; Prüfen... & 259 & $5,8 \%$ \\
C12 & Biochemie; Bier; Spirituosen; Wein; Essig; Mikrobiologie... & 177 & $4,0 \%$ \\
H01 & Grundlegende elektrische Bauteile... & 172 & $3,8 \%$ \\
H04 & Elektrische Nachrichtentechnik... & 141 & $3,2 \%$ \\
B65 & Fördern; Packen; Lagern; Handhaben dünner oder fad... & 125 & $2,8 \%$ \\
G06 & Datenverarbeitung; Rechnen; Zählen... & 120 & $2,7 \%$ \\
F16 & Maschinenelemente oder -einheiten;... & 119 & $2,7 \%$ \\
E01 & Bauwesen; Straßen-, Eisenbahn-, Brückenbau... & 115 & $2,6 \%$ \\
B61 & Eisenbahnen... & 96 & $2,1 \%$ \\
H02 & Erzeugung etc. von elektrischem Strom & 92 & $2,1 \%$ \\
G07 & Kontrollvorrichtungen... & 86 & $1,9 \%$ \\
B01 & Physikalische oder chemische Verfahren ... & 80 & $1,8 \%$ \\
B29 & Verarbeiten von Kunststoffen... & 80 & $1,8 \%$ \\
G08 & Signalwesen... & 58 & $1,3 \%$ \\
E04 & Baukonstruktion... & 54 & $1,2 \%$ \\
A47 & Möbel; Haushaltsgegenstände oder -geräte; & 53 & $1,2 \%$ \\
B60 & Transportieren; Fahrzeuge allgemein... & 53 & $1,2 \%$
\end{tabular}

Datenquelle: Depatisnet, Espacenet. Analyse: FASresearch

Der wichtigste Bereich ist nach wie vor jener der Life Sciences; 13\% der Patente gab es in dieser Klasse. Es folgen die organischen makromolekularen Verbindungen (12,1\%), die organische Chemie (6,9\%), Instrumente, Messen, Prüfen (5,8\%) und Biochemie (4\%).

Wichtig ist hervorzuheben, dass die ersten beiden Bereiche völlig unterschiedlich strukturiert sind. Im Feld der Life Sciences (A61) gibt es insgesamt 151 Anmelder, wobei jener mit den meisten Patenten (Novartis Pharma) auf 18,8\% der Patente kommt. In der Klasse der organischen makromolekularen Verbindungen (C08) finden wir insgesamt 15 Anmelder, und der erste, nämlich Borealis, hält 94,2\% der Patentanmeldungen. Das heißt, die 
Life Sciences sind kleinteiliger strukturiert und versammeln eine Vielzahl von Anmeldern, während die Klasse C08 nur aufgrund eines einzelnen Anmelders so stark vertreten ist. Bei der drittgrößten Klasse, der organischen Chemie, gibt es wiederum 49 Anmelder, in der Klasse Instrumente, Messen, Prüfen sind es 88, und bei der Biochemie finden wir 51. Sehen wir uns für die zehn wichtigsten Klassen jeweils die Top-3 Anmelder an (es handelt sich insgesamt um 17 verschiedene Institutionen):

Tabelle 14 - Dle zehn häufigsten Patentklassen mlt den Jewells drel wlchtlgsten Wlener Anmeldern 2009 - 2013

\begin{tabular}{|c|c|c|}
\hline Klasse & Anmelder & Patente \\
\hline \multirow{3}{*}{ A61 } & NOVARTIS PHARMA GMBH & 164 \\
\hline & INTERCELL AG (VALNEVA) & 66 \\
\hline & MEDIZINISCHE UNIVERSITÄT WIEN & 49 \\
\hline \multirow{3}{*}{$\mathrm{C08}$} & BOREALIS AG & 730 \\
\hline & MONDI UNCOATED FINE \& KRAFT PAPER GMBH & 9 \\
\hline & TECHNISCHE UNIVERSITÄT WIEN & 8 \\
\hline \multirow{3}{*}{$\mathrm{CO7}$} & INTERCELL AG (VALNEVA) & 63 \\
\hline & NOVARTIS PHARMA GMBH & 60 \\
\hline & NABRIVA THERAPEUTICS AG & 26 \\
\hline \multirow{3}{*}{ G01 } & TECHNISCHE UNIVERSITÄT WIEN & 28 \\
\hline & SIEMENS AG ÖSTERREICH & 23 \\
\hline & AIT AUSTRIAN INSTITUTE OF TECHNOLOGY GMBH & 18 \\
\hline \multirow{3}{*}{ C12 } & INTERCELL AG (VALNEVA) & 40 \\
\hline & VALNEVA AUSTRIA GMBH & 19 \\
\hline & TECHNISCHE UNIVERSITÄT WIEN & 14 \\
\hline \multirow{3}{*}{$\mathrm{H} 01$} & BOREALIS AG & 85 \\
\hline & SIEMENS AG ÖSTERREICH & 10 \\
\hline & TECHNISCHE UNIVERSITÄT WIEN & 9 \\
\hline \multirow{3}{*}{$\mathrm{H} 04$} & KAPSCH TRAFFICCOM AG & 19 \\
\hline & AKG ACOUSTICS GMBH & 17 \\
\hline & T-MOBILE INTERNATIONAL AUSTRIA GMBH & 16 \\
\hline \multirow{3}{*}{ B65 } & MONDI AG & 29 \\
\hline & LUNATONE INDUSTRIELLE ELEKTRONIK GMBH & 12 \\
\hline & STARLINGER \& CO GMBH & 9 \\
\hline \multirow{3}{*}{ G06 } & TECHNISCHE UNIVERSITÄT WIEN & 28 \\
\hline & SIEMENS AG ÖSTERREICH & 23 \\
\hline & AIT AUSTRIAN INSTITUTE OF TECHNOLOGY GMBH & 18 \\
\hline F16 & BOREALIS AG & 41 \\
\hline
\end{tabular}




\begin{tabular}{|l|c|} 
HOERBIGER KOMPRESSORTECHNIK HOLDING GMBH & 27 \\
\cline { 2 - 3 } SIEMENS AG ÖSTERREICH & 7 \\
\hline
\end{tabular}

Datenquelle: Depatisnet, Espacenet. Analyse: FASresearch

Wie sieht es nun mit dem Momentum aus, d.h. mit der Entwicklung der Patentzahlen in den Klassen? Die folgenden Analysen beziehen sich wiederum auf die Jahre 2009 bis 2013, und wir beginnen mit der gröbsten Dimension der Internationalen Patentklassen, den Sektionen, bei denen es insgesamt acht Kategorien gibt:

- A - Täglicher Lebensbedarf

- B - Arbeitsverfahren; Transportieren

- C - Chemie; Hüttenwesen

- D - Textilien; Papier

- E - Bauwesen; Erdbohren; Bergbau

- F-Maschinenbau; Beleuchtung...

- G - Physik

- $\mathrm{H}$ - Elektrotechnik

Das folgende Diagramm bildet die Sektionen A bis $\mathrm{H}$ nach der Anzahl der Wiener Patente (y-Achse) sowie nach dem Momentum, d.h. der Entwicklung der Patentzahlen in den Jahren 2009 bis 2013 (x-Achse) ab.

Abb. 19 - Patent-Sektionen nach Größe und Momentum, Wiener Patente 2009 - 2013 


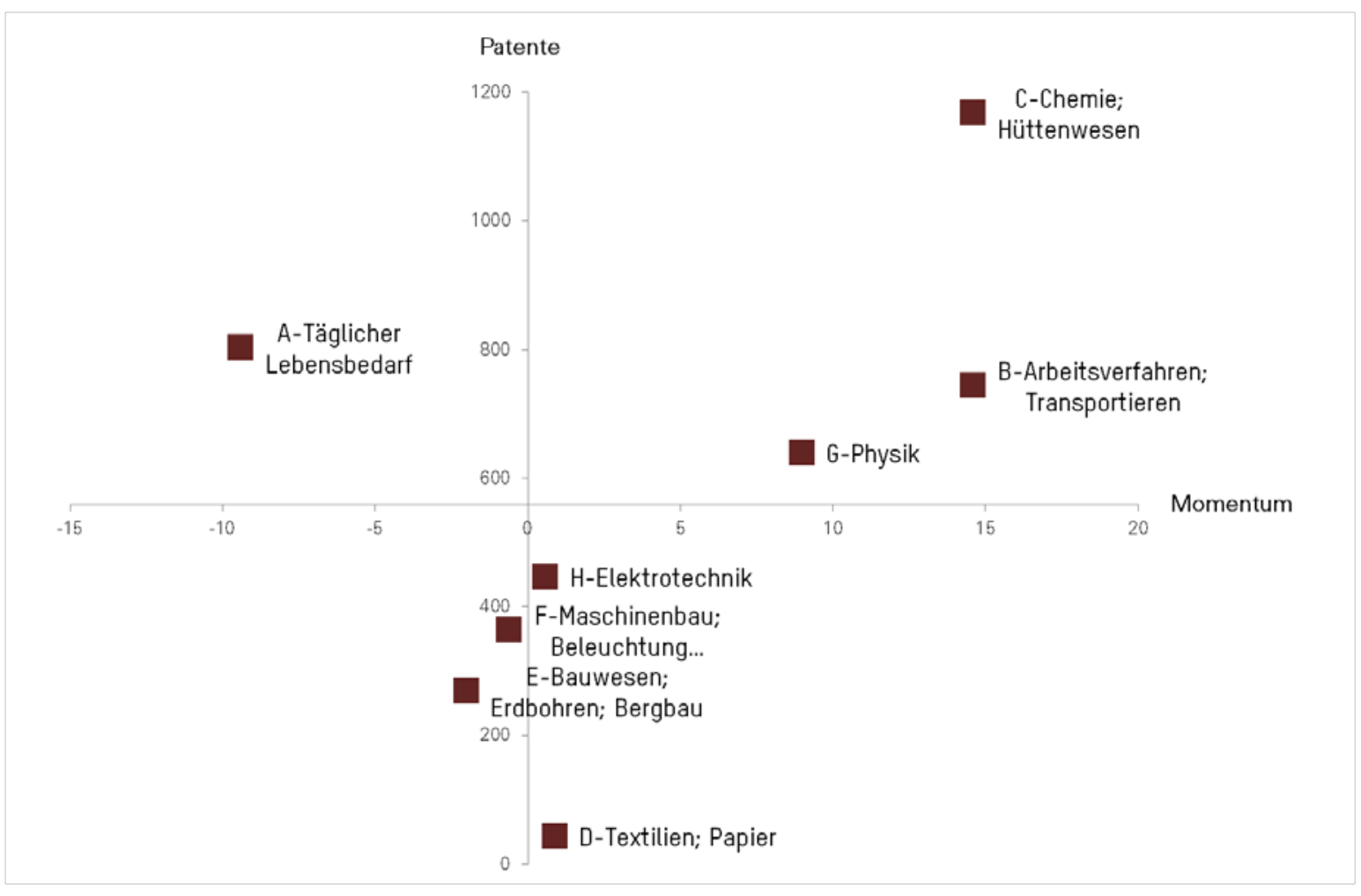

Datenquelle: Depatisnet, Espacenet. Analyse: FASresearch

Innerhalb der Sektionen gibt es deutliche Unterschiede zwischen den Klassen:

- Die Sektion A - Täglicher Lebensbedarf weist insgesamt ein negatives Momentum auf. Besonders betroffen davon sind die Klassen A61 Medizin sowie A01 Landwirtschaft. Zunehmende Patentzahlen gibt es hingegen bei A21 Backen, Herstellung von Teigen.

- Die Sektion B - Arbeitsverfahren, Transportieren zeigt ein positives Momentum. Besonders stark ist das Momentum bei B60 Transportieren, bei B32 Schichtkörper, bei B66 Heben, Schleppen sowie bei B61 Eisenbahnen.

- Die Sektion C - Chemie, Hüttenwesen weist ebenso ein positives Momentum auf, das auf die Klasse C08 zurückzuführen ist, in der Borealis so viele Patente angemeldet hat.

- Die Sektion D - Textilien, Papier beinhaltet nur eine geringe Zahl an Patenten, an der sich im Zeitverlauf wenig ändert. 
- Die Sektion E - Bauwesen ist stabil bis leicht rückläufig, wobei es zunehmende Patentzahlen bei Türen und Schlössern (E05, E06 Sicherheitstechnologien) und abnehmende bei E01 Bauwesen, Straßenbau, Brückenbau etc. gibt.

- Die Sektion F - Maschinenbau bleibt stabil. Insgesamt steigende Patentzahlen bei F03 Kraft- und Arbeitsmaschinen und bei F24 Heizung, Herde, Lüftung. Fallende Patentzahlen bei F23 Feuerungen, Verbrennungsverfahren und bei F16 Maschinenelemente.

- Die Sektion G - Physik besitzt ein positives Momentum, vor allem die Klassen G07 Kontrollvorrichtungen (und hier die Unterklasse G07B Fahrkartenausgeber; Fahrpreisanzeiger), G08 Signalwesen sowie G01 Instrumente; Messen; Prüfen... Rückläufig in dieser Sektion sind H02 Erzeugung, Umwandlung oder Verteilung von elektrischer Energie sowie H05 Elektrotechnik.

Welche sind nun die IPC-Klassen mit steigenden Patentzahlen in Wien, und wer sind die Anmelder?

Tabelle 15 - IPC-Klassen mlt dem stärksten Momentum, Wlener Patente 2009 bls 2013

\begin{tabular}{llcc} 
Klasse & Bezeichnung & Momentum & Patente \\
\hline C08 & Organische makromolekulare Verbindungen & 21,1 & 541 \\
G07 & Kontrollvorrichtungen... & 4,9 & 86 \\
B60 & Transportieren; Fahrzeuge allgemein... & 3,1 & 53 \\
G08 & Signalwesen... & 3,1 & 58 \\
G01 & Instrumente; Messen; Prüfen... & 2,5 & 259 \\
H04 & Elektrische Nachrichtentechnik... & 2,5 & 141 \\
A21 & Backen; Vorrichtungen zum Herstellen ... & 2,4 & 32 \\
B32 & Schichtkörper... & 2,2 & 51 \\
B66 & Heben; Anheben; Schleppen [Hebezeuge]... & 2,2 & 17 \\
B61 & Eisenbahnen... & 1,8 & 96
\end{tabular}

Datenquelle: Depatisnet, Espacenet. Analyse: FASresearch

Innerhalb dieser Klassen finden wir die folgenden Anmelder (jeweils die mit den meisten Patenten):

Tabelle 16 - Patentklassen mit dem größten Momentum 2009 - 2013 und den zugehörigen Wiener Anmeldern

\begin{tabular}{|l|l|c|}
\hline Klasse & Anmelder & Patente \\
\hline
\end{tabular}




\begin{tabular}{|c|c|c|}
\hline \multirow{3}{*}{$\mathrm{C08}$} & BOREALIS AG & 730 \\
\hline & MONDI UNCOATED FINE \& KRAFT PAPER GMBH & 9 \\
\hline & TECHNISCHE UNIVERSITÄT WIEN & 8 \\
\hline \multirow{3}{*}{ G07 } & KAPSCH TRAFFICCOM AG & 70 \\
\hline & EVVA SICHERHEITSTECHNOLOGIE GMBH & 9 \\
\hline & AIT AUSTRIAN INSTITUTE OF TECHNOLOGY GMBH & 3 \\
\hline \multirow{3}{*}{$\mathrm{B} 60$} & SIEMENS AG ÖSTERREICH & 13 \\
\hline & KAPSCH TRAFFICCOM AG & 7 \\
\hline & ÖBB-TECHNISCHE SERVICES GMBH & 4 \\
\hline \multirow{3}{*}{ G08 } & KAPSCH TRAFFICCOM AG & 36 \\
\hline & SIEMENS AG ÖSTERREICH & 3 \\
\hline & AIT AUSTRIAN INSTITUTE OF TECHNOLOGY GMBH & 1 \\
\hline \multirow{3}{*}{ G01 } & TECHNISCHE UNIVERSITÄT WIEN & 28 \\
\hline & SIEMENS AG ÖSTERREICH & 23 \\
\hline & AIT AUSTRIAN INSTITUTE OF TECHNOLOGY GMBH & 18 \\
\hline \multirow{4}{*}{$\mathrm{H} 04$} & KAPSCH TRAFFICCOM AG & 19 \\
\hline & AKG ACOUSTICS GMBH & 17 \\
\hline & T-MOBILE INTERNATIONAL AUSTRIA GMBH & 16 \\
\hline & TTTECH COMPUTERTECHNIK AG & 12 \\
\hline \multirow{2}{*}{ A21 } & HAAS FOOD EQUIPMENT GMBH & 22 \\
\hline & FRANZ HAAS WAFFEL-UND KEKSANLAGEN-INDUSTRIE GMBH & 12 \\
\hline \multirow{3}{*}{ B32 } & BOREALIS AG & 38 \\
\hline & MONDI AG & 4 \\
\hline & STARLINGER \& CO GMBH & 2 \\
\hline B66 & WAAGNER-BIRO AUSTRIA STAGE SYSTEMS AG & 4 \\
\hline \multirow{3}{*}{ B61 } & SIEMENS AG ÖSTERREICH & 72 \\
\hline & FRANZ PLASSER BAHNBAUMASCHINEN- INDUSTRIEGES. М.B.H. & 6 \\
\hline & PLASSER BAHNBAUMASCH FRANZ & 4 \\
\hline
\end{tabular}

Datenquelle: Depatisnet, Espacenet. Analyse: FASresearch

Die Klasse C08 (Organische makromolekulare Verbindungen) ist jene, in der Borealis hauptsächlich seine Patente anmeldet. Die Klasse G07 betrifft vor allem die Unterklasse Fahrkartenausgeber; Fahrpreisanzeiger; Frankiermaschinen, in der Kapsch Trafficcom aktiv ist. B60 betrifft Patente von Siemens, Kapsch und der ÖBB insbesondere in den Feldern Bremsen, Stromleitungen am Gleis sowie Signale. G08 (Kapsch, Siemens, AIT) beinhaltet vor allem Technologien im Bereich Steuerung und Überwachen des Verkehrs. Und in G01 (Instrumente, Messen, Prüfen) sind die TU Wien, Siemens und das AIT stark vertreten.

Die Patentklassen mit fallenden Patentzahlen sind hingegen die folgenden: 
Tabelle 17 - Patentklassen mit fallenden Patentzahlen, Wiener Patente 2009 - 2013

\begin{tabular}{llcc} 
Klasse & Bezeichnung & Momentum & Patente \\
\hline A61 & Medizin oder Tiermedizin; Hygiene... & $-6,9$ & 580 \\
C07 & Organische Chemie... & $-4,2$ & 307 \\
A01 & Landwirtschaft; Forstwirtschaft; Tierzucht; Jagen;... & $-2,8$ & 47 \\
& Erzeugung, Umwandlung oder Verteilung von & & \\
H02 & elektris... & $-2,1$ & 92 \\
E01 & Bauwesen; Straßen-, Eisenbahn-, Brückenbau... & $-1,9$ & 115 \\
F16 & Maschinenelemente oder -einheiten & $-1,6$ & 119 \\
G10 & Musikinstrumente; Akustik... & $-1,1$ & 30 \\
B22 & Gießerei; Pulvermetallurgie... & $-0,6$ & 36 \\
B23 & Werkzeugmaschinen; Metallbearbeitung, soweit nicht... & $-0,6$ & 31 \\
C09 & Farbstoffe; Anstrichstoffe; Polituren; Naturharze;... & $-0,6$ & 35
\end{tabular}

Datenquelle: Depatisnet, Espacenet. Analyse: FASresearch

Mit der Medizin (A61) weist ein Technologiefeld, in dem Wien, wie wir gesehen haben, bereichsübergreifend sehr stark ist (siehe Grundlagenforschung, Publikationen und Anwendung), stagnierende bis sinkende Patentzahlen auf, was auf technologiezyklusbedingte Sättigungseffekte hinweisen und, wie oben bemerkt, besonders mit dem Wegfall großer Unternehmen zu tun haben dürfte. Denn andererseits gibt es im Bereich der Life Sciences in Wien nach wie vor sehr viele, eher kleine bis mittelgroße Akteure, die weiterhin Patente hervorbringen (Valneva, Nabriva Therapeutics, Affiris, Biomay). Es scheint, was den Patentoutput angeht, ein gewisses Maß an Sättigung im Bereich der Life Sciences und der Biotechnologie zu geben, worauf auch die zweite Klasse in der Liste hinweist, die organische Chemie (CO7), in der ähnliche Unternehmen vertreten sind wie in der Klasse der Medizin (A61). Die Klasse A01 (Landwirtschaft) stellt insgesamt nur einen kleinen Bereich dar; Hauptanmelder hier sind das Gregor-Mendel-Institut, die TU Wien und das AIT. Der Rückgang der Patente in diesem Feld hat möglicherweise mit der Insolvenz eines einzelnen Unternehmens (Aka Technologies) im Jahre 2009 zu tun. H02 betrifft die Erzeugung oder Verteilung von elektrischer Energie, und die wichtigsten Player in diesem Technologiefeld sind Siemens, Lunatone Industrielle Elektronik, die TU Wien sowie die FH Technikum Wien. Im Bauwesen (E01) schließlich geht es um Patentanmelder wie Franz Plasser Bahnbaumaschinen (jetzt Plasser \& Theurer) und die Semperit AG Holding, 
die sich auf andere Technologiefelder spezialisiert haben (Gummi- und Kunststoffprodukte für die Medizin und die Industrie).

\subsection{Das Alter der Patente in den Technologiefeldern}

Anhand des Bewilligungsdatums der Patente lässt sich deren Durchschnittsalter berechnen. Dies kann dann auf die Technologiebereiche übertragen werden. Die folgende Tabelle zeigt die wichtigsten (mindestens fünf Patente 1979 - 2014) Technologiefelder in Wien gereiht nach dem Durchschnittsalter der Patente sowie die zugehörigen Patentanmelder:

Tabelle 18 - Technologlefelder mlt den Patenten Wlener Anmelder 1979 - 2014 mlt dem gerlngsten Durchschnlttsalter; nur Unterklassen mlt mIndestens fünf Patenten Im angegebenen Zeltraum

\begin{tabular}{|c|c|c|c|c|}
\hline Unterklasse & Bezeichnung & Anmelder & Patente & $\begin{array}{c}\text { Durchschnitts- } \\
\text { alter } \\
\end{array}$ \\
\hline H04W & Drahtlose Kommunikationsnetze & $\begin{array}{l}\text { T-Mobile Austria, Kapsch } \\
\text { Trafficcom, Siemens }\end{array}$ & 36 & 3,3 \\
\hline G06N & $\begin{array}{l}\text { Rechnersysteme, basierend auf } \\
\text { spezifischen Rechenmodellen }\end{array}$ & AIT, Cortical, Siemens & 5 & 4,4 \\
\hline G07B & $\begin{array}{l}\text { Fahrkartenausgeber; Fahrpreisan- } \\
\text { zeiger; Frankiermaschinen }\end{array}$ & Kapsch Trafficcom & 79 & 5,3 \\
\hline $\mathrm{B} 25 \mathrm{H}$ & $\begin{array}{l}\text { Werkstatteinrichtungen, z.B. zum } \\
\text { Anreißen von Werkstücken... }\end{array}$ & $\begin{array}{l}\text { WZV - Werkzeugvertriebs } \\
\text { GmbH }\end{array}$ & 13 & 5,3 \\
\hline CO8K & $\begin{array}{l}\text { Verwendung von anorganischen } \\
\text { oder nichtmakromolekularen orga- } \\
\text { nischen Stoffen... }\end{array}$ & Borealis AG & 169 & 5,3 \\
\hline H01B & Kabel; Leiter; Isolatoren & Borealis AG & 105 & 5,3 \\
\hline A01P & Biozide Wirkung & $\begin{array}{l}\text { Gregor Mendel-Institut, } \\
\text { TU Wien }\end{array}$ & 12 & 5,4 \\
\hline C09J & $\begin{array}{l}\text { Klebstoffe; Klebeverfahren allge- } \\
\text { mein }\end{array}$ & $\begin{array}{l}\text { Agrana Stärke GmbH, } \\
\text { Borealis AG }\end{array}$ & 9 & 5,4 \\
\hline B82Y & Nanotechnologie & IMS Nanofabrication AG & 6 & 5,5 \\
\hline G06Q & $\begin{array}{l}\text { Datenverarbeitungsvorrichtungen } \\
\text { oder -verfahren }\end{array}$ & $\begin{array}{l}\text { AIT, EVVA Sicherheits- } \\
\text { technologien, Siemens }\end{array}$ & 40 & 5,6 \\
\hline
\end{tabular}


Es ist deutlich erkennbar, dass sich die „jüngeren“ Patente (mit geringerem Durchschnittsalter) vor allem in den Bereichen IKT, Datenverarbeitung, Verkehrssysteme, aber auch in der Chemie und Kunststofftechnologie befinden. „Ältere” Patente finden sich hingegen in Patentklassen wie C13 (Zuckerindustrie), B28 (Zementverarbeitung), F42 (Munition, Sprengen), E21 (Bergbau), F22 (Dampferzeugung) oder A22 (Metzgerei).

\subsection{Die Schlüsselspieler bei den Patentanmeldungen}

71,9\% aller Wiener Patente im Zeitraum 2009 - 2013 wurden von Unternehmen angemeldet; 15,7\% von Einzelpersonen; 7,2\% von Universitäten; 4,7 \% von der außeruniversitären Forschung, der Rest fällt auf sonstige Institutionen. Die folgenden Tabellen zeigen die Unternehmen sowie die Forschung (Universitäten plus außeruniversitäre Forschung) mit den meisten Patenten in den Jahren 2009 - 2013 sowie der Patententwicklung (Momentum):

Tabelle 19 - Unternehmen mit Sitz in Wien nach der Zahl der Patente 2009 -2013

\begin{tabular}{lccc} 
Unternehmen & Patente & Prozent & Momentum \\
\hline BOREALIS AG & 573 & $16,0 \%$ & 24,7 \\
SIEMENS AG ÖSTERREICH & 166 & $4,6 \%$ & $-0,9$ \\
NOVARTIS PHARMA GMBH & 110 & $3,1 \%$ & $-5,4$ \\
KAPSCH TRAFFICCOM AG & 88 & $2,4 \%$ & 5,5 \\
INTERCELL AG (VALNEVA) & 71 & $2,0 \%$ & $-1,4$ \\
REFRACTORY INTELLECTUAL PROPERTY GMBH \& CO KG & 47 & $1,3 \%$ & $-0,4$ \\
HOERBIGER KOMPRESSORTECHNIK & 47 & $1,3 \%$ & $-0,7$ \\
FRANZ PLASSER BAHNBAUMASCHINEN & 45 & $1,3 \%$ & 0,3 \\
PLASSER BAHNBAUMASCH FRANZ & 37 & $1,0 \%$ & $-1,5$ \\
EVVA SICHERHEITSTECHNOLOGIE GMBH & 36 & $1,0 \%$ & 1,7 \\
VAILLANT GROUP AUSTRIA GMBH & 36 & $1,0 \%$ & 3,4 \\
MONDI AG & 34 & $0,9 \%$ & 0,9 \\
LUNATONE INDUSTRIELLE ELEKTRONIK GMBH & 34 & $0,9 \%$ & $-0,4$ \\
STARLINGER \& CO GMBH & 33 \\
AFFIRIS AG & 33 & $0,9 \%$ & $-0,3$ \\
SEMPERIT AG HOLDING & 31 & $0,9 \%$ & 2,6 \\
BIOMAY AG & 29 & $0,8 \%$ & $-1,5$ \\
VALNEVA AUSTRIA GMBH & 25 & $0,7 \%$ & 0,4 \\
VAILLANT AUSTRIA GMBH & 25 & $0,7 \%$ & 0,5
\end{tabular}


Datenquelle: Depatisnet, Espacenet. Analyse: FASresearch 
Tabelle 20 - Forschungsinstitutionen mit SItz in Wien nach der Zahl der Patente 2009 -2013 (Depatisnet, Espacenet)

\begin{tabular}{lccc} 
Anmelder & Patente & Prozent & Momentum \\
\hline TECHNISCHE UNIVERSITÄT WIEN & 154 & $4,3 \%$ & 5,5 \\
AIT AUSTRIAN INSTITUTE OF TECHNOLOGY GMBH & 67 & $1,9 \%$ & 3,8 \\
MEDIZINISCHE UNIVERSITÄT WIEN & 48 & $1,3 \%$ & 0,9 \\
UNIVERSITÄT FÜR BODENKULTUR WIEN & 20 & $0,6 \%$ & $-0,3$ \\
UNIVERSITÄT WIEN & 20 & $0,6 \%$ & 0,1 \\
VETERINÄRMEDIZINISCHE UNIVERSITÄT WIEN & 15 & $0,4 \%$ & 0,5 \\
IMBA-INSTITUT FÜR MOLEKULARE BIOTECHNOLOGIE & 13 & $0,4 \%$ & 1,0 \\
FACHHOCHSCHULE TECHNIKUM WIEN & 12 & $0,3 \%$ & 0,6 \\
IMG INSTITUT FÜR MEDIZINISCHE GENOMFORSCHUNG & 9 & $0,3 \%$ & 0,8 \\
ÖSTERREICHISCHE AKADEMIE DER WISSENSCHAFTEN & 9 & $0,3 \%$ & $-0,3$ \\
CEMM-FORSCHUNGSZENTRUM & 5 & $0,1 \%$ & 0,3 \\
LUDWIG BOLTZMANN GESELLSCHAFT GMBH & 4 & $0,1 \%$ & 0,0 \\
GMI-GREGOR-MENDEL-INSTITUT & 4 & $0,1 \%$ & $-0,6$ \\
VRVIS ZENTRUM FÜR VIRTUAL REALITY & 3 & $0,1 \%$ & 0,4 \\
IMP-INSTITUT FÜR MOLEKULARE PATHOLOGIE & 2 & $0,1 \%$ & 0,4 \\
LUDWIG BOLTZMANN CLUSTER FÜR KARD. FG. & 2 & $0,1 \%$ & 0,0 \\
TRAUMA CARE CONSULT (TCC) & 2 & $0,1 \%$ & $-0,2$ \\
FH CAMPUS WIEN & 2 & $0,1 \%$ & $-0,2$
\end{tabular}

Datenquelle: Depatisnet, Espacenet. Analyse: FASresearch

Die Wiener Anmelder mit dem stärksten positiven Momentum 2009 - 2013 sind:

- $\quad$ BOREALIS AG $(+24,7)$

- TECHNISCHE UNIVERSITÄT WIEN $(+5,5)$

- $\quad$ KAPSCH TRAFFICCOM AG $(+5,5)$

- $\quad$ Ait Austrian institute OF TECHNOLOGY GMBH $(+3,8)$

- VAillant GROUP AUSTRIA GMBH $(+3,4)$

- AFFIRIS AG $(+2,6)$

- HAAS FOOD EQUIPMENT GMBH $(+2,5)$

- EVVA SICHERHEITSTECHNOLOGIE GMBH $(+1,7)$

- SIEMENS CONVERGENCE CREATORS GMBH $(+1,6)$

- T-MOBILE INTERNATIONAL AUSTRIA GMBH $(+1,3)$ 


\section{5. Das Zitationsnetzwerk der Wiener Patentlandschaft}

Die Anzahl der Patente allein gibt noch nicht ausreichend Auskunft über die Innovationsaktivitäten einer Stadt im Bereich der wirtschaftlichen Verwertung. Worauf es zusätzlich ankommt, ist, in welchem Maße die Patente in Zitationsbeziehungen eingebettet sind. Anmelder verweisen auf den Stand der Technologie, die für ihr Patent relevant ist, indem sie Fachliteratur und andere Patente zitieren. Zitationen können demnach als Indikator für die Bedeutung von Patenten aufgefasst werden, und Zitate zeugen von Wissens- und Technologietransfers.

Patente, die miteinander durch Zitationsbeziehungen verbunden sind, ergeben Netzwerke, die mit den Instrumenten der sozialen Netzwerkanalyse untersucht werden können. Doch interessiert nicht eigentlich das Netzwerk der Patente. Diese sind erstens ja mit den IPC-Technologieklassen verbunden. Das heißt, aus den Daten mit Patenten und Zitaten können auch Netzwerke der Patentklassen, die einander zitieren, generiert werden. Es handelt sich dann um Technologiefelder, zwischen denen offensichtlich kraft der Zitationen ein Transfer von Wissen und Know-how stattfindet. Und zweitens kennen wir die Anmelder der Patente und können daher auch Netzwerke erzeugen, die aus Patentanmeldern bestehen, die miteinander dadurch verbunden sind, dass sie ihre Patente wechselseitig zitieren (Wissenstransfer zwischen Akteuren). Auf diese beiden Netzwerke (Technologieklassen und Anmelder) beziehen sich die folgenden Analysen.

\subsubsection{Das Zitationsnetzwerk der Patentklassen}


Für den gesamten Analysezeitraum 1979 bis 2014, für den uns Patentdaten in digitalisierter Form zur Verfügung stehen, finden wir 7.481 Wiener Patente ${ }^{25}$, die andere Patente zitieren bzw. zitiert werden - nämlich insgesamt 21.709 Patenten außerhalb Wiens.

Wenn wir nun diese Patente mit ihren Patentklassen verknüpfen (und die Patente selbst weglassen, vgl. Abb. 20), ergibt sich ein neues Netzwerk, das nunmehr aus den Patentklassen besteht, die miteinander durch Zitationsbeziehungen verbunden sind.

${ }^{25}$ Patente mit internationaler Gültigkeit aus Espacenet, der Patentdatenbank des Europäischen Patentamts. 


\section{Abb. 20 - Umwandlung des Patentnetzwerks In eln Patentklassennetzwerk}
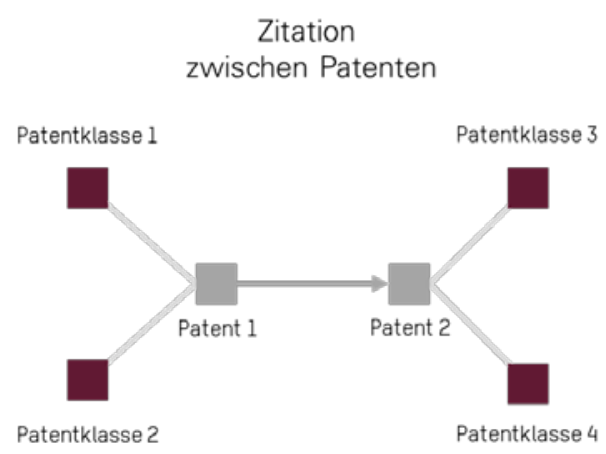

Zitation zwischen Patentklassen

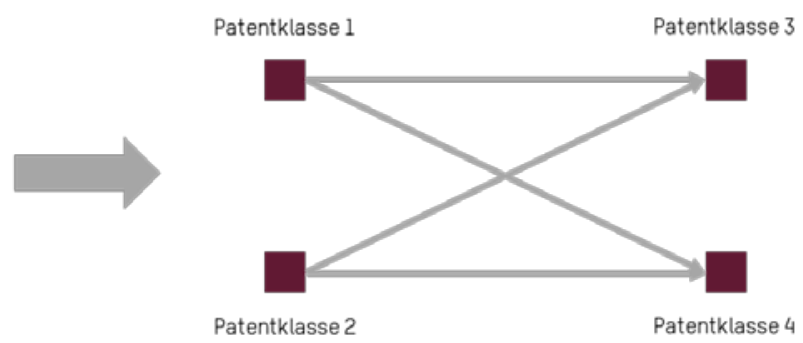

Grafik: FASresearch

Insgesamt sind es 567 IPC-Klassen (auf der Ebene der Unterklassen) und 8.196 Zitationsbeziehungen. Es handelt sich um ein sehr dichtes Netzwerk, d.h. es gibt sehr viele Beziehungen bei einer vergleichsweise geringen Zahl an Knoten. Um die darin bestehenden Strukturen besser erkennen zu können, muss man die Anzahl der Beziehungen reduzieren, sodass nur die wichtigsten im Netzwerk verbleiben. Die Zitationsbeziehungen haben nämlich einen Wert, der etwas über ihre Stärke aussagt - die Anzahl der Patente. Es gibt Patentklassen, die durch sehr viele Patente miteinander verbunden sind, und solche, bei denen es nur wenige Patente sind. Wir wählen nun für jede Patentklasse die drei wichtigsten Patentklassen aus, die sie zitiert, und zwar gemessen an der Anzahl der Patente, die die Verbindungen zwischen ihnen herstellen.

Die Patentklasse A61K (Präparate für medizinische [...] Zwecke) zum Beispiel zitiert insgesamt 136 andere Patentklassen, die drei wichtigsten nach der Zahl der Patente sind C07D (Heterozyklische Verbindungen), A61K (Therapeutische Aktivität von chemischen Verbindungen oder medizinischen Zubereitungen) und C07C (Acyclische oder carbocyclische Verbindungen). Diese drei von A61K ausgehenden Beziehungen belassen wir, alle anderen Zitationen fallen weg. Auf diese Weise wird gewissermaßen das Rückgrat des Zitationsnetzwerks sichtbar.

Das Resultat dieser Reduktion ist ein Netzwerk mit 331 Patent-Unterklassen und 780 Zitationsbeziehungen, in dem Cluster von Technologiefeldern deutlich erkennbar sind. Die Abb. 22 zeigt die Visualisierung dieses Netzwerks. Da es auf allen Zitationen im Zeitraum 
1979 bis 2014 basiert, enthält es gewissermaßen auch die Vergangenheit der Patentaktivitäten Wiens. Die Größe der Knoten in der Darstellung aber bezieht sich allein auf die Anzahl der Wiener Patente von 2009 bis 2013, und die Farben auf das Momentum, d.h. auf die Entwicklung der Patentzahlen in eben diesem Zeitraum (Orange bedeutet positives Momentum, Grün negatives, Grau stabile Patentzahlen).

Im Zentrum des Netzwerks befindet sich deutlich erkennbar ein in sich dicht vernetzter Cluster mit Patentklassen der Humanmedizin (A61K, A61P), der Organischen Chemie (C07D, C07K) sowie der Biochemie (C12N). Die Position im Netzwerk zeigt, dass es sich um die zentralen Forschungsfelder handelt, in denen in Wien patentiert wird. Gleichzeitig weisen genau diese Patentklassen eher rückgängige Patentzahlen auf; das heißt, das Zentrum wächst nicht mehr. Oberhalb des Zentrums befindet sich hingegen ein Cluster mit wachsenden Patentfeldern aus dem Bereich der Chemie (C08 Organische makromolekulare Verbindungen). Rund um das Zentrum herum, also an der Semiperipherie und Peripherie des Netzwerks, sind weitere Cluster von für Wien wichtige Patentklassen angesiedelt, zum einen solche, in denen die Patentzahlen eher abnehmen (Elektrotechnik, Verbrennung und Dampferzeugung, Heizung/Kühlung/Lüftung), und andere mit steigenden Patentzahlen (Nachrichtentechnik, Eisenbahnen). 


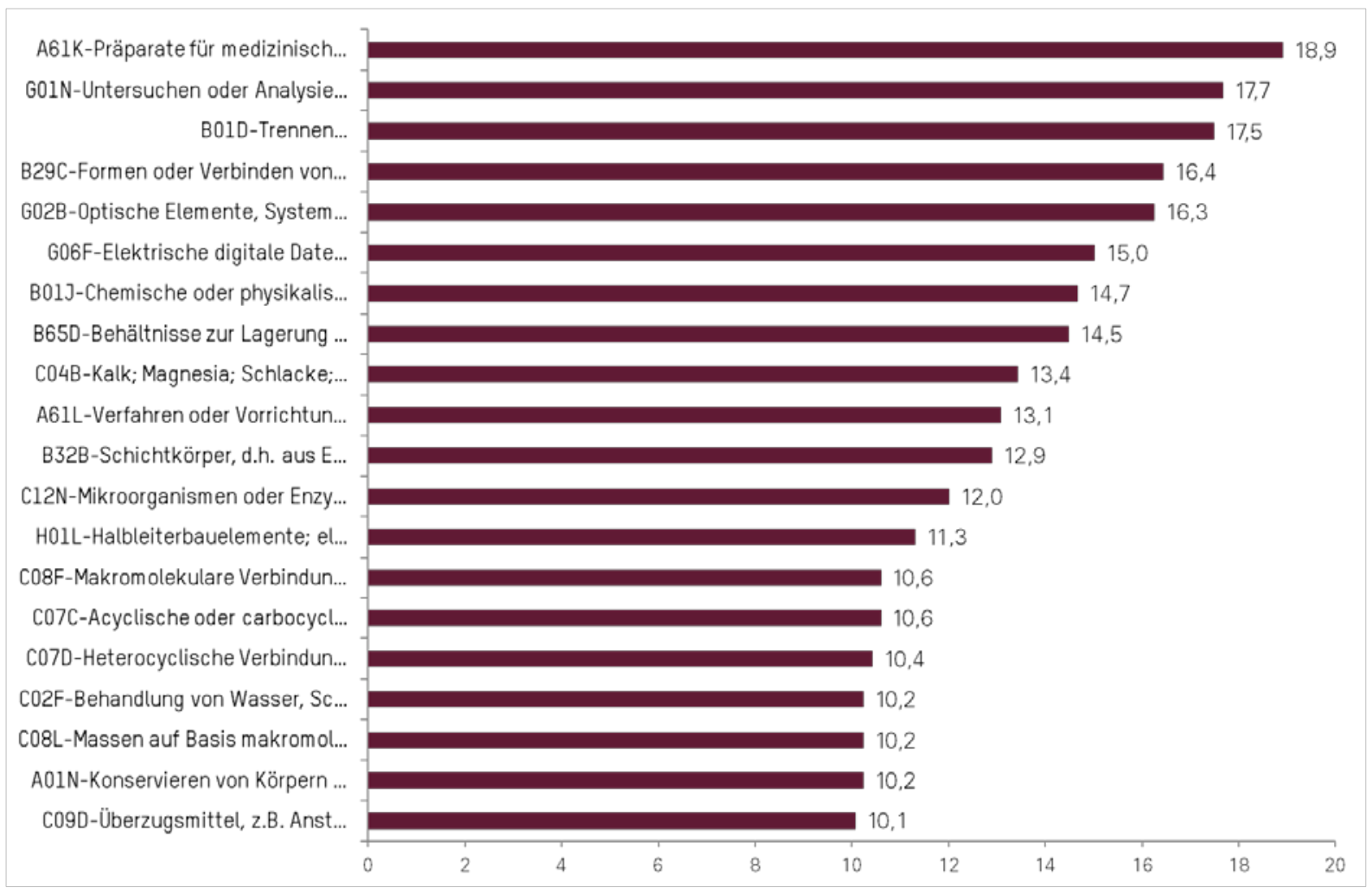

Datenquelle: Espacenet. Analyse: FASresearch

Die Liste der Patentklassen, die von den meisten anderen Klassen zitiert werden (unabhängig davon, durch wie viele Patente), spiegelt die Schwerpunkte der Wiener Patentlandschaft wider: Humanmedizin, Chemie sowie Informations- und Kommunikationstechnologien. Die Werte im folgenden Diagramm (Abb. 21) sind Prozente und beziehen sich darauf, von wieviel Prozent aller anderen Klassen eine Patentklasse zitiert wird (z.B. A61K wird von 107 der 567 Patent-Unterklassen zitiert, das sind 18,9\%). 
Abb. 22 - Zltatlonsnetzwerk der Patentklassen, Wlener Patente 1979 - 2014

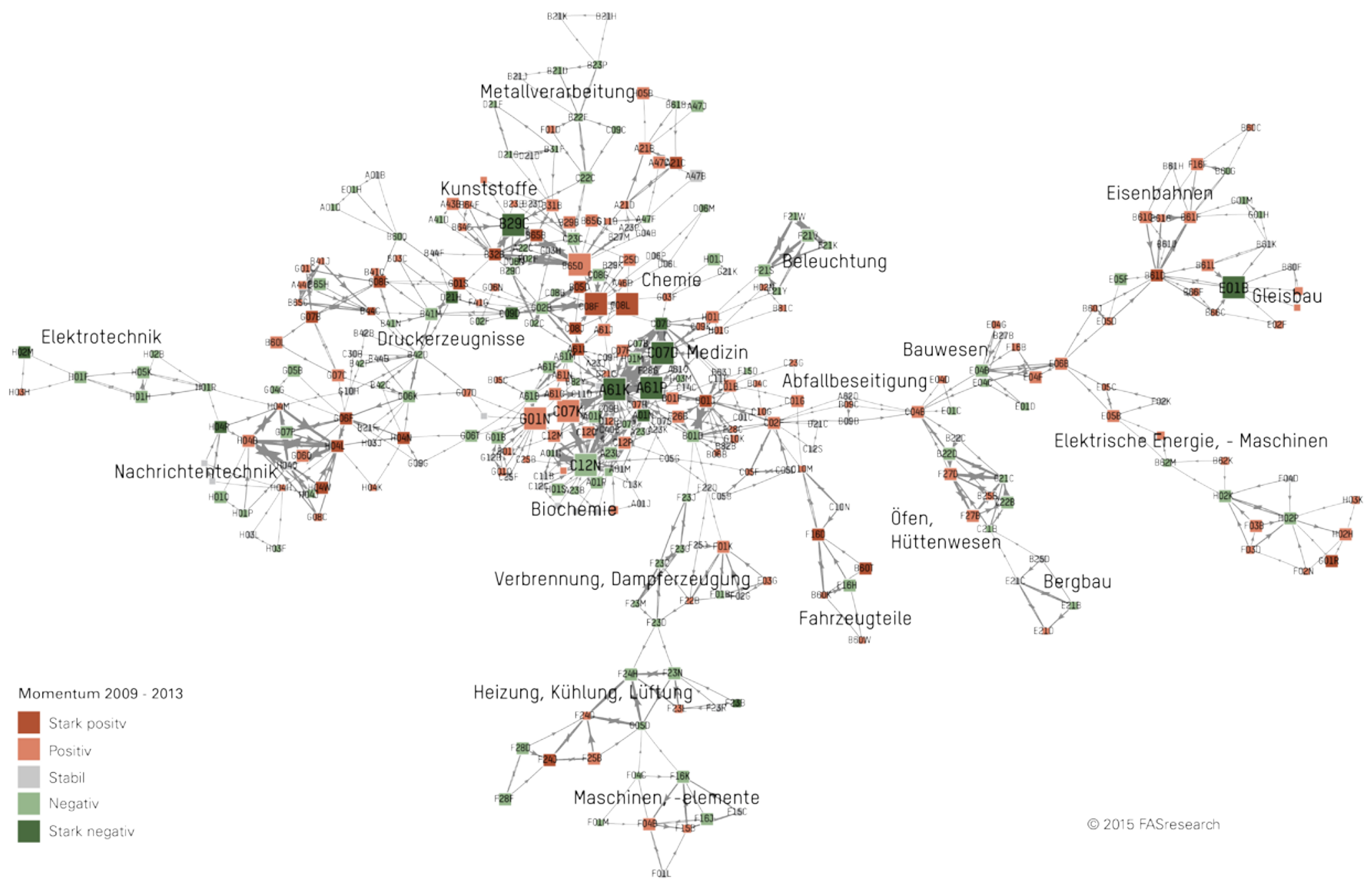




\section{$F A S_{\text {research }}^{\text {inantabibataty }}$}

Datenquelle: Espacenet. Analyse und Grafik: FASresearch. Die Netzwerkknoten stellen die Technologiefelder dar (IPC Patentklassen). Die Pfeile zeigen Patentzitationen zwischen den Technologiefeldern an. Die Farben beziehen sich auf die Entwicklung der Patentzahlen (Zu- oder Abnahme) in den Jahren 2009 bis 2013. 
Mit Hilfe einer netzwerkanalytischen Clustering-Methode (Louvain-Clustering) lassen sich Gruppen von Technologiefeldern identifizieren, innerhalb derer die Netzwerkdichte größer ist als zwischen ihnen (Modularität). In der Abbildung sind diese Cluster auch mit Bezeichnungen versehen, die sich auf die wichtigsten Patentklassen (gemessen an der Anzahl der Patente 2009 bis 2013) beziehen. Im Zentrum des Netzwerks befindet sich ein Cluster, der sich aus humanmedizinischen, biochemischen und biotechnologischen Technologiefeldern zusammensetzt. Innerhalb dieses Clusters weisen die Patentklassen, die sich auf Medikamentenentwicklung und -erzeugung beziehen, eher ein negatives Momentum auf; gewisse Klassen der organischen Chemie hingegen ein positives (z.B. C07K Peptide). Oberhalb dieses Clusters befindet sich eine weitere zentrale Gruppe von Technologiefeldern, die zur chemischen Industrie gehören, und die von Borealis-Patenten dominiert wird. Um dieses (bio)chemische Zentrum herum siedeln sich verschiedene Cluster von Patenklassen an, die teilweise der "klassischen Industrie" zuzurechnen sind und die zum Teil ein durchschnittlich negatives Momentum aufweisen (Heizung, Kühlung; Maschinenbau, Elektrotechnik, Metallverarbeitung, Dampftechnologien) und zum Teil ein durchschnittlich positives (Eisenbahn, Gleisbau; Verkehrssteuerung, -regelung; Kunststoffverarbeitung, Nachrichtentechnik). Das folgende Diagramm fasst die Cluster zu Knoten zusammen und trägt sie in einem Streudiagramm auf, in dem die x-Achse das durchschnittliche Momentum der Patentklassen innerhalb jedes Clusters und die y-Achse die Summe der Patente jedes Clusters (der Anschaulichkeit wegen gerankt) widergibt. 


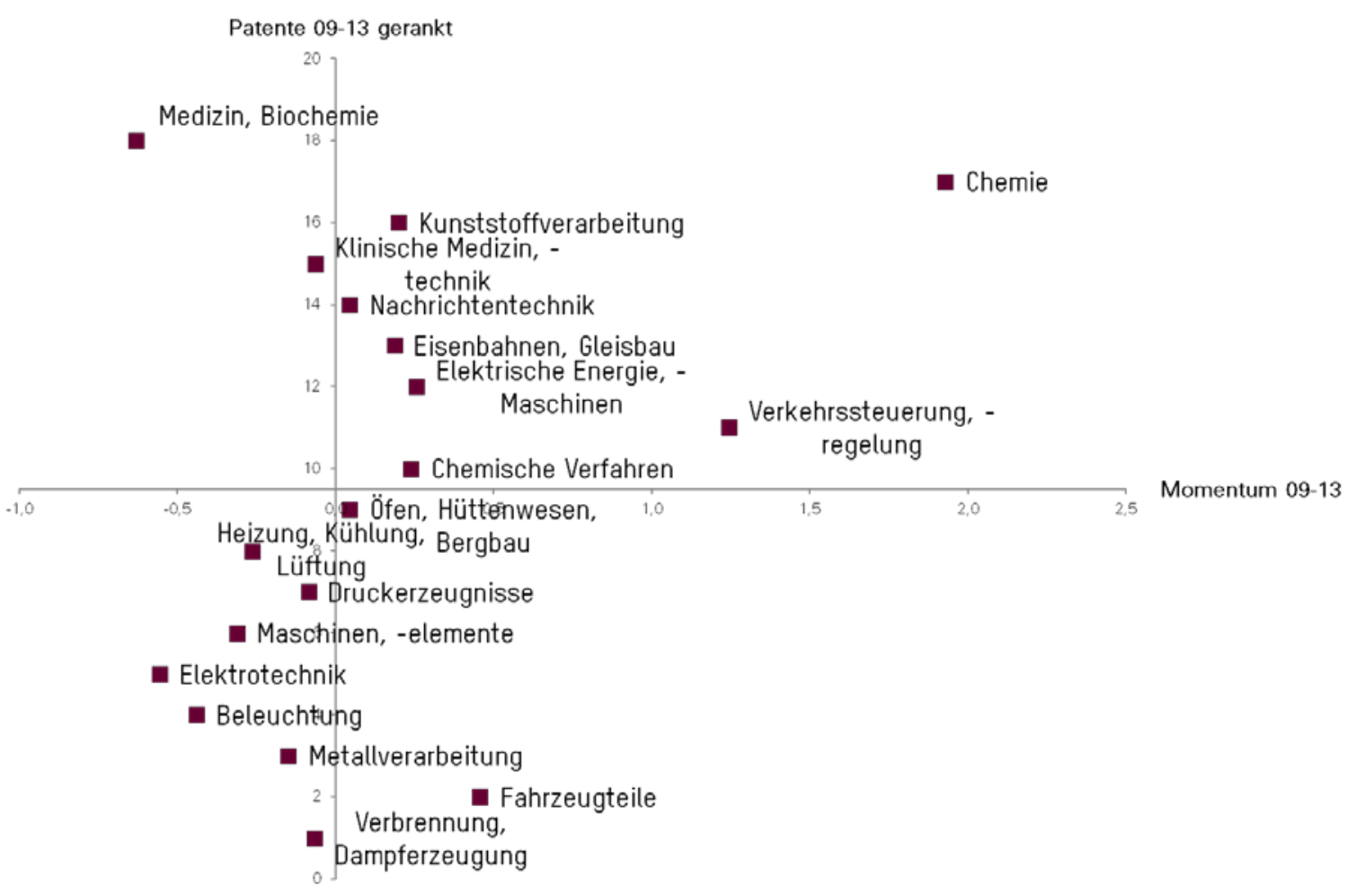

Datenquelle: Espacenet. Analyse: FASresearch

Je weiter oben sich ein Cluster befindet, desto mehr Patente gab es im Zeitraum 2009 2013. Je weiter rechts ein Cluster angesiedelt ist, desto größer ist die durchschnittliche jährlich Zunahme an Patenten im angegebenen Zeitraum. Das bedeutet, im rechten oberen Quadranten des Diagramms befinden sich die Technologiefelder, die aufgrund der Patentzahl bedeutend sind und die darüber hinaus auch wachsen (z.B. Chemie), im linken oberen Quadranten die bedeutenden Technologiefelder, die hinsichtlich der Patentzahlen stagnieren oder schrumpfen (Medizin).

\subsubsection{Zitationsnetzwerk der Anmelder}

Ebenso wie mit den Patentklassen lässt sich aus den Daten auch ein Netzwerk mit den Anmeldern generieren, die durch die Zitationen verbunden sind, die zwischen ihren $\mathrm{Pa}$ tenten bestehen. Die Tabelle 21 zeigt, welche Patentanmelder über Patente verfügen, die besonders häufig von anderen Patenten zitiert werden. Die Spalte "Zitationen" bezieht sich auf die Anzahl der Patente, von denen die Patente des jeweiligen Anmelders zitiert werden. Die Spalte "Patente" meint die Anzahl der Anmeldungen im Zeitraum 1979 bis 
2014. "Zitationen pro Patent" ergibt sich aus der Division der zweiten und dritten Spalte. Es fällt auf, dass in der Liste fast ausschließlich Unternehmen aus dem Life ScienceBereich vertreten sind, einem Feld, das der Sphäre der wissenschaftlichen Exzellenz angehört und in dem deshalb die Zitationen eine größere Rolle spiele dürften.

Tabelle 21 - Wlener Patentanmelder mlt den melsten Zltationen pro Patent

\begin{tabular}{|c|c|c|c|}
\hline Name & Zitationen & Patente & $\begin{array}{l}\text { Zitationen } \\
\text { pro Patent }\end{array}$ \\
\hline INTERCELL AG (VALNEVA) & 640 & 121 & 5,3 \\
\hline NOVARTIS PHARMA GMBH & 6.684 & 1.647 & 4,1 \\
\hline AUSTRIA CARD PLASTIKKARTEN & 42 & 15 & 2,8 \\
\hline BAXTER AKTIENGESELLSCHAFT & 338 & 129 & 2,6 \\
\hline NABRIVA THERAPEUTICS & 43 & 18 & 2,4 \\
\hline POLYMUN SCIENTIFIC IMMUNBIOLOGISCHE FORSCHUNG & 61 & 26 & 2,3 \\
\hline F-STAR & 53 & 26 & 2,0 \\
\hline OMV AKTIENGESELLSCHAFT & 34 & 18 & 1,9 \\
\hline FIBREX MEDICAL RESEARCH \& DEVELOPMENT GMBH & 31 & 17 & 1,8 \\
\hline IGENEON KREBS-IMMUNTHERAPIE & 51 & 28 & 1,8 \\
\hline AFFIRIS FORSCHUNGS- UND ENTWICKLUNGS & 24 & 14 & 1,7 \\
\hline STEYR-DAIMLER-PUCH SPEZIALFAHRZEUG GMBH & 25 & 15 & 1,7 \\
\hline ROBERT BOSCH AG & 28 & 18 & 1,6 \\
\hline AVIR GREEN HILLS BIOTECHNOLOGY & 38 & 25 & 1,5 \\
\hline GEOPHARMA PRODUKTIONSGMBH & 10 & 7 & 1,4 \\
\hline UV \& P UMWELTMANAGEMENT-VERFAHRENSTECHNIK & 7 & 5 & 1,4 \\
\hline SANOCHEMIA PHARMAZEUTIKA AG & 52 & 39 & 1,3 \\
\hline AGRANA STÄRKE GMBH & 9 & 7 & 1,3 \\
\hline NABRIVA THERAPEUTICS AG & 43 & 34 & 1,3 \\
\hline \multicolumn{4}{|l|}{ Datenquelle: Espacenet. Analyse: FASresearch } \\
\hline \multicolumn{4}{|c|}{ Welche Anmelder sind nun miteinander durch Zitationsbeziehungen verbunden? Die } \\
\hline \multicolumn{4}{|c|}{ nächste Abbildung (Abb. 22) stellt das Netzwerk der Patentzitationen zwischen Wiener } \\
\hline \multicolumn{4}{|c|}{ und internationalen Anmeldern in den Jahren 2004 bis 2014 dar. Die letzten zehn Jahre } \\
\hline
\end{tabular}

Insgesamt liegen uns für den genannten Zeitraum 5.150 Wiener Patente vor. Die Datengrundlage für das Zitationsnetzwerk bilden 6.069 Patente, die aufeinander Bezug nehmen; davon 2.119 aus Wien. Das heißt, 41.1\% der Wiener Patente (2.119 von 5.150) sind in 
Zitationsbeziehungen eingebettet. Die Netzwerkknoten stellen die Akteure dar; jene aus Wien sind orange gekennzeichnet. Ein Pfeil zwischen zwei Akteuren bedeutet, dass der eine Akteur mindestens ein Patent des anderen im Untersuchungszeitraum zitiert hat.

Es lassen sich mehrere Subzentren erkennen, vor allem aber gibt es eine linke Hälfte, in der die produzierende Industrie vorherrscht (Infrastruktur, Verkehr, Transport, Nachrichtentechnik, IKT) und in der Unternehmen wie Siemens, Kapsch, Telekom Austria, T-Mobile Austria, TTTECH etc. angesiedelt sind, und eine rechte, die durch die Life Sciences (Valneva, F-Star, Affiris, Biomay usw.) sowie durch die chemische Industrie (Borealis rechts oben) geprägt ist. Interessant ist die Position einiger Subzentren. Der Cluster mit den Schienenbahnen (ÖBB) links oben ist durchaus mit dem Industriecluster verbunden, befindet sich aber an dessen Peripherie und ist durch eine Brücke, die von der Semperit AG Holding (Medizintechnologie) gebildet wird, an die Life Science-Hälfte gekoppelt. Der Cluster, in dessen Zentrum sich die Haas Food Equipment befindet, der wir schon im Zusammenhang mit den Technologiefeldern mit positivem Momentum begegnet sind, findet sich hingegen auf der Seite der Life Sciences, die zum Bereich der Lebensmittelproduktion adjazent sind. Und auch die Borealis, einer der Schlüsselspieler bei den Patenten Wiens in den letzten Jahren, bildet einen Subcluster an der Peripherie des Netzwerks.

Die beiden Hälften (Infrastruktur, Verkehr, IKT links sowie die Life Sciences rechts) stellen sich als die Zentren des Netzwerks dar und repräsentieren damit die Stärkefelder Wiens im Hinblick auf die Patentierungen in den letzten zehn Jahren. Und sie sind auch miteinander verbunden - in der Mitte der Karte sind die Akteure angesiedelt, die diese Verbindungen herstellen. Dass die Medizintechnik sehr gut dazu geeignet ist, die Life Sciences und verschiedene Technikbereiche miteinander zu verknüpfen, haben wir bereits am Beispiel Semperit AG Holding gesehen. Die Otto Bock Healthcare Products $\mathrm{GmbH}$, die sich etwas oberhalb der Mitte der Karte befindet, ist noch besser vernetzt und nimmt diese Broker-Position in noch höherem Maße ein. Außerdem sind es die Institutionen der 
außeruniversitären Forschung, allen voran das $\mathrm{AIT}^{26}$, aber auch die Akademie der Wissenschaften, die aufgrund der Heterogenität ihrer Aktivitäten eine Brückenfunktion zwischen den beiden zentralen Patentbereichen Wiens ausüben. Auf die Universitäten hingegen scheint dies überraschenderweise weniger zuzutreffen; sie befinden sich allesamt (neben der Uni Wien, der BOKU und der Vetmed vor allem auch die TU!) in der rechten Hälfte der Patentlandkarte Wiens, das heißt im Bereich der Life Sciences.

${ }^{26}$ Die Austrian Research Centers (ARC), die Vorgängerorganisation des AIT, die im Untersuchungszeitraum ebenfalls noch aktiv war, wurde in der Karte als eigenständiger Netzwerkknoten belassen, um die diachrone Dimension zu bewahren, die durch die Synchronizität dieser Netzwerkkarten die vorliegende bildet immerhin einen Zeitraum von zehn Jahren ab - unter den Tisch zu fallen droht. Das gleiche gilt für Unternehmen, die es heute nicht mehr gibt (Avir Green Hills) oder die sich mit anderen Firmen verbunden haben und unter einem anderen Namen wieder aufscheinen (Intercell, Valneva). 
Abb. 24 - Netzwerk der Patentzltationen zwlschen Wlener und Internationalen Anmeldern 2004 -2014

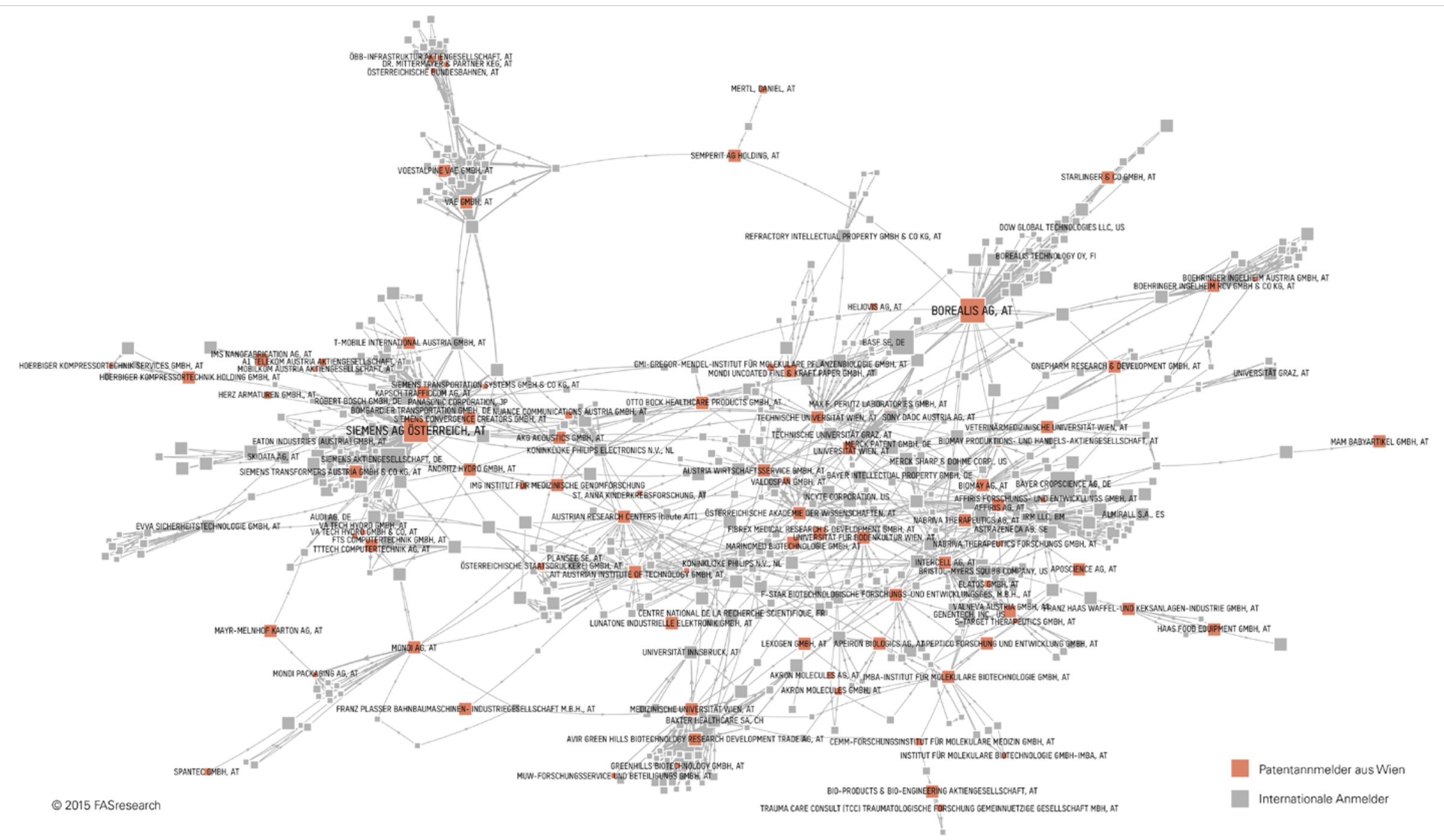




\section{FAS research}

Datenquelle: Espacenet. Analyse und Grafik: FASresearch. Die Pfeile zeigen die Zitationen zwischen den Patenten der jeweiligen Unternehmen an. Die Größe der Unternehmen bezieht sich auf die Anzahl der Unternehmen, von denen sie im bestehenden Netzwerk zitiert werden. 
Wir sehen auch, dass das Netzwerk, wenn wir die internationalen Anmelder aus der Karte entfernen und nur die Wiener Akteure belassen würden, in Fragmente zerfallen würde, die untereinander nicht miteinander verbunden sind. Dies soll keineswegs als Mangel hervorgehoben werden, im Gegenteil, es zeigt, wie sehr die Wissens- und Technologieproduktion international eingebettet ist. Die folgende Tabelle zeigt, auf welche Firmen sich Wiener Akteure besonders häufig beziehen, wenn sie ihre Patente anmelden (Backward Citation, Input an Know-how): ${ }^{27}$

Tabelle 22 - Wen zitieren Wiener Patente - Backward Citation, Input an Know-how nach Wien 2004 - 2014.

\begin{tabular}{lcc} 
Anmelder & Zitationen & Prozent \\
\hline BOREALIS TECHNOLOGY OY, FI & 1107 & $23,1 \%$ \\
NOVARTIS AG, CH & 592 & $12,4 \%$ \\
BASELL POLIOLEFINE ITALIA S.R.L., IT & 76 & $1,6 \%$ \\
BOREALIS POLYMERS OY, FI & 74 & $1,5 \%$ \\
BASELL POLYOLEFINE GMBH, DE & 64 & $1,3 \%$ \\
DOW GLOBAL TECHNOLOGIES INC., US & 55 & $1,1 \%$ \\
EXXONMOBIL CHEMICAL PATENTS INC., US & 46 & $1,0 \%$ \\
DOW GLOBAL TECHNOLOGIES LLC, US & 46 & $1,0 \%$ \\
XENON PHARMACEUTICALS INC., CA & 40 & $0,8 \%$ \\
GLAXO GROUP LIMITED, GB & 37 & $0,8 \%$ \\
ALTANA PHARMA AG, DE & 37 & $0,8 \%$ \\
SIEMENS AKTIENGESELLSCHAFT, DE & 30 & $0,6 \%$ \\
BAXTER HEALTHCARE SA, CH & 28 & $0,6 \%$ \\
ASTRAZENECA AB, SE & 27 & $0,6 \%$ \\
MERCK SHARP \& DOHME CORP., US & 24 & $0,5 \%$ \\
NYCOMED GMBH, DE & 24 & $0,5 \%$ \\
IRM LLC, BM & 23 & $0,5 \%$ \\
LUMMUS NOVOLEN TECHNOLOGY GMBH, DE & 22 & $0,5 \%$ \\
CIBA HOLDING INC., CH & 21 & $0,4 \%$ \\
MERCK \& CO., INC., US & 20 & $0,4 \%$
\end{tabular}

Datenquelle: Espacenet. Analyse: FASresearch

Die Zitationsbeziehungen sind natürlich durch die großen Player geprägt, und wir sehen, dass die Zitationen oftmals innerhalb der Konzerne und zwischen Mütter-/Töchterfirmen verlaufen (Boralis, Novartis). Die stärksten Verbindungen bestehen in den Life Sciences (Bsp. Novartis), der chemischen Industrie (Borealis) und in technisch ausgerichteten Berei-

\footnotetext{
${ }^{27}$ Die Akteure in Tabelle 22 und in Tabelle 23 sind auch in der Netzwerkkarte (Abb. 24) enthalten, aus Gründen der Darstellbarkeit sind jedoch die Beschriftungen ausgeblendet.
} 
chen (Siemens). 25,2\% aller Backward Citations aus Wien gingen nach Finnland, 21\% in die USA, $16,9 \%$ in die Schweiz, 11,2\% nach Deutschland und 3,5\% nach Japan. 
Tabelle 23 - Wer zitiert Wiener Patente - Fonward Citation, Output an Know-how aus Wien 2004 - 2014

\begin{tabular}{lcc} 
Anmelder & Patente & Prozente \\
\hline NOVARTIS AG, CH & 1325 & $28,9 \%$ \\
BOEHRINGER INGELHEIM INTERNATIONAL GMBH, DE & 104 & $2,3 \%$ \\
INCYTE CORPORATION, US & 85 & $1,9 \%$ \\
IRM LLC, BM & 49 & $1,1 \%$ \\
ASTRAZENECA AB, SE & 48 & $1,0 \%$ \\
NOVARTIS PHARMA AG, CH & 39 & $0,9 \%$ \\
SANOFI, FR & 36 & $0,8 \%$ \\
MERCK SHARP \& DOHME CORP., US & 32 & $0,7 \%$ \\
SANDOZ AG, CH & 31 & $0,7 \%$ \\
KYORIN PHARMACEUTICAL CO., LTD., JP & 30 & $0,7 \%$ \\
THERAVANCE, INC., US & 30 & $0,7 \%$ \\
SIEMENS AKTIENGESELLSCHAFT, DE & 27 & $0,6 \%$ \\
ALMIRALL S.A., ES & 27 & $0,6 \%$ \\
MERCK PATENT GMBH, DE & 23 & $0,5 \%$ \\
SANOFI-AVENTIS, FR & 23 & $0,5 \%$ \\
ASTELLAS PHARMA INC., JP & 22 & $0,5 \%$ \\
SCHERING CORPORATION, US & 22 & $0,5 \%$ \\
GENENTECH, INC., US & 21 & $0,5 \%$ \\
TEVA PHARMACEUTICAL INDUSTRIES LTD., IL & 19 & $0,4 \%$ \\
SPEEDEL EXPERIMENTA AG, CH & 19 & $0,4 \%$ \\
& &
\end{tabular}

Datenquelle: Espacenet. Analyse: FASresearch

\subsection{Das Patentportfolio Wiens}

Ans Ende des Abschnitts über die Patente stellen wir die Darstellung des Wiener Patentportfolios in Form von Treemaps (siehe die folgenden beiden Seiten). Die Abb. 25 zeigt die Technologiefelder (IPC auf der Ebene der Klassen) nach der Anzahl der Patente seit 1979 sowie nach deren Durchschnittsalter. Die Klassen sind entsprechend ihrer Zugehörigkeit zu den Sektionen A bis $G$ angeordnet. Die Flächengröße bezieht sich auf die Anzahl der Patente und die Farbe auf das Durchschnittsalter (je dunkler, desto jünger die jeweilige Patentklasse. Bei der Abb. 26 bezieht sich die Flächengröße auf die Anzahl der Patente 2009 bis 2013 und die Farbe auf das Momentum (je dunkler, desto größer die durchschnittliche Zunahme an Patenten im gleichen Zeitraum). Die erste Treemap bildet 
somit das Patentportfolio Wiens ab, soweit uns die Daten in digitalisierter Form vorliegen. Die zweite zielt auf die Entwicklungen in jüngerer Zeit ab. 


\section{Abb. 25 - Patentportfolio Wiens: IPC-Klassen nach der Anzahl der Wiener Patente 1979 - 2014 und nach dem Durchschnittsalter der Patente}

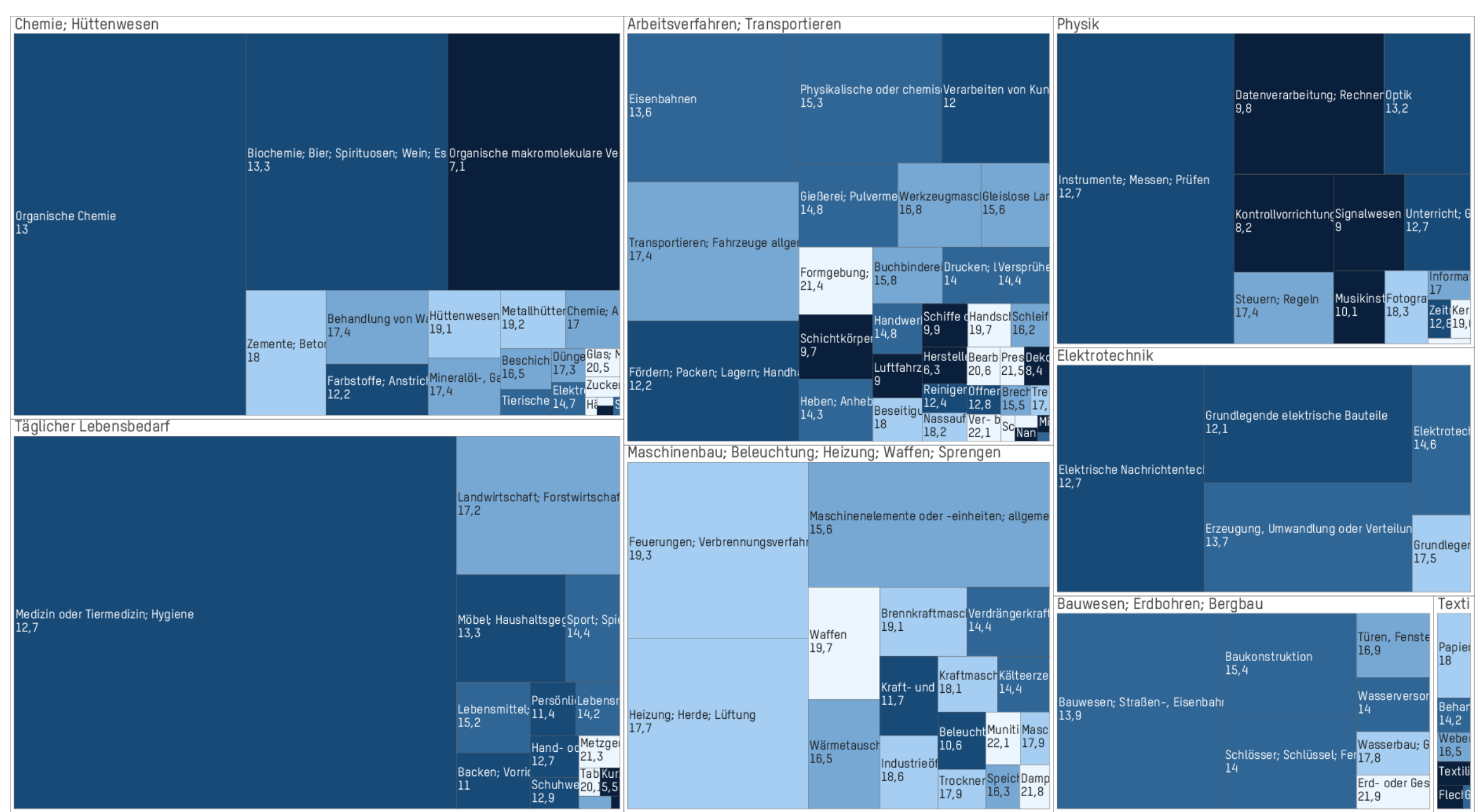

Datenquelle: Depatisnet, Espacenet. N = 16.906 Patente. Analyse: FASresearch. Flächengröße: Anzahl der Wiener Patente in der jeweiligen Technologieklasse 1979 - 2014. Farbe: Durchschnittsalter der Patente innerhalb der jeweiligen Technologieklasse (je dunkler, desto jünger). 
Abb. 26 - Patentportfollo Wiens: IPC-Klassen nach der Anzahl der Wiener Patente 2009 - 2013 und nach dem Momentum 2009-2013.

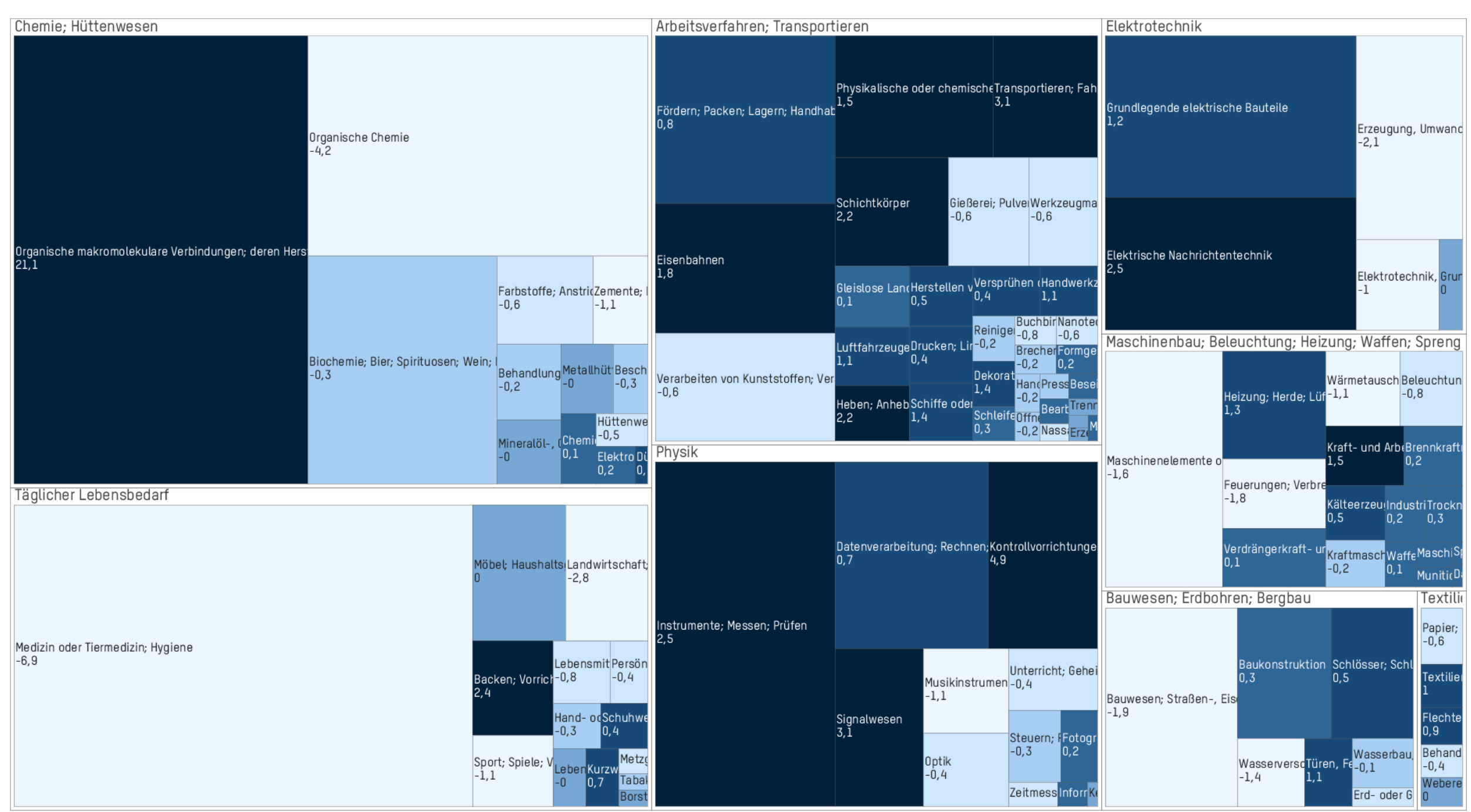




\section{FAS research}

Datenquelle: Depatisnet, Espacenet. N = 16.906 Patente. Analyse: FASresearch. Flächengröße: Anzahl der Wiener Patente in der jeweiligen Technologieklasse 2009 - 2013. Farbe: Momentum 2009 - 2013 (je dunkler, desto stärker die Zunahme der Patentzahl in diesem Zeitraum). 
Ein Beispiel zu Abb. 25: Die größte Sektion gemessen an der Zahl der Wiener Patente 1979 - 2014 stellt C-Chemie; Hüttenwesen dar, darum ist sie durch die größte Fläche links oben dargestellt. Innerhalb dieser Sektion ist gemessen an der Patentzahl die Klasse C07-Organische Chemie am größten. Die Klasse mit dem geringsten Durchschnittsalter der Patente ist C08-Organische makromolekulare Verbindungen (7,1 Jahre) und dementsprechend durch eine dunklere Fläche dargestellt. Ein Beispiel zu Abb. 26: Die Flächengrößen beziehen sich hier auf die Zahl der Wiener Patente im Zeitraum 2009 - 2013. Innerhalb der Sektion Chemie weist die Klasse C08-Organische makromolekulare Verbindungen sowohl die größte Patentzahl als auch die größte durchschnittliche jährlich Zunahme an Patenten auf (größte und dunkelste Fläche).

Zusammenfassung Wiener Patentlandschaft:

- Die Transition der Wiener Wirtschaftsstruktur von der "Old Economy" (Verbrennungsverfahren, Bauwesen, Elektronik) hin zu IKT, Life Sciences und Smart Production ist deutlich in der Veränderung des Patentportfolios der Stadt abgebildet. Das PatentZitationsnetzwerk besteht aus den beiden Polen IKT/Nerkehrs-/Nachrichtentechnik (Siemens, Kapsch, TTTECH) und Life Sciences (Affiris, Biomay und Valneva) vermittelt durch die Medizintechnik (z.B. Otto Bock) sowie insbesondere der außeruniversitären Forschung (AIT). Auffallend ist die eher periphere Einbettung der "traditionellen" Industrie (Borealis, Mondi, Semperit und ÖBB) in der Wiener Patentlandschaft, was vor allem vor dem Hintergrund einer Wiener Industrie 4.0-Strategie zu beachten wäre.

- Auffällige Distanz der TU Wien von den eigentlichen Motoren der IKT (Siemens, Kapsch, TTTECH) hinsichtlich der Patentzitationen, die TU hat hier sogar eine größere Nähe zum Feld der Life Sciences als zum Zentrum der IKT. Die Patentierungen der TU dürften demnach eher in den Bereichen der technischen Chemie bzw. der technischen Biowissenschaften angesiedelt sein (die wichtigsten Patentfelder auf der Ebene der IPC-Unterklasse sind: G01N-Untersuchen oder Analysieren von Stoffen durch Bestim- 
men ihrer chemischen oder physikalischen Eigenschaften; C12N-Mikroorganismen oder Enzyme; H01S-Vorrichtungen, die stimulierte Emission verwenden).

- Deutliche Technologie-Zyklus-bedingte Sättigungseffekte in den Patentklassen feststellbar; z.B. im Bereich Medizin sehen wir eine kontinuierliche Abnahme von Patenten (vor allem auch begründet durch die starke Abnahme der Patentierungen von Novartis), ähnliches im Bauwesen, Maschinen etc.

- Nichtsdestotrotz stellt der Life Science-Bereich nach wie vor ein wichtiges Technologiefeld auch bei den Patenten dar, weil er - anders als etwa der von einem Player dominierte Bereich der Chemie - eine Vielzahl an kleinen bis mittelgroßen, nach wie vor aktiven Patentanmeldern versammelt. Darüber hinaus bildet er jenen Bereich, über den die Wiener Patentlandschaft am meisten durch Zitationen in die internationale Patentlandschaft eingebettet ist.

- Stärkstes Momentum in den jungen Patentbereichen in den Feldern Drahtlose Kommunikationsnetze, digitale Datenverarbeitung, Verkehrstechnologien, Biozide, Lichtquellen und Nanotechnik, aber auch in den eher "traditionellen" Bereichen der Fahrkartenausgeber, Backen-Backöfen sowie Wassergewinnung-und Aufbereitung.

\section{Wiener Kreatlvwirtschaft und Soclal Entrepreneurship}

Der missions- und problemlösungsorientierte Bereich der Wiener Innovationslandschaft war nicht der zentrale Gegenstand dieser Studie. Aus der Sicht des von uns vertretenen innovationspolitischen Konzepts wäre es jedoch sinnvoll, die Sphäre der Mission ebenso wie die der wissenschaftlichen Exzellenz und des ökonomischen Nutzens in zukünftige Innovationsstrategien zu integrieren und miteinander in Verbindung zu bringen - darum hier in einem kurzen Abschnitt einige zentralen Eckpunkte der Entwicklung des missionsorientierten Bereichs anhand der Beispiele Kreativwirtschaft und Social Entrepreneurship. 
Typisch für den missionsorientierten Innovationsbereich ist grundsätzlich, dass er weniger eindeutig definiert und von den anderen Bereichen abgegrenzt werden kann. Dies gilt auch für die Währungen, die im missionsorientierten Bereich gelten. Wissenschaftliche Exzellenz oder ökonomischer Nutzen lassen sich - wie problematisch auch immer - viel leichter messen (Anzahl der Publikationen, lukrierte Forschungsgelder, Umsatz und Gewinn) als die Erreichung der missionsbezogenen Ziele - denn was bedeuten "social impact" oder "responsible science"? Welche Ziele verfolgt eine Designerin mit ihrer Tätigkeit über die bloße Exiszenzsicherung hinaus? Nach welchen gesellschaftspolitischen oder ästhetischen Kriterien könnte das beurteilt werden? - Diese Schwierigkeiten bei der Skalierung stellen sich zunächst einmal als Mangel dar, denn eine soziale Gruppe hat desto mehr Einfluss, je besser und leichter die Mitglieder ihre Zugehörigkeit definieren und ihren Status innerhalb der Gruppe messen können. Gerade diese Ambiguität und die Heterogenität der Akteure könnten jedoch von Vorteil sein, wenn es darum geht, die verschiedenen Innovationsbereiche miteinander zu verbinden. Sie machen das Wesen der missionsorientierten Bereiche aus.

Der sechste Kreativwirtschaftsbericht der Wirtschaftskammer Österreich definiert Kreativwirtschaft folgendermaßen: „Die Kreativwirtschaft (umfasst) all jene erwerbsorientierten Unternehmen, die sich mit der Schaffung, Produktion und (medialen) Distribution von kreativen und kulturellen Gütern und Dienstleistungen beschäftigen. (...) Die unterschiedlichen Bereiche, die der Kreativwirtschaft zugeordnet werden, sind Architektur, Design, Musik, Buch und künstlerische Tätigkeit, Radio und TV, Software und Games, Verlage, Video und Film und Werbung." ${ }^{28}$ Social Entrepreneurship bezeichnet das soziale Unternehmertum, dessen Tätigkeiten sich auf die Lösung gesellschaftlicher Probleme bzw. auf die po-

\footnotetext{
${ }^{28}$ Creativ Wirtschaft Austria: Sechster österreichischer Kreativwirtschaftsbericht, S. 27. Download unter http://www.creativwirtschaft.at/document/6.KWB_Langfassung_WEB.pdf, abgerufen am 16.07.2015.
} 
sitive Veränderung von Gesellschaft beziehen. ${ }^{29}$ Auf die Aspekte der Kreativität bzw. der Lösung gesellschaftlicher Probleme rekurriert die Zuordnung der Kreativwirtschaft und des Social Entrepreneurship zur missionsorientierten Innovationssphäre.

- Wir stellen ein überproportionales Wachstum der Kreativbranchen in Wien fest, was in der deutlichen Zunahme des Anteils der Wiener Kreativwirtschaft am Gesamtbestand der kreativen Unternehmungen in Österreich zum Ausdruck kommt (von 45\% auf 54\% zwischen 2009 und 2014). Wien ist das Zentrum der Kreativwirtschaft Österreichs. ${ }^{30}$

- Verdichtete Vernetzungsprofile in den Bereichen Design, Musik, Mode sowie Multimedia/Software/Games mit hoher interner Diversität an Akteuren aus den wirtschaftlichen, (außer-) universitären und zivilgesellschaftlichen Bereichen. Allerdings dominieren EPUs und KMUs; große, traditionelle (Industrie)Unternehmen fehlen fast vollständig.

- Dichte Community-Netzwerke bestehen bei den Social Entrepreneurs und "Change Makern" in den Feldern Bildung/Kinder/Jugend, Ökologie/Energie/Regionalentwicklung, Migration sowie Gesundheit. ${ }^{31}$

- Relativ hoher Fragmentierungsgrad der Social Entrepreneurs im Feld Bildung/Integration; signifikante strukturelle Löcher zwischen den Themenfeldern Gesundheit und Integration, sowie Gesundheit und Bildung sowie sehr auffällig zwischen engagierten AkteurInnen im Feld Bildung und jenem im Bereich der (neuen) Energieund Mobilitätsformen. ${ }^{32}$ Dies ist typisch für "junge" gesellschaftliche Bereiche: Es gibt

${ }^{29}$ Definition nach Gabler Wirtschaftslexikon, http://wirtschaftslexikon.gabler.de/Archiv/152065/social-entrepreneurship-v5.html, abgerufen am 16.07.2015.

30 Vgl. dazu auch den Sechsten österreichischen Kreativwirtschaftsbericht, S. 28 (Entwicklung der Unternehmenszahlen in den letzten Jahren) und S. 52 (Bedeutung Wiens).

31 Beispiele für Social Entrepreneurs im Bildungsbereich: Watchado GmbH, TeachForAustria gGmbH, familylab Österreich. Im den Bereichen Ökologie/Energie/Regionalentwicklung: Pioneers of Change, die umweltberatung, Atlans $\mathrm{GmbH}$.

32 FASresearch: Changemaker Map 2013, unveröffentlichte Studie. 
eine Vielzahl an sehr unterschiedlichen Akteuren, zwischen denen nur wenige Verbindungen bestehen. Erst mit der Zeit steigt auch der Vernetzungsgrad innerhalb der und zwischen den Bereichen.

- Die einzelnen Bereiche der Kreativwirtschaft sowie der Wiener Social Entrepreneurs sind durch signifikante Gender-Unterschiede gekennzeichnet, so sind im Bereich Energie/Ökologie 72\% der Social Enrepreneurs Männer, im Feld Migration/Integration sind es $63 \%$ Frauen.

\section{Gesamtschau über die untersuchten Innovationsbereiche}

\section{1. Überblick}

Wenn wir alle Unternehmen und Institutionen unseres Datensatzes, die in den untersuchten Innovationsbereichen (FWF, WWTF, EU-Projekte, FFG, Wirtschaftsagentur, Patente, Kreativwirtschaft und Social Entrepreneurship) im Zeitraum 2009 - 2013 vorkommen, zusammenfassen, gelangen wir zu folgender Aufstellung: ${ }^{33}$

Tabelle 24 - Dle Akteure der untersuchten Innovatlonsberelche 2009 - 2013 nach Kategorlen

\begin{tabular}{lcc} 
Kategorie & Anzahl & Prozent \\
\hline Unternehmen & 1.949 & $68,0 \%$ \\
Einzelpersonen & 450 & $15,7 \%$ \\
Zivilgesellschaft & 188 & $6,6 \%$ \\
Außeruniversitäre Forschung & 106 & $3,7 \%$
\end{tabular}

${ }^{33}$ Es handelt sich nicht um eine repräsentative Stichprobe (im Sinne einer gleichen oder angebbaren Chance für alle Wiener Unternehmen oder Organisationen, darin enthalten zu sein), sondern um jene Akteure, die in den in dieser Studie untersuchten Förderinstrumenten und Innovationsbereichen im genannten Zeitraum laut den uns vorliegenden Daten vorkommen. 


\begin{tabular}{lcc} 
Politik, Verwaltung & 91 & $3,2 \%$ \\
Kunst- und Kultureinrichtungen & 67 & $2,3 \%$ \\
Universitäten bzw. Institute & 16 & $0,6 \%$ \\
\hline Gesamt & 2.867 & $100,0 \%$
\end{tabular}

Datenquellen: FWF, WWTF, CORDIS, FFG, Wirtschaftsagentur, Depatisnet, Espacenet, FASresearchNetzwerkmonitor.

Den größten Teil (68\%) machen Unternehmen aus, gefolgt von Einzelpersonen (vor allem bei den Patentanmeldern, aber auch in der Kreativwirtschaft und im Bereich der Social Entrepreneurs), der Zivilgesellschaft (6,6\%; NGOs, Vereine etc.), der außeruniversitären Forschung (106 bzw. 3,7\%), Politik und Verwaltung (91 bzw. 3,2\%), Kunst und Kultur (67; 2,3\%) sowie 16 Universitäten (0,6\%). - Sehen wir uns an, wie sich diese Akteure auf die verschiedenen Bereiche und Förderinstrumente verteilen:

Tabelle 25 - Vertellung der Akteure auf die Bereiche (absolute Zahlen, Mehrfachnennungen möglich)

\begin{tabular}{|c|c|c|c|c|c|c|c|c|c|}
\hline Kategorie & FWF & WWTF & EU & FFG & Agentur & Patente & Kreativ & $\begin{array}{c}\text { Social } \\
\text { E. }\end{array}$ & Gesamt \\
\hline Außeruniversitäre Forschung & 38 & 14 & 56 & 7 & 6 & 18 & 8 & 10 & 106 \\
\hline $\begin{array}{l}\text { Kunst- u. Kultureinrichtun- } \\
\text { gen }\end{array}$ & 10 & 5 & 8 & 5 & 4 & 0 & 45 & 6 & 67 \\
\hline Einzelpersonen & 0 & 1 & 3 & 67 & 5 & 306 & 62 & 6 & 450 \\
\hline Politik, Verwaltung & 8 & 2 & 37 & 10 & 2 & 7 & 32 & 26 & 91 \\
\hline Universitäten bzw. Institute & 12 & 9 & 10 & 0 & 5 & 5 & 8 & 7 & 16 \\
\hline Unternehmen & 3 & 4 & 169 & 879 & 396 & 393 & 396 & 102 & 1949 \\
\hline Zivilgesellschaft & 8 & 4 & 35 & 12 & 14 & 2 & 35 & 102 & 188 \\
\hline Gesamt & 79 & 39 & 318 & 980 & 432 & 731 & 586 & 259 & 2867 \\
\hline
\end{tabular}

Datenquellen: FWF, WWTF, CORDIS, FFG, Wirtschaftsagentur, Depatisnet, Espacenet, FASresearchNetzwerkmonitor. Analyse: FASresearch

Tabelle 26 - Vertellung der Akteure auf dle Berelche (Spaltenprozent, Mehrfachnennungen mögllch)

\begin{tabular}{lccccccccc} 
Kategorie & FWF & WWTF & EU & FFG & Agentur & Patente & Kreativ & $\begin{array}{c}\text { Social } \\
\text { E. }\end{array}$ & Gesamt \\
\hline Außeruniversitäre Forschung & $48,1 \%$ & $35,9 \%$ & $17,6 \%$ & $0,7 \%$ & $1,4 \%$ & $2,5 \%$ & $1,4 \%$ & $3,9 \%$ & $3,7 \%$ \\
Kunst- und Kultureinricht. & $12,7 \%$ & $12,8 \%$ & $2,5 \%$ & $0,5 \%$ & $0,9 \%$ & $0,0 \%$ & $7,7 \%$ & $2,3 \%$ & $2,3 \%$ \\
Person & $0,0 \%$ & $2,6 \%$ & $0,9 \%$ & $6,8 \%$ & $1,2 \%$ & $41,9 \%$ & $10,6 \%$ & $2,3 \%$ & $15,7 \%$ \\
Politik, Verwaltung & $10,1 \%$ & $5,1 \%$ & $11,6 \%$ & $1,0 \%$ & $0,5 \%$ & $1,0 \%$ & $5,5 \%$ & $10,0 \%$ & $3,2 \%$ \\
Universitäten bzw. Institute & $15,2 \%$ & $23,1 \%$ & $3,1 \%$ & $0,0 \%$ & $1,2 \%$ & $0,7 \%$ & $1,4 \%$ & $2,7 \%$ & $0,6 \%$ \\
Unternehmen & $3,8 \%$ & $10,3 \%$ & $53,1 \%$ & $89,7 \%$ & $91,7 \%$ & $53,8 \%$ & $67,6 \%$ & $39,4 \%$ & $68,0 \%$ \\
Zivilgesellschaft & $10,1 \%$ & $10,3 \%$ & $11,0 \%$ & $1,2 \%$ & $3,2 \%$ & $0,3 \%$ & $6,0 \%$ & $39,4 \%$ & $6,6 \%$ \\
\hline Gesamt & $100,0 \%$ & $100,0 \%$ & $100,0 \%$ & $100,0 \%$ & $100,0 \%$ & $100,0 \%$ & $100,0 \%$ & $100,0 \%$ & $100,0 \%$
\end{tabular}


Datenquellen: FWF, WWTF, CORDIS, FFG, Wirtschaftsagentur, Depatisnet, Espacenet, FASresearchNetzwerkmonitor. Analyse: FASresearch

Die Verteilung der Institutionenarten liefert einen Hinweis darauf, welche Währung in welchem Bereich bzw. bei welchem Förderinstrument dominiert. Beim FWF und beim WWTF, bei denen es um die Förderung von Wissenschaft und Forschung geht und die Exzellenz im Vordergrund steht, dominieren Universitäten und außeruniversitäre Forschungseinrichtungen. Bei den EU-Projekten, bei denen es sowohl exzellenz- als auch anwendungsorientierte Förderschienen gibt, sowie bei der FFG, die die Kooperation zwischen Wirtschaft und Forschung fördert, und bei der Wirtschaftsagentur, die in erster Linie Unternehmen adressiert, nimmt der Forschungsanteil ab und der Unternehmensanteil zu. Patente, genuin die Sphäre des ökonomischen Nutzens, werden primär natürlich von Unternehmen und auch von Einzelpersonen angemeldet. Unternehmen und Einzelpersonen dominieren auch in der Kreativwirtschaft. Im Bereich der Social Entrepreneurs (der Lösung gesellschaftlicher Probleme im weitesten Sinne) kommt schließlich die Zivilgesellschaft ins Spiel.

Abb. 27 - Verbindung der Innovationsbereiche durch Wiener Institutionen 2009 -2013

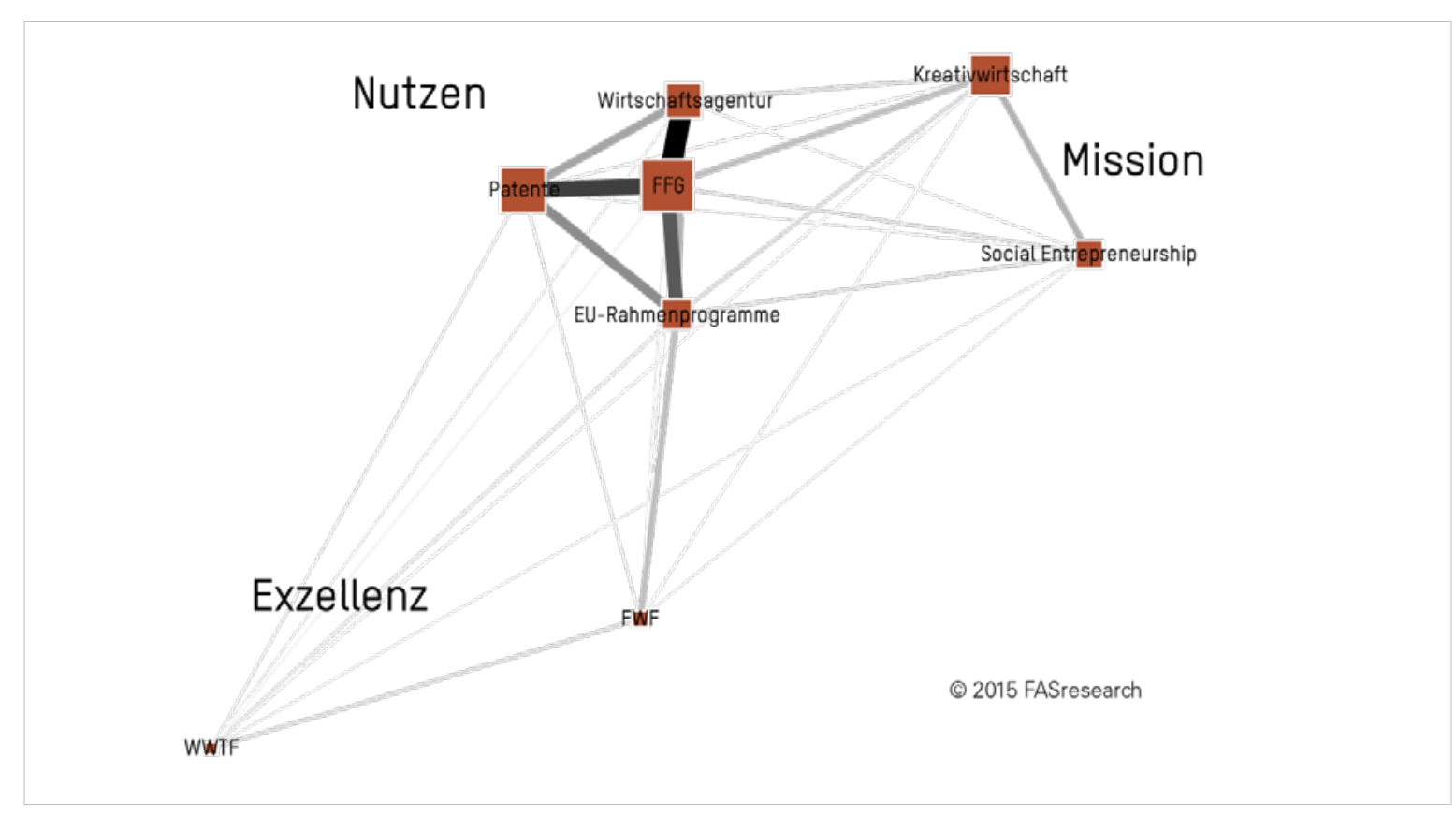

Datenquellen: FWF, WWTF, CORDIS, FFG, Wirtschaftsagentur, Depatisnet, Espacenet, FASresearchNetzwerkmonitor. Analyse und Grafik: FASresearch. Je stärker eine Verbindung, desto größer die Zahl der Institutionen, die jeweils in den beiden Innovationsbereichen vorhanden sind (Schnittmenge der Bereiche). 
Die Abb. 27 stellt in Form eines Netzwerkgraphen dar, wie die verschiedenen Bereiche bzw. Förderinstrumente miteinander durch Akteure verbunden sind. Die Größe der Knoten zeigt die Anzahl der Akteure innerhalb des jeweiligen Bereichs bzw. Instruments an. Eine Linie zwischen zwei Bereichen bedeutet, dass es mindestens einen Akteur (ein Unternehmen oder eine Institution) gibt, der in beiden Bereichen aktiv ist. Je stärker eine Linie, desto mehr gemeinsame Akteure haben die jeweiligen Bereiche.

Die Struktur zeigt, welche Bereiche einander hinsichtlich der Zusammensetzung der Akteure ähneln. Dies gibt auch Hinweise darauf, welche Währungen in einem Bereich vorherrschend sind bzw. zwischen welchen Bereichen es kognitive Distanzen gibt. FWF und WWTF sind exzellenzorientiert und haben viele Akteure gemeinsam. Vom FWF führt der Weg zu den EU-Projekten, die aufgrund ihrer Heterogenität (zum Teil exzellenzbezogen, zum Teil anwendungsorientiert) die Brücke herstellen zu den Förderschienen und Instrumenten, die auf die Kooperation zwischen Wissenschaft und Wirtschaft abzielen (FFG), wirtschaftsnahe Innovation fördern (Wirtschaftsagentur) und schließlich die ökonomische Verwertung betreffen (Patente). Von der FFG und auch von der Wirtschaftsagentur führt der Pfad zur vorrangig missionsorientierten Sphäre von Wirtschaft und Gesellschaft (Kreativwirtschaft, Lösung gesellschaftlicher Probleme).

Eine wichtige, wenn nicht die wichtigste Dimension des innovationspolitischen Konzepts, das dieser Arbeit zugrunde liegt, bezieht sich auf die Empfehlung, die horizontalen Verbindungen zu verstärken, das heißt Beziehungen zwischen ganz unterschiedlichen Bereichen - hier der wissenschaftlichen Exzellenz, dem ökonomischen Nutzen sowie der Problemorientierung - zu ermöglichen, d.h. zwischen solchen, zwischen denen die sozialkognitiven Distanzen besonders groß sind.

Die immer stärkere soziale Differenzierung, der Konkurrenzdruck sowie die Beschränkung der Ressourcen führen in vielen Bereichen dazu, dass sich die sozialen Akteure noch wei- 
ter spezialisieren und sich immer mehr auf das eigene Feld konzentrieren, was einer vertikalen Verstärkung entspricht und eher inkrementelle Innovationen hervorbringt.

Radikale Innovationen hingegen sowie vor allem die Resilienz des gesamten Systems nehmen mit der horizontalen Vernetzung zu. Radikale Innovationen deshalb, weil diese weniger in der Erschaffung von etwas Neuem quasi aus dem Nichts bestehen, sondern vielmehr in der Rekombination und Neuverbindung bestehenden Wissens, das aus unterschiedlichen Bereichen stammt (z.B. die Lösung aus einem Bereich für eine Frage aus einem ganz anderen Bereich). ${ }^{34}$

Und für die Resilienz des Innovationssystems ist horizontale Vernetzung deshalb wichtig, damit dieses den Zyklus aus Entstehung, Wachstum, Destruktion und Erneuerung immer wieder von neuen durchlaufen kann. In jeder dieser Phasen sind unterschiedliche Arten von Akteuren wichtig (siehe Tabelle 1): eher individualistisch orientierte Menschen bei der Entstehung neuer Innovationsbereiche (EinzelforscherInnen, Startups); hierarchisch strukturierte Institutionen in der Phase der Skalierung und des Wachstums (etablierte Unternehmen und Universitäten), "fatalistisch" angelegte Akteure in der Krise (in der es auch darum geht, das Überkommene als solches zu identifizieren und sich von ihm zu trennen) sowie egalitär orientierte in der Phase der Neuorientierung (z.B. Exploration von alternativen Lösungen für gesellschaftliche Probleme, wie das die Social Entrepreneurs für sich beanspruchen). Resilient ist ein System dann, wenn es über alle diese Akteure verfügt und vor allem, wenn diese auch miteinander verbunden sind. Auch dafür bedarf es der horizontalen Vernetzung. ${ }^{35}$

Abb. 28 - Vergleich der beobachteten und der erwarteten Schnittmengen zwischen den Bereichen - Abweichung in Prozentpunkten

\footnotetext{
${ }^{34}$ Rogers, Everett M. (2003).

35 Gunderson, Lance H./Holling C.S. (2002) und Katzmair, Harald (2012) sowie Fath, Brian D./Dean, Carly/Katzmair, Harald (2015).
} 


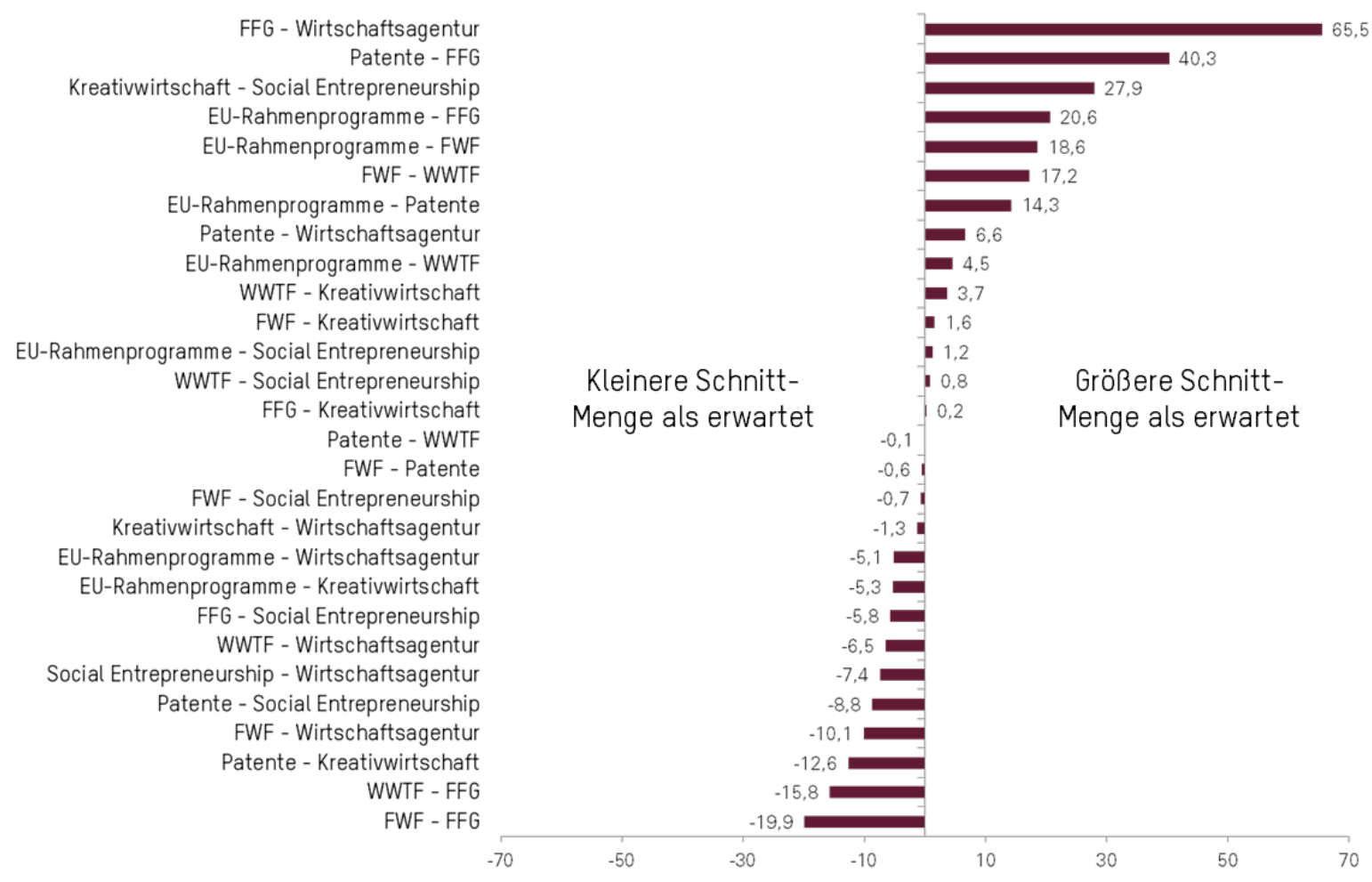

Datenquellen: FWF, WWTF, CORDIS, FFG, Wirtschaftsagentur, Depatisnet, Espacenet, FASresearchNetzwerkmonitor. Analyse: FASresearch

Wir können auf Basis der bestehenden Verbindungen zwischen den Innovationsbereichen (Abb. 27) ermitteln, bei welchen die Schnittmengen größer und bei welchen sie geringer ausfallen, als bei einer Zufallsverteilung zu erwarten gewesen wäre (Abb. 28) $)^{36}$. Wir sehen, dass zwischen FFG und Wirtschaftsagentur sowie zwischen FFG und der Sphäre der Patente die Schnittmenge größer ist als erwartet. Bei der Interpretation ist zu beachten, dass uns für die FFG nur Unternehmensdaten zur Verfügung stehen; wären auch die Universitäten und die außeruniversitären Forschungseinrichtungen enthalten, würden sich auch die Schnittmengen mit WWTF und FWF vergrößern. Doch erscheint das Ergebnis in Bezug auf FFG, Wirtschaftsagentur und Patente (große Schnittmengen an Akteuren) plausibel, weil es bei den drei Bereichen um unternehmensnahe Förderung bzw. um die wirtschaft-

\footnotetext{
${ }^{36}$ Auf Basis der Verteilung der verschiedenen Arten von Akteuren auf die Innovationsbereiche und Förderinstrumente kann berechnet werden, wie stark die Verbindungen zwischen den Bereichen bei einer Zufallsverteilung wären (wenn es keinen Zusammenhang zwischen Bereichen und Akteursarten gäbe). Die Differenz zwischen diesen erwarteten und den beobachteten Verbindungen in Prozentpunkten ist in diesem Balkendiagramm dargestellt.
} 
liche Verwertung geht. Auch dass es zwischen Kreativwirtschaft und Social Entrepreneurship sowie zwischen EU/FFG und EU/FWF eine große Übereinstimmung gibt, ist nicht überraschend. Sehen wir uns an, wo die Schnittmengen unterdurchschnittlich groß sind: zwischen FWF bzw. WWTF und FFG, zwischen den Patenten und der Kreativwirtschaft sowie zwischen FWF und Wirtschaftsagentur. Wir unterschätzen aufgrund der FFG-Daten sicherlich die Verbindungsstärke zu FWF und WWTF, aber dass es zu wenig Übersetzung von der Exzellenz in die Anwendung/Verwertung gibt, haben wir schon im Zusammenhang mit der Feststellung bemerkt, dass der Patentanteil Wiens bei steigender Grundlagenforschung abnimmt. Exzellenz und ökonomischer Nutzen gilt es demnach stärker zu vernetzen. Ähnliches könnte für die Kreativwirtschaft und die Patente gelten. Die Kreativwirtschaft ist in Bereichen angesiedelt, in denen grundsätzlich weniger patentiert wird. Außerdem ist sie in hohem Maße von kleinen Unternehmen (EPUs, KMUs) geprägt, und es gibt in Wien nicht allzu viele Kooperationen mit großen Unternehmen und mit der klassischen Industrie. Die Zusammenarbeit der voestalpine und der Ars Electronica könnte hier ein Vorbild sein; die Kreativwirtschaft verfügt wegen der Diversität ihrer Akteure und vor allem wegen ihrer vielfältigen Aktivitäten, die in mehrerer Hinsicht für die klassische Industrie von Bedeutung sind (IKT, Multimedia, Design), über viele Anschlussmöglichkeiten an die klassische Industrie. - Auch die Links zwischen der Sphäre der Anwendung/Verwertung (Wirtschaftsagentur/Patente) und dem problemlösungsorientierten Bereich (Social Entrepreneurs) sind unterdurchschnittlich ausgeprägt, und auch hier ließen sich viele Möglichkeiten der Verbindungsstärkung entwickeln (siehe unten, Empfehlungen).

Es geht hier nicht darum, willkürlich Verknüpfungsmöglichkeiten zu konstruieren. Nicht bei jeder abwesenden Beziehung (bei jedem strukturellen Loch) ist es sinnvoll und wünschenswert, die Lücke zu schließen. Im Gegenteil: Die (ohnehin nur hypothetische) totale Vernetzung würde zur Vereinheitlichung der Akteure führen; es ist auch die Abwesenheit von Beziehungen, die aus der Sicht der Netzwerktheorie zu Diversität und sozialer Dynamik führt. Eine zu starke Spezialisierung, ein zu hohes Maß an Konzentration auf den ei- 
genen Bereich aber führen dazu, dass es überhaupt keinen Transfer von unterschiedlichen Ressourcen mehr gibt. Es existieren nun einmal unterschiedliche Antriebe für soziale Akteure, Innovationen hervorzubringen (das Streben nach wissenschaftlicher Exzellenz, die wirtschaftliche Verwertung, die Lösung gesellschaftlicher Probleme), sie verfolgen je nach dem Grad der sozialen Einbettung unterschiedliche Interessen und verfügen über unterschiedliche Handlungsspielräume. Es gilt, Verbindungsmöglichkeiten an den Schnittflächen der drei Währungen zu finden, Potentiale zu identifizieren, herauszufinden, wo es Komplementaritäten der vorhandenen Bedürfnisse und Ressourcen gibt (wissenschaftliche Exzellenz ohne ökonomische Grundlage, wirtschaftliche Verwertung als reiner Selbstzweck und ohne Verbindung zu problemlösungsorientierten Fragestellungen, Missionsvorstellungen ohne die Ressourcen sie durchzuführen, oder ohne wissenschaftlich-rationale Basis, etc.).

Durch welche Arten von Akteuren aber werden nun die Verbindungen zwischen diesen Sphären hergestellt, sofern es welche gibt? Fügt man in die Karte der Bereiche und Förderinstrumente auch noch die Arten der Akteure ein, die in ihnen aktiv sind, und sieht sich die relativen Anteile an, ergibt sich das folgende Bild:

Abb. 29 - Verbindung der Bereiche und Förderinstrumente durch Kategorien von Akteuren 2009 - 2013 


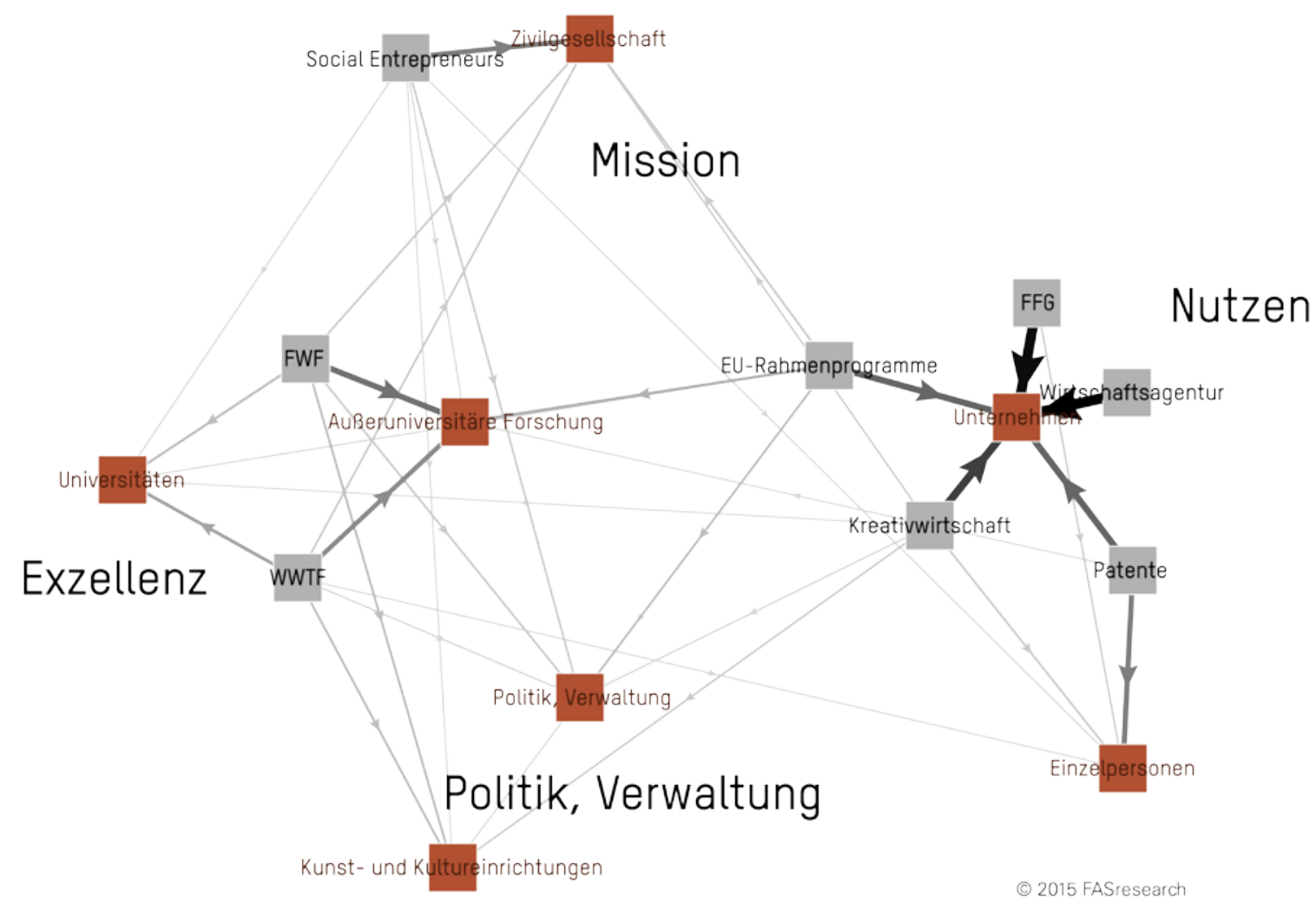

Datenquellen: FWF, WWTF, CORDIS, FFG, Wirtschaftsagentur, Depatisnet, Espacenet, FASresearchNetzwerkmonitor. Analyse: FASresearch

In der Darstellung oben repräsentieren die grauen Netzwerkknoten die Bereiche/Instrumente und die orangen die Arten von Akteuren. Die Pfeile stellen die relativen Anteile aus der Sicht der Bereiche/Instrumente dar, und zwar jeweils die fünf wichtigsten pro Akteurskategorie gemessen an den Prozentanteilen, die durch die Linienstärken repräsentiert sind. Es ist die oben angeführte Tabelle 26, die der Abbildung zugrunde liegt. Zum Beispiel machen Unternehmen 91,7\% der Akteure aus, die in Projekte der Wirtschaftsagentur involviert sind; ein entsprechend starker Pfeil verbindet Unternehmen und Wirtschaftsagentur. Die höchsten Prozentanteile verzeichnen die Unternehmen bei der Wirtschaftsagentur, der FFG, der Kreativwirtschaft, den Patenten und den EU-Projekten; entsprechend führen zu allen diesen Bereichen/Instrumenten Verbindungen.

In dieser Abbildung sind die missions- und problemlösungsorientierten Bereiche und Akteure nicht mehr an der Peripherie angesiedelt, sondern sie verfügen vielmehr über eine Brückenfunktion. Auf der linken Seite befindet sich eindeutig die exzellenzorientierte 
Sphäre (Universitäten, FWF, WWTF), und auf der rechten die Sphäre der Anwendungsorientierung und ökonomischen Verwertung (Unternehmen, FFG, Wirtschaftsagentur, Patente).

Wichtig ist nun, durch wen die Verbindung zwischen den Sphären der Exzellenz und der Verwertung hergestellt wird (in der Abb. 29 von unten nach oben betrachtet): Zunächst einmal durch die Politik und die Verwaltung, weil sie die rechtlichen und finanziellen Rahmenbedingungen setzt und aufgrund dieser Tatsache eine intermediäre Funktion ausübt. Weiters durch die außeruniversitäre Forschung, weil sie sowohl exzellenz- als auch aus anwendungsorientierte Institutionen beinhaltet. Und drittens durch die primär missions- und problemlösungsorientierte Zivilgesellschaft, die über die (auch) nutzenorientierte Kreativwirtschaft und die (auch) anwendungsorientierten EU-Projekte mit der Sphäre der Verwertung verbunden ist, und aber ebenso (wenn auch in geringerem Ausmaß) an Projekten teilnimmt, die in der Sphäre der Wissenschaft und Exzellenz angesiedelt sind.

Dass die missionsgetriebene Sphäre zwischen Exzellenz und Nutzen übersetzen könnte, weil sie in beiden Bereichen "sinnstiftende" Arbeit zu leisten vermag, zeigt sich auch daran, dass in ihr die Akteursdiversität hoch ist. Die Kreativwirtschaft befindet sich im folgenden Diagramm an erster Stelle, die Social Entrepreneurs an dritter Stelle:

\section{Abb. 30 - Akteursdlversität der Innovatlonsberelche und FörderInstrumente}

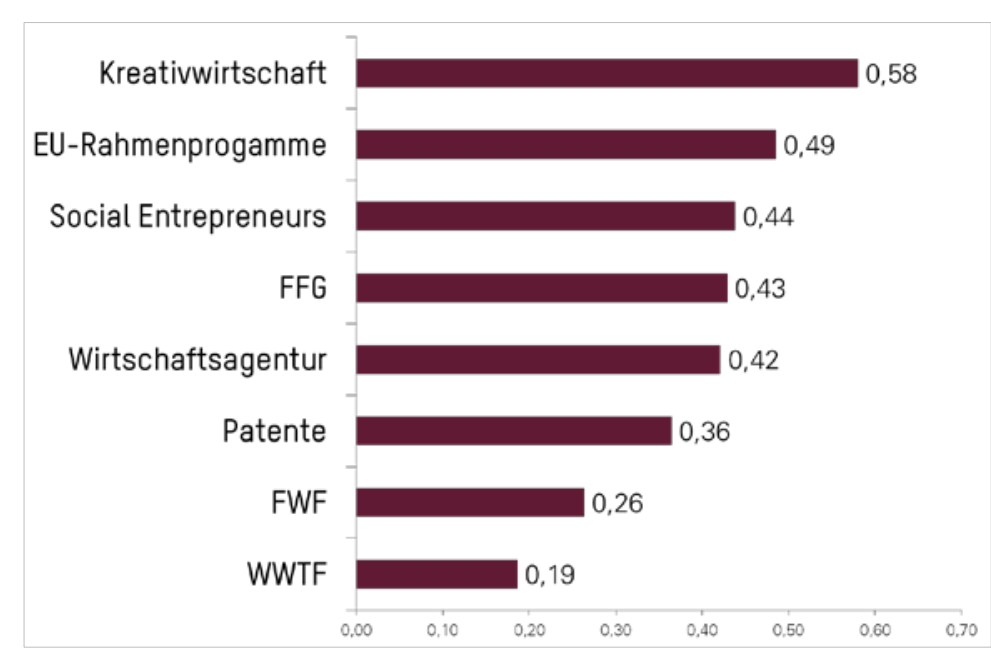

Datenquellen: FWF, WWTF, CORDIS, FFG, Wirtschaftsagentur, Depatisnet, Espacenet, FASresearchNetzwerkmonitor. Analyse: FASresearch 
Es zeigt, welche Bereiche sehr heterogen sind im Hinblick auf die Arten von Akteuren, die in ihnen vorkommen. ${ }^{37}$ WWTF und FWF adressieren in erster Linie Universitäten und auch die außeruniversitäre Forschung, darum weisen sie die geringste Akteursdiversität auf. Bei den Patentierungen dominieren die Unternehmen. Bei der Wirtschaftsagentur und der FFG wird das Feld schon breiter, die EU-Projekte adressieren die Forschung ebenso wie die Wirtschaft, und als am heterogensten im Sinne der Verteilung der Akteursarten erweist sich die Kreativwirtschaft, die Unternehmen unterschiedlichster Branchen, Interessenverbände, intermediäre Organisationen und Netzwerke (z.B. Coworking Spaces), Kunstund Kulturschaffende sowie Forscherinnen und Forscher, die sich mir der Kreativwirtschaft befassen, versammelt.

Welche konkreten Institutionen sind es nun, die als Übersetzer fungieren und die die drei Sphären der Innovation miteinander verbinden?

\subsection{Die Verbinder von Exzellenz und Nutzen}

Wir haben gesehen, dass es so gut wie keine Unternehmen gibt, die an FWF- oder WWTF-finanzierten Projekten teilnehmen, und wenn, dann handelt es sich um solche, die im Bereich der Wissenschaftskommunikation tätig sind, und nicht genuin in der Forschung. Jene Institutionen, die unter ihrem Dach Akteure versammeln, die sowohl in der Grundlagenforschung aktiv sind als auch Patente anmelden, sind entweder Universitäten oder gehören der außeruniversitären Forschung an. Allen voran gestellt müssen hier die Technische Universität Wien und die Universität für Bodenkultur werden, von denen Institute bzw. Personen in ALLEN Innovationsbereichen vorkommen, die in dieser Studie untersucht wurden, einschließlich der Kreativwirtschaft und den Social Entrepreneurs. Wei-

\footnotetext{
${ }^{37}$ Die Zahl ergibt sich aus 1 - dem Gini-Koeffizienten der Verteilung der Akteursarten (Außeruniversitäre Forschung, Kunst- und Kultureinrichtungen, Personen, Politik, Verwaltung, Universitäten, Unternehmen und Zivilgesellschaft) innerhalb jedes Bereichs. Je größer die Zahl, desto gleicher die Verteilung.
} 
ters ist das Austrian Institute of Technology (AIT) ein äußerst wichtiger Übersetzer in der Wiener Innovationslandschaft; mit Ausnahme der Social Entrepreneurs kommt es ebenso in allen drei Sphären der Innovation vor, und außerdem verbindet es auch innerhalb derselben unterschiedliche Bereiche (Grundlagen- und angewandte Forschung in der Wissenschaft, Life Sciences und IKT bei den Patenten). Auch die Universität Wien ist natürlich breit aufgestellt und kommt (mit Ausnahme der Wirtschaftsagentur im von uns untersuchten Zeitraum) in allen Innovationsbereichen vor.

Hier könnte man einwenden, dass diese Akteure aus einer Vielzahl an verschiedenen Untereinheiten (z.B. Instituten) bestehen, die in Wirklichkeit nichts miteinander zu tun haben. Wir sind trotzdem der Ansicht, dass es Sinn macht, von einheitlichen Akteuren zu sprechen, wenn es beispielsweise um das AIT oder um Universitäten geht, denn sie verfügen über Leitungsgremien, die sich auf die gesamte Organisation beziehen und sie nach auBen und innen vertreten (Geschäftsführung, Aufsichtsrat, Rektorat, Unirat, Senat usw.). Darüber hinaus kann ihr Name als eine Art Dachmarke aufgefasst werden, die den Zugang zu Ressourcen in Wissenschaft und Forschung erleichtern.

Weitere Akteure, die Exzellenz (FWF/WWTF) und Nutzen (Patente) verbinden, sind die Medizinische Universität Wien, das VRVis Zentrum für Virtual Reality und Visualisierung, die Veterinärmedizinische Universität Wien, die Akademie der Wissenschaften, das Institut für Molekulare Pathologie (IMP) und das Gregor Mendel Institut (GMI).

\subsection{Die Verbinder von Nutzen und Mission}

Bei den Akteuren, die sich in der Schnittmenge der Sphären der wirtschaftlichen Verwertung und der Mission bzw. Problemlösung befinden, handelt es sich hauptsächlich um kleinere oder mittlere Unternehmen. Ausnahmen bilden gemäß unserer Daten die Kapsch Trafficcom sowie die A1 Telekom Austria, die sowohl Patente aufweisen als auch im Zusammenhang mit der Kreativwirtschaft genannt werden. 
Die "kleineren" Verbinder von Nutzen und Mission sind zunächst einmal dort angesiedelt, wo sich die Bereiche der Medizin und der IKT überschneiden, also z.B. bei der Entwicklung gesundheitsbezogener Apps (mySugr GmbH) oder Internettechnologien (Diagnosia Internetservices). Auch das Rote Kreuz aus dem Bereich der Zivilgesellschaft ist als wichtiger Verbinder zu nennen, (abgesehen von seinen Hauptaufgaben) betreibt es Forschung (verfügt über ein eigenes wissenschaftliches Institut und ist an EU-Projekten beteiligt), ist unternehmerisch tätig und beteiligt sich an Projekten im kreativwirtschaftlichen Bereich bzw. zur Lösung gesellschaftlicher Probleme (z.B. Migration und Gesundheit).

Darüber hinaus werden Nutzen und Mission generell im Bereich von Internettechnologien, Multimedia, Software und App-Entwicklung verbunden (Zoomsquare im Bereich von Immobiliensuchmaschinen, die Semantic Web Company in Bezug auf Datentechnologien, Big Data, Informationsmanagement, die Datenwerk Innovationsagentur im Zusammenhang mit Online-Projekten und Social Media-Aktivitäten), die ovos GmbH (Design von Webseiten, Spielen und Apps unter anderem auch im Bildungsbereich) oder whatchado in Bereichen Aus- und Weiterbildung sowie Berufsfindung.

Eine weitere Falte von Nutzen und Mission bzw. Problemlösung bilden Medien und das Verlagswesen in spezialisierten Themenfeldern, wie etwa die Monopol Medien GmbH (The Gap in Bezug auf Popkultur, Biorama mit dem Themenbereich alternative und nachhaltige Lebensstile).

Und eine besonders wichtige Querschnittsmaterie gerade im Zusammenhang mit dem Konzept Industrie 4.0, das die "klassische" Industrie, die Digitalisierung sowie die Individualisierung der Produkte miteinander verknüpft, stellt das Design dar, in dem wir viele Akteure finden, die sowohl in den nutzen- als auch in den missionsorientierten Innovationsbereichen angesiedelt sind. EOOS Design stellt einen zentralen Player im DesignCluster des Kreativwirtschaftsnetzwerks dar, kann mehrere Patente vorweisen und hat auch an Projekten der EU-Rahmenprogramme teilgenommen. Spirit Design gehört ebenso zum Design-Cluster und ist bei FFG- und Wirtschaftsagentur-Projekten zu finden. Oder 
realitylab, dessen Selbstbeschreibung "Gestaltung von sozialen Prozessen" die Verknüpfung von Design (Medienfassaden, Architektur, Social Media) und Konzepten zur Bearbeitung bzw. Lösung sozialer Probleme (kollektive Prozesse bei gemeinnützigen Wohnprojekten) schon in sich birgt.

\subsection{Die Verbinder von Exzellenz und Mission}

Bei den Akteuren, die die Schnittmenge von Exzellenz und Mission bilden, geht es um jene, die an FWF- bzw. WWTF-Projekten beteiligt und die in den Netzwerken der Kreativwirtschaft bzw. der Social Entrepreneurs zu finden sind.

Das sind zunächst einmal Personen und/oder Institute an den Universitäten, die sich mit gesellschaftlichen Problemlagen im weitesten Sinne beschäftigen. An der Universität für Bodenkultur etwa im Zusammenhang mit Ökologie, Klimaschutz oder alternative Energien; an der Technischen Universität in Bezug auf innovative Verkehrstechnologien, erneuerbare Energien oder auch im Hinblick auf Wissenschaftskommunikation, Bildung und Jugend.

Beim AIT finden wir Personen, die sich mit Innovationsmanagement bzw. den gesellschaftlichen Voraussetzungen für die Entstehungen von Innovationen auch in einem allgemeineren Sinn bzw. von sozialen Innovationen befassen.

An der Universität Wien gibt es eine Reihe von problemlösungsorientiert tätigen Menschen, wie etwa am Institut für Psychologie oder in den Bildungswissenschaften (Arbeit und Soziales, Migration und Bildungskonzepte) sowie an der Fakultät für Informatik, wo man sich unter anderem mit der Frage nach der Wissenschaftskommunikation gerade im Zusammenhang mit den Natur- und den formalen Wissenschaften (Mathematik, Computerwissenschaften) auseinandersetzt, die auch in den von uns identifizierten Netzwerken (in der Grundlagenforschung, bei den Publikationen im technischen Bereich und bei den 
Patenten) eine so wichtige Rolle als Übersetzer spielen. Auch die Kinderuni und alle, die daran beteiligt sind, spielen hier eine wichtige Rolle.

In den Netzwerken der Social Entrepreneurs sind auch Personen der Wirtschaftsuniversität Wien von zentraler Bedeutung, die zum Thema Nonprofit Management oder Entrepreneurship und Innovation arbeiten.

Die Kunstuniversitäten sind ebenfalls wichtige Übersetzer zwischen Wissenschaft und Mission. Das betrifft zunächst einmal das Thema Design (Universität für angewandte Kunst, Akademie der bildenden Künste), weiters das Verhältnis von Kunst und Gesellschaft (neben den bereits genannten die Universität für Musik und darstellende Kunst z.B. im Hinblick auf die Geschichte und Bedeutung der Popmusik) sowie die Architektur (Architekturzentrum Wien). Das Technische Museum schließlich ist ebenfalls Teil des Netzwerks von Kultureinrichtungen, die eine wesentliche Rolle für die Wissenschaftskommunikation spielen.

Und schließlich müssen die zahlreichen Wissenschaftseinrichtungen hervorgehoben werden, die in der Erforschung von sozialen Problemen aktiv und zum Teil auch zivilgesellschaftlich engagiert sind (WIFO und IHS bei Wirtschaft und Arbeit, die FORBA im Zusammenhang mit Beschäftigung, Arbeitslosigkeit und Arbeitsbedingungen; die Statistik Austria bei der Erforschung von Einkommen, Ungleichheit und Armut).

Die in den letzten drei Abschnitten genannten Akteure sind nicht willkürlich ausgewählt, sondern es handelt sich um Personen und Institutionen, die in den Innovationsbereichen, Förderinstrumenten und Netzwerken, auf die sich unsere Studie bezieht, prominent vertreten sind. Es gilt unserer Ansicht nach, die Querverbindungen, die Integration auf horizontaler Ebene zwischen diesen Akteuren zu stärken, und zwar im Bewusstsein dessen, dass für diese Akteure unterschiedliche Währungen von Bedeutung sind, dass sie entsprechend ihrer Positionen in den sozialen Feldern unterschiedliche Haltungen aufweisen, dass sie unterschiedlich groß und unterschiedlich schnell sind - und dass es aber genau diese Unterschiede sind, die ihre Zusammenarbeit als sinnvoll erscheinen lassen, weil zwi- 
schen ihnen starke Komplementaritäten hinsichtlich der Ressourcen, über die sie verfügen, bestehen.

Ans Ende dieses Abschnitts stellen wir eine Netzwerkkarte, in die alle Akteure eingetragen sind, die im Zeitraum 2009 bis 2013 in mindestens zwei Innovationsbereichen, die ebenfalls als Netzwerkknoten dargestellt sind, aufscheinen. Die Position im Netzwerk zeigt an, in welchem Sektor der Innovation (Exzellenz, Nutzen, Innovation) sich die Institutionen tendenziell befinden. An der Peripherie des Netzwerks sind jene Akteure angesiedelt, die eher auf eine bestimmte Sphäre spezialisiert sind. Je mehr man sich dem Zentrum zuwendet, desto eher trifft man auf Player, die unterschiedliche Sphären miteinander verbinden. 


\section{Abb. 31 - Wiener Institutionen und Ihre Betelligung an Förderinstrumenten und Innovationsbereichen 2009 - 2013}

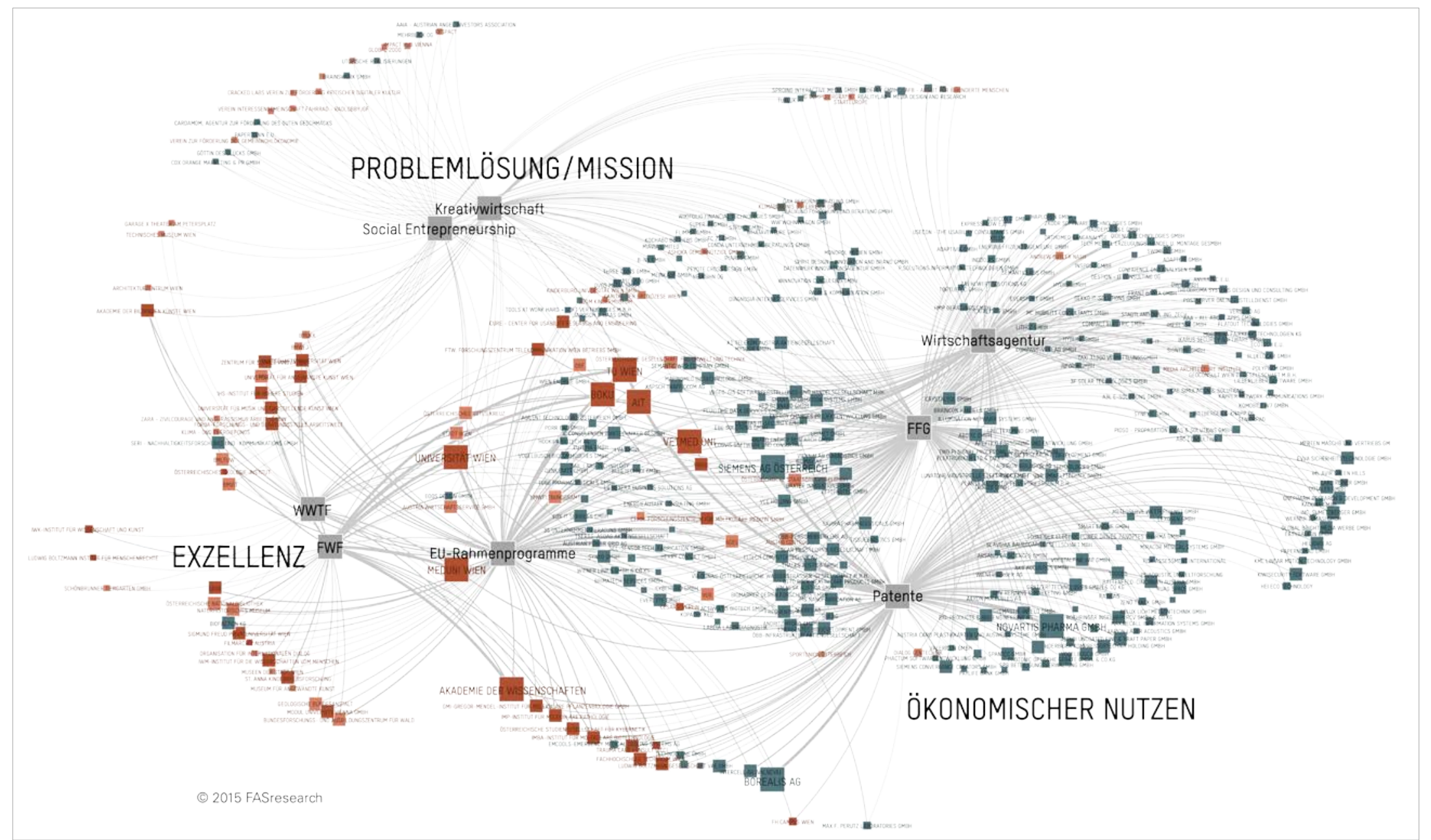

Datenquellen: FWF, WWTF, CORDIS, FFG, Wirtschaftsagentur, Depatisnet, Espacenet, FASresearch-Netzwerkmonitor. Analyse und Grafik: FASresearch. Graue Netzwerkknoten: Förderinstrumente bzw. Innovationsbereiche. Blau: Unternehmen. Dunkelbraun: Wissenschaft und Forschung. Hellbraun: Sonstige Institutionen. Linien zeigen an, dass ein 


\section{FAS research}

Akteur in einem bestimmten Bereich oder Förderinstrument vorkommt. Abgebildet sind Akteure, die im angegebenen Zeitraum in mindestens zwei Bereichen vertreten waren ( $\mathrm{N}=409$ von 2.867 Institutionen). 
Zusammenfassung der Gesamtschau:

- Die Verschneidung aller acht Datenquellen zeigt die drei durch die Währungen der Innovation voneinander getrennten Cluster: den exzellenzgetriebenen Cluster FWF/WWTF, den durch anwendungsorientierte Forschung und Nutzenorientierung charakterisierten Cluster EU-Rahmenprogramme/FFG/Wiener Wirtschaftsagentur/Patente sowie einen missions- und problemlösungsorientierten Bereich (Kreativwirtschaft, Social Entrepreneurs).

- Die EU-Rahmenprogramme stellen die Brücke zwischen Exzellenz und Nutzen her, und die Kreativwirtschaft jene zwischen Nutzen und Mission, wobei hier die Wirtschaftsagentur Wien und die FFG eine besondere Rolle spielen.

- FFG und Wirtschaftsagentur weisen dem statistischen Erwartungswert entsprechende Schnittmengen mit der Kreativwirtschaft auf, im Hinblick auf die Beziehungen zu den Social Entrepreneurs jedoch sind die Überschneidungen deutlich geringer als statistisch erwartet. Das bedeutet, dass es im Gegensatz zur Bearbeitung der Zielgruppe Kreativwirtschaft bei der Zielgruppe der Social Entrepreneurs noch Potential gibt, was den Aufbau und die Stärkung der Beziehungen betrifft.

- Zwischen Nutzen und Mission sind es fast ausschließlich kleine Analyse- und Designunternehmen (d.h. der Kreativwirtschaft zuzurechnende KMUs), die die Brückenarbeit leisten.

- Was die Sphären der Exzellenzforschung und der Wirtschaft angeht, sind es allem die außeruniversitären Forschungseinrichtungen, die die Verbindung herstellen.

- Zwischen den Sphären der Exzellenz und der Mission werden die Brücken durch die Universitäten, durch Forschung (AIT, FORBA) sowie durch Kultureinrichtungen (Museen) hergestellt.

- Insgesamt zahlreiche "White Spaces" entlang der unterschiedlichen WährungsDimensionen, wobei die Kluft von der universitären Exzellenz zum Feld des Nutzens 
und zur Mission deutlich höher ausgeprägt ist als etwa die zwischen Nutzen und Mission. 


\section{Empfehlungen}

Die Smart City der Zukunft ist jene, in der die drei Innovationsfelder der Wissenschaft (Exzellenz, Status), der Wirtschaft (Nutzen, Privateigentum) und der Zivilgesellschaft/Politik (Mission, Commons) aufeinander Bezug nehmen und immer wieder "ein Stück des Weges gemeinsam gehen". Insbesondere Business-Model-Innovationen entstehen in Übergangszonen zwischen hierarchischen, egalitären und individualistischen Innovationskulturen und sind oft das Produkt von hybriden Modellen ("Heterarchien"). Die hier vorliegende Studie brachte durch die Verschneidung von neun unterschiedlichen Datenquellen zum Vorschein, dass innerhalb der einzelnen Felder (Wissenschaft, Wirtschaft, Zivilgesellschaft), sowie zwischen diesen Bereichen interessante Bruchlinien (sogenannte „White Spaces") ausgemacht werden können, deren Bearbeitung die Innovationskultur des Standorts Wien wesentlich stärken könnte.

Die folgenden Empfehlungen sind vom Geiste der theoretisch und empirisch begründbaren Überlegung getragen, dass die in der Studie nachgezeichneten Innovationsnetzwerke keine rein maschinellen "Devices" sind, sondern erst durch eine spezifische "Kultur" entstehen und sich nur über ein besonderes "Mindset" immer wieder erneuern können. Die Smart City benötigt ein tiefes Verständnis dafür, wie eine Ökologie der permanenten Erneuerung ausgeprägt sein muss, die sich eben vor allem dadurch auszeichnet, dass sie die Grenzen zwischen Exzellenz, Nutzen und Mission immer wieder aufhebt und daraus transformative, radikale Lösungen hervorgehen, sowohl was Produkte als auch was Prozesse und Geschäftsmodelle anbelangt. Das radikal Neue liegt immer im „Dazwischen“, und dieses kann nur erkundet werden, wenn die Akteure aus ihren „Silos” und Komfortzonen herausgehen, ganz im Sinne einer Kultur der "Explorer" und "Pioniere" - was eher dem angelsächsischen Typus entspricht - aber auch ganz in der Tradition einer Kultur der „Begegnungen“, was eher der Stadt Wien in ihrer historischen Mission entspricht. 
- Es wird empfohlen, das Narrativ der Smart City Vienna durch eine Neuinterpretation von „Wien als Ort der Begegnung” zu erweitern. Wien als Ort der Begegnung zwischen exzellenter Wissenschaft und Mission, zwischen Mission und Wirtschaft, zwischen Wirtschaft und Wissenschaft. Damit verbunden die Interpretation der Kultur der Smart City als eine der offenen, partizipativen, reflexiven Übersetzungen, Dialoge, CoKreationen, Co-Innovationen zwischen hierarchischen (Universitäten, große Leitbetriebe), egalitaristischen (NGOs, Communities der Kreativwirtschaft) und individualistischen (Start-ups) Domänen der Innovation.

- Mit dem Narrativ "Wien als Stadt der Begegnung" verbunden sollte auch überlegt werden, wie das Thema "Wien als Stadt der sozialen Innovationen" in eine Smart City-Philosophie passt. Fakt ist, dass das Thema der sozialen Mobilität im Sinne der vertikalen Durchlässigkeit unserer Gesellschaft ein wesentliches gesellschaftspolitisches Ziel sein muss, aber gleichermaßen das zur Zeit von niemandem aufgegriffene Problem der horizontalen Durchlässigkeit eine große Herausforderung darstellt: Wie können Universitätsangehörige in Unternehmen, in zivilgesellschaftliche Einrichtungen und wieder zurück umsteigen, wie kommen wir zu einer "brain circulation", indem Menschen verschiedene Rollen im Laufe ihrer Karrieren durchlaufen können und nicht in einer stecken bleiben: ForscherInnen, ManagerInnen, GründerInnen, WeltretterInnen, InvestorInnen. In dieser Rollendurchlässigkeit liegt nebenbei bemerkt einer der wesentlichen "Geheimnisse" des Silicon Valley begründet; die Verbindungen zwischen etablierten Universtäten und Unternehmen, Design-Labs, Start-ups, Foundations und VCs werden dadurch gewährleistet, dass dieselbe Person im Laufe ihres Lebens diese verschiedenen Rollen einnehmen kann.

- Ein systematischer Stakeholder-Prozess, der exakt die Frage der Schnittstellen zwischen Exzellenz, Nutzen und Mission vor dem Hintergrund der Herausforderungen und Zielsetzungen der Smart City zum Gegenstand hat, wäre angeraten. Da durch die vor- 
liegende Studie die Key-Player in den einzelnen Bereichen bekannt sind, wäre die Frage, wie etwa die Zusammenarbeit in Innovationsprozessen zwischen etablierter Industrie (Kapsch, Siemens, Borealis, Mondi etc.) und der Kreativwirtschaft („Hub Vienna”) verbessert werden könnte, mit ebendiesen Akteuren im Rahmen von moderierten Workshops, Round Tables, Kamingesprächen zu besprechen und durch Bereitstellen eines „Prototyping Funds" (siehe unten) daraus generierte Projekte zu fördern.

- Es wird die Einrichtung eines „Smart City Prototyping Funds" vorgeschlagen, der analog zum Innovationsscheck-Modell der FFG dazu dient, durch "small grants" (maximal 5.000 Euro) Experimente in der cross-sektoralen (Nutzen-Mission-Exzellenz) und in der Cross-Scale-Zusammenarbeit (Groß und Klein) zwischen Universitäten, Social Entrepreneuren und Kreativwirtschaft zu stimulieren. Der Smart City Prototyping Fund sollte insbesondere rund um Themenfelder zum Einsatz kommen, in denen es wesentliche Stärkefelder in Wien gibt: wie etwa rund um die Themen Licht, Wasser und Mathematik.

- Es wird empfohlen, die Etablierung eines „Smart City Open Innovation Labs” zu überprüfen. Das sollte ein Ort sein, an dem Personen aus großen Unternehmen oder Universitäten mit hierarchischer Kultur gemeinsam mit Akteuren aus egalitären Settings (Kreativwirtschaft) sowie Personen mit individualistischer Kultur (klassische Entrepreneure) an Lösungen innerhalb der durch die Smart City-Vision vorgegebenen Bereiche (Smart Production, Governance, Energie und Mobilität, Demographie, Leben etc.) arbeiten. Dieses Lab sollte als eine Art forschender Think-Tank der neuen Art (DesignLab) ein Prototyp für eine hybride Organisation sein und im Sinne des Konzepts der problemorientierten Forschung transdisziplinäre, missionsorientierte CoKreationsprozesse stimulieren. 
- Generell könnten im Sinne der oben beschriebenen horizontalen Durchlässigkeit als möglichem Inhalt eines „Wiens der sozialen Innovationen" neue Sabbatical- und Bildungskarenz-Lösungen für Wiener Arbeitskräfte im Innovationsbereich (egal ob Wirtschaft, Universitäten oder Verwaltung) überlegt werden. Etwa könnte ForscherInnen aus großen Leitbetrieben, aber auch MitarbeiterInnen aus der Verwaltung für eine Zeit in Co-working Spaces oder bei Key-Playern der Kreativwirtschaft (Hub, EOOS etc.) eine "Auszeit" aus hierarchischen Settings ermöglicht werden. Corporate Volunteering oder Sabbaticals in forschenden kulturellen oder zivilgesellschaftlichen Einrichtungen (z.B. Jüdisches Museum, Greenpeace, Rotes Kreuz etc.) könnten zu einer stärkeren Verbindung im Dreieck Mission-Exzellenz-Nutzen führen.

- Experimente mit dualen Universitätslehrgängen an etablierten Universitäten, aber auch an den Wiener FHs nach dem Modell der Lehrlingsausbildung könnten auch interessante Anstöße liefern für die Überwindung der (kulturellen) Grenzen zwischen Wissenschaft, Wirtschaft und Zivilgesellschaft.

- Generell gilt es Überlegungen anzustellen, wie ungenutzte Patente („Orphaned technologies") eingesetzt und demobilisierte, "frustrierte", fatalistisch gewordene ForscherInnen aus Universitäten oder großen Unternehmen („Disenfranchised Scientists") verstärkt in Start-up Aktivitäten oder im Sinne der horizontalen Durchlässigkeit in anderen Feldern (NGOs, öffentliche Verwaltung etc.) eingebunden werden können. Im Silicon Valley entstehen Start-ups nicht ausschließlich durch „junge Wilde”, sondern sehr oft auch über Ausgründungen von älteren, erfahrenen IngenieurInnen und ManagerInnen. Die Patentanalyse zeigt eindeutig, dass in Wien kaum aus etablierten Unternehmungen heraus technologie- bzw. patentbasierte Start-ups entstehen. Unter dem Titel „Unlock Potential" müsste man an Wegen arbeiten, wie wir innerbetriebliche ForscherInnen /InnovatorInnen auf dem Abstellgleis aus größeren Organisationen "herausholen“ und in ein neues, gründerfreundliches „Biotop” versetzen könnten. Vielleicht 
könnte man hier Private-Public-Partnerships-Finanzierungsprogramme für "Senior Researcher Startups" anbieten.

- Generell wäre eine spezielle Untersuchung und Identifikation über „Orphaned Technologies“ (via Patentanalyse und ExpertInneninterviews) anzustellen; nach Schätzungen in den USA werden im Schnitt 80-90\% aller Entdeckungen innerhalb großer Organisationen aus Gründen der Firmenpolitik, wegen Konkurrenzstrategien oder aufgrund fehlenden Nutzens „ins Abseits” gestellt (Stadler 2008). Auch hier gilt, dass das Rad nicht immer neu erfunden werden muss. „Wenn Wien wüsste, was Wien weiß!” In der Smart City der Zukunft geht es nicht immer nur um Neu-Entdeckungen, sondern auch um Wieder-Entdeckungen und die Freisetzung nicht realisierten Potentials.

- Ähnlich brachliegendes Potential gibt es bei den nicht-akademischen „Bastlern” und „Tüftlern", die, wie die Patentanalyse gezeigt hat, viele hunderte Einzelpersonen in Wien ausmachen. Sogenannte "Maker meets Industry - Days könnten hier Annäherungen bilden, die künftige Bedeutung des „Maker Movements" in einer dezentral produzierenden Industrie 4.0-Umwelt sollte nicht unterschätzt werden, vor allem für die Erarbeitung und Implementierung von "Place based solutions".

- Die Smart City Vienna benötigt ein der Komplexität der Herausforderungen angemessenes Governance-Modell mit neuen Möglichkeiten der Partizipation und der Entscheidungsfindung. Es wird empfohlen hier einen eigenen Schwerpunkt an der Schnittstelle von Wirtschaftsuniversität, Technischer Universität, außeruniversitärer Forschung (z.B. AIT, IIASA) und der kreativen Designszene (EOOS, Hub Vienna) zu etablieren. Design-Thinking ist eine Form der Entscheidungsfindung unter schwierigen Bedingungen („Robust Decision Making“, „Wicked Problem Management“). Künftige ManagerInnen auf der WU könnten sehr viel von DesignerInnen und ihren prozessorientierten Problemlösungsstrategien lernen. Wien könnte hier zu einer Vorreiterin für 
Governance und Leadership unterstützende Technologien werden. Die in der Studie herausgearbeiteten auffälligen strukturellen Lücken zwischen IIASA und AIT, sowie zwischen TU und WU - die, wie die Netzwerkanalyse zeigt, alle an Computational Management und Decision-Making Technologies arbeiten - wären hier ein relevantes Betätigungsfeld.

- Die Smart City der Zukunft ist eine Stadt neuer, innovativer, partizipativer GovernanceTools, aber auch neuer, fortgeschrittener Coaching-, Moderations-, Konferenz- und Therapietechniken. Das Wissen der PsychologInnen, TherapeutInnen, TrainerInnen etc. ist ein unabdingbares Wissen in der Arbeit an den Begegnungen zwischen Nutzen, Exzellenz und Mission und ihren unterschiedlichen Codes. Bei psychologisch und therapeutisch geschulten Moderations- und ProzessspezialistInnen ist Exzellenz erfolgskritisch. Es wird daher empfohlen, einen eigenen Forschungs- und Anwendungsschwerpunkt für Coaching, Therapie und Moderation im Zusammenhang mit Zielen und Themen der Smart City zu etablieren, einen sogenannten Smart City Facilitation Hub. Wien könnte einen neuen Forschungs- und Innovationsschwerpunkt für Moderationsund Prozessbegleitung, Coaching etc. aufbauen und so seinem Ruf als Stadt der Psychoanalyse/Therapie und als Ort der Begegnung im doppelten Sinne (mit dem Ich und dem Anderen) wieder gerecht werden.

- In eine ähnliche Kerbe schlägt die Empfehlung, Wien als Zentrum von „Deep Data”Analysen zu machen. Wo, wenn nicht in Wien als Ort der "Tiefe" (Psychoanalyse), müsste jenes Thema bearbeitet werden, dass das "next big thing" werden könnte, nämlich der Weg von den „big data” zu den „deep data”. Daten - und seien sie auch noch so umfangreich - haben ohne Kontexte, ohne Verstehen keine Tiefe, sie sind ohne Bedeutung. Wäre das nicht ein Thema, das auch eine Brücke zwischen der Informatik und den Geisteswissenschaften herstellen könnte? Wären es hier nicht die Geistesund SozialwissenschafterInnen, die mit ihren "langsameren" Untersuchungsobjekten 
und Skalen dazu beitragen könnten, aus den schnellen Variablen einen Sinn zu destillieren (big data sind immer auch schnelle Daten, d.h. sie verändern sich rasch und sind daher aber auch rasch obsolet)? Es wird empfohlen, das Thema "deep data" als Brückenthema gemeinsam mit den Stakeholdern zu explorieren.

- Das notorische, strukturelle Problem des im internationalen Vergleich zu geringen Investitions- und Risikokapitals für privat finanzierte Forschung und Start-ups könnte durch die Etablierung und Stärkung einer gemeinnützigen Wiener Stiftungskultur aktiv angegangen werden. Der gemeinnützige Stiftungssektor würde auch eine ideale Netzwerkbrücke zwischen privaten, staatlichen und zivilgesellschaftlichen Akteuren spielen. Es wird empfohlen, gemeinsam mit zentralen Akteuren der Finanzwirtschaft (Erste Bank, Bank Austria, VIC), mit Akteuren des gemeinnützigen Stiftungswesens, dem WWTF, dem Wissenschaftsministerium sowie den Universitäten Wiens im Rahmen einer gemeinsamen Tagung die Möglichkeiten der Zusammenarbeit auszuloten. Angelpunkt dabei ist die Frage, welche eigenständigen Felder, die ähnlich der deutschen Stiftungslandschaft eben nur von Stiftungen getragen werden können, identifiziert werden können. Stiftungen wollen nicht nur der Notnagel für ausfallende Ausgaben der öffentlichen Hand sein, sondern zusätzliche, eigenständige Projekte entwickeln, was ja wiederum die Lösungsdiversität (vgl. Ashby's "Law of requisite variety") der Stadt erhöhen würde. Das Thema der Umsetzung der Vision von wahrer problemorientierter Forschung sollte hier ganz oben auf der Agenda stehen. Zur Zeit ist transdisziplinäres Forschen auf den Universitäten auf Grund der Spielregeln der Exzellenz ein "Karrierekiller", stiftungsfinanzierte Forschungsprojekte etwa im Rahmen des oben beschriebenen Smart City Labs, des WWTF oder anderer Einrichtungen könnten hier einen neuen und eigenständigen Spielraum eröffnen.

\section{Weitere Maßnahmen:}


- Generell wird die Fortsetzung und Vertiefung des aus netzwerkanalytischer Sicht äußerst erfolgreichen und für die Überbrückung von „White Spaces” paradigmatischen WWTF-Ansatzes „Mathematik und..." dringend empfohlen. Überprüft werden sollten Möglichkeiten, dieses Modell auch auf die Domänen des Nutzens und der Mission auszuweiten, Calls etwa mit den Titeln „Design und...", "Wasser und...", „Licht und...", „Bildungswissenschaften und...", „Komplexitätsforschung und...” könnten wesentliche strukturelle Löcher innerhalb der Wiener Forschungslandschaft schließen helfen.

- Das vereinigende Thema einer Industrie 4.0 getriebenen Smart City ist das Thema IKT. Die Patentlandkarte Wiens zeigt, dass es zwischen den beiden Stärkefeldern IKT und Life-Sciences auf der Unternehmensseite zur Zeit noch zu wenige Beziehungen gibt, Brückenthemen der Health Technologies, Bioinformatik und Computational Medicine bilden hier Ansatzpunkte. Die wesentliche Brücke sollte allerdings das von allen Stakeholdern gemeinsam getragene Anliegen sein, die Coding Literacy (also das Erlernen und Anwenden von Programmiersprachen) zu erhöhen und Coding neben Deutsch und Englisch als die für die Zukunft wesentliche zweite/dritte lebende Fremdsprache zu etablieren. Es könnte Bemühungen geben, für einen Viennese Day of Coding oder im Rahmen einer wöchentlichen Hour of Coding die Kompetenz des Codens in ALLE soziodemografischen Milieus Wiens zu transportieren, insbesondere aber ForscherInnen der Geistes- und Sozialwissenschaften dafür zu gewinnen.

- Es wird empfohlen zu überprüfen, wie eine verstärkte Förderung von Computational Social Sciences die Sozial- und Geisteswissenschaften, die sich mit Ausnahme der Geschichtswissenschaften und der Archäologie zur Zeit in Wien sowohl in der Welt des FWF als auch im EU-Kontext am absteigenden Ast befinden, wieder "zurück ins Spiel” zu bringen. Angesichts der „Flachheit” der „big data“-Welt für die kulturelle und intellektuelle künftige Entwicklung der Stadt eine sicherlich lohnende Anstrengung. 
- So wie die Mathematik, Informatik, Geowissenschaften oder Psychologie Brückenwissenschaften zwischen Science und Humanities sind, so sind alle mit Bild und Visualisierung befassten Disziplinen (Infographic Design) wichtige Übersetzungsfächer zwischen der Welt der Wissenschaft („Exzellenz”), des Nutzens und der Mission. Es wird empfohlen, neben der stärkeren Nutzung der Computational Sciences als Verbinder zwischen den Welten die Möglichkeiten zu prüfen, wie vor dem Hintergrund „Infografiken" und "Gamification" die Trennung zwischen der TU, den Kunstuniversitäten, der Graphic Design Community sowie der Community der Spieleentwickler verringern könnten.

\section{Limitations und mögliche nächste Schritte}

Es gibt zumindest drei Datenquellen, die in der vorliegenden Studie fehlen, und deren Inkludierung das Gesamtbild abrunden könnte: die Förderdaten des Austria Wirtschaftsservice (AWS), des INiTS, sowie der von der FFG geförderten Akteure, die keine Unternehmen sind. 


\section{Key-Deliverables der Studie}

- Webbasiertes Tool zur Explorierung der Wiener Patentlandschaft inklusive der Zitationsbeziehungen zwischen den Technologieklassen und den zugehörigen Anmeldern.

- Netzwerkkarten der Wiener Grundlagenforschung, der Partizipation an EU-Projekten, der Publikationen im Life Science- und im Technologie-Bereich sowie der Zitationsbeziehungen zwischen Wiener und internationalen Patent-Anmeldern.

- Analyse und Auswertungen in Form von mehr als 250 PPT-Folien.

- Präsentation der Zwischenergebnisse vor ausgewählten Schlüsselspielern der Wiener Innovationslandschaft im Rahmen eines Sounding Boards.

- Umfassender Endbericht mit Politikempfehlungen.

\section{Abbildungs- und Tabellenverzeichnis}

Abb. 1 - Die drei Währungen der Innovation

Abb. 2 - Auszug aus der Espacenet-Patentdatenbank mit einem Patent von Siemens

Österreich

Abb. 3 - Patentzitationen: Zitationsrichtung und Wissenstransfer

Abb. 4 - Wikipedia-Artikel mit „Vienna” nach der Anzahl der Zugriffe 2014.

Abb. 5 - „Vienna"-Wikipedia-Artikel nach der durchschnittlichen Zunahme an monatlichen Zugriffen 2014 30

Abb. 6 - Anzahl der FWF-Projekte, Wien und österreichweit im Zeitraum 1995 - 2013.....31 Abb. 7 - Netzwerk der Wissenschaftsdisziplinen der Wiener FWF-Projekte 1995 - Oktober 2014 . .35

Abb. 8 - Prozentanteil der WWTF-Programme an den Gesamtaufwendungen 2009 - 2013

Abb. 9 - Beteiligung von Institutionen an WWTF-Förderprogrammen 2009 - 2013

(Ausschnitt) 
Abb. 10 - Anzahl der Pubmed-Publikationen mit Wiener Beteiligung 2009 - 2013 .45

Abb. 11 - Netzwerk der Wiener Publikationen im Bereich der Life Sciences 2010 - 201447

Abb. 12 - Netzwerk der Wiener Publikationen im Bereich Technologie 2002 - 2013;

Schlagworte, die durch mindestens drei Publikationen verbunden sind 51

Abb. 13 - Anzahl der EU-Projekte mit Wiener Beteiligung 2009 - 2013.

Abb. 14 - Netzwerk der Kooperation in EU-Projekten 2002 - 2014 . .58

Abb. 15 - Anzahl der Patente 1990 - 2013, Wien und übrige Bundesländer.

Abb. 16 - Entwicklung der Wiener Patentzahlen nach Anmelderkategorien (ohne

Einzelpersonen) $2002-2013$

Abb. 17 - Patente von Wiener Anmeldern mit internationaler und nationaler Gültigkeit

$2002-2013$

Abb. 18 - Gini-Koeffizient der Verteilungen der Patentzahlen auf die Wiener Anmelder

$2009-2013$

Abb. 19 - Patent-Sektionen nach Größe und Momentum, Wiener Patente 2009 - 2013 ..71

Abb. 20 - Umwandlung des Patentnetzwerks in ein Patentklassennetzwerk.

Abb. 21 - Patent-Unterklassen nach dem Prozentanteil der Klassen, die sie zitieren,

Wiener Patente 2009 - 2013.

Abb. 22 - Zitationsnetzwerk der Patentklassen, Wiener Patente 1979 - 2014.

Abb. 23 - Die Cluster der Technologiefelder nach Momentum und Anzahl der Wiener

Patente 2009 - 2013

Abb. 24 - Netzwerk der Patentzitationen zwischen Wiener und internationalen Anmeldern $2004-2014$

Abb. 25 - Patentportfolio Wiens: IPC-Klassen nach der Anzahl der Wiener Patente 1979 2014 und nach dem Durchschnittsalter der Patente 98 Abb. 26 - Patentportfolio Wiens: IPC-Klassen nach der Anzahl der Wiener Patente 2009 2013 und nach dem Momentum 2009-2013. . .99

Abb. 27 - Verbindung der Innovationsbereiche durch Wiener Institutionen 2009 -2013 107 


\section{Abb. 28 - Vergleich der beobachteten und der erwarteten Schnittmengen zwischen den Bereichen -}

Abb. 29 - Verbindung der Bereiche und Förderinstrumente durch Kategorien von

Akteuren 2009 - 2013

Abb. 30 - Akteursdiversität der Innovationsbereiche und Förderinstrumente

Abb. 31 - Wiener Institutionen und ihre Beteiligung an Förderinstrumenten und

Innovationsbereichen 2009 - 2013

Tabelle 1 - Übersicht: Wertehaltungen, Einstellungen und kulturelle Rollen einer Innovationsökologie

Tabelle 2 - Aufbau der ÖFOS 2012 anhand des Beispiels Medizinische Biotechnologie (Code 3040)

Tabelle 3 - Aufbau der IPC Patentklassifikation.

Tabelle 4 - Wissenschaftsdisziplinen nach der Anzahl der Projekte mit Wiener Beteiligung $2009-2013$

Tabelle 5 - Institutionen (Organisationsebene) nach Anzahl der FWF-Projekte 2009 - 2013

Tabelle 6 - Institutionen (Institutsebene) nach Anzahl der FWF-Projekte 2009 - 2013 ........39

Tabelle 7 - Institutionen nach der Zahl der WWTF-Projekte 2009 bis 2013

Tabelle 8 - Wiener Life Science Publikationen 2010 - 2013: die wichtigsten SCOPUS-

Klassen.

Tabelle 9 - Die wichtigsten Felder Wiener Publikationen im Technologiebereich (IEE-

Datenbank, Schlagworte)

Tabelle 10 - Subjects der EU-Projekte mit Wiener Beteiligung 2009 - 2013 sowie der durchschnittlichen jährlichen Zunahme an Projekten („Momentum“).

Tabelle 11 - Die Schlüsselspieler der Teilnahme an EU-Projekten 2009-2013.

Tabelle 12 - SIC-Klassen der FFG nach der Anzahl der Projekte 2012 - 2014

Tabelle 13 - Technologiefelder nach der Zahl der Wiener Patente 2009 - 2013 
Tabelle 14 - Die zehn häufigsten Patentklassen mit den jeweils drei wichtigsten Wiener Anmeldern 2009 - 2013

Tabelle 15 - IPC-Klassen mit dem stärksten Momentum, Wiener Patente 2009 bis 2013.73

Tabelle 16 - Patentklassen mit dem größten Momentum 2009 - 2013 und den

zugehörigen Wiener Anmeldern

Tabelle 17 - Patentklassen mit fallenden Patentzahlen, Wiener Patente 2009 - 2013 ..........75

Tabelle 18 - Technologiefelder mit den Patenten Wiener Anmelder 1979 - 2014 mit dem geringsten Durchschnittsalter; nur Unterklassen mit mindestens fünf Patenten im angegebenen Zeitraum

Tabelle 19 - Unternehmen mit Sitz in Wien nach der Zahl der Patente 2009 -2013

Tabelle 20 - Forschungsinstitutionen mit Sitz in Wien nach der Zahl der Patente 2009 -

2013 (Depatisnet, Espacenet)

Tabelle 21 - Wiener Patentanmelder mit den meisten Zitationen pro Patent.

Tabelle 22 - Wen zitieren Wiener Patente - Backward Citation, Input an Know-how nach

Wien 2004 - 2014.

Tabelle 23 - Wer zitiert Wiener Patente - Forward Citation, Output an Know-how aus

Wien 2004 - 2014

Tabelle 24 - Die Akteure der untersuchten Innovationsbereiche 2009 - 2013 nach

Kategorien 105

Tabelle 25 - Verteilung der Akteure auf die Bereiche (absolute Zahlen,

Mehrfachnennungen möglich) 106

Tabelle 26 - Verteilung der Akteure auf die Bereiche (Spaltenprozent, Mehrfachnennungen möglich) 106 


\section{Literatur (Auswahl)}

- Batagelj, Validimir/Doreian, Patrick/Ferligoij, Anuska und Kejzar. Natasa (2014): Understanding Large Temporal Networks and Spatial Networks. Exploration, Pattern Searching, Visualization and Network Evolution. Chichester (Wiley).

- Bourdieu, Pierre (2000): Sozialer Raum und Klassen. F. a. M. (Suhrkamp).

- Ders.: (2005): Ökonomisches Kaptal - kulturelles Kapital - Soziales Kapital. In: Die verborgenen Mechanismen der Macht. Schriften zu Politik \& Kultur 1. Hamburg (VSA).

- Burt, Ronald S. (1995): Structural Holes. The Social Structure of Competition. Harvard University Press.

- Ders.: Brokerage and Closure (2007): An Introduction to Social Capital. Oxford University Press.

- Emirbayer, Mustafa (1997): Manifesto for a relational sociology. American Journal of Sociology, Vol. 103, S. $281-317$.

- FAS.research, Rat für Forschung und Technologieentwicklung (Hrsg.) (2005): Exzellente Netzwerke; Wien.

- FASresearch et al. (2008): Netzwerke der Wissensproduktion. Eine Studie der FASresearch im Auftrag von RFTE, FFG und w-fFORTE. Wien.

- Fath, Brian D./Dean, Carly/Katzmair, Harald (2015): Navigating the adaptive cycle: an approach to managing the resilience of social systems. Ecology and Society 20 (2): 24 [online] URL: http://www.ecologyandsociety.org/vol20/iss2/art24/, abgerufen am 17.07.2015.

- Florida, Richard (2002): The Rise of the Creative Class. New York (Basic Books).

- Gunderson, Lance H./Holling, C.S. (2002): Panarchy. Understanding Transformations in Human and Natural Systems. Washington, Covelo, London (Island Press). 
- Jansen, Dorothea (2003): Einführung in die Netzwerkanalyse. Grundlagen, Methoden, Forschungsbeispiele. Wiesbaden (VS Verlag für Sozialwissenschaften).

- Katzmair, Harald (2012): Soziales Kapital und Resilienz. Adaptive Netzwerke in einer Welt der „schöpferischen Zerstörungen”. In: Wirtschaftspolitische Blätter 1/2012, S. $141 \mathrm{ff}$.

- Ders. (2014): Alles hat seine Zeit. Resilienz in sozio-ökologischen Systemen und ihre Rolle in der (Umwelt-) Didaktik. FORUM Umweltbildung, BNE-Jahrbuch 2014.

- Krempel, Lothar (2005): Visualisierung komplexer Strukturen. Grundlagen der Darstellung mehrdimensionaler Netzwerke Frankfurt / New York (campus Verlag).

- de Nooy, Wouter, Mrvar Andrej und Batagelj, Vladimir (2011): Exploratory Social Network Analysis with Pajek. Cambridge (Cambridge University Press).

- Rogers, Everett M. (2003): Diffusion of Innovations, New York (Free Press).

- Schumpeter, Joseph A. (2005): Kapitalismus, Sozialismus und Demokratie. Stuttgart (UTB).

- Thompson, Michael/Ellis, Richard/Wildavsky, Aaron (1990): Cultural Theory. Westview Press.

- Thompson, Michael (2008): Organising and Disorganising. A Dynamic and Non-Linear Theory of Institutional Emergence and its Implications. Triarchy Press.

- Unterlass, Fabian/Hranyai, Kathrin/Reinstaller, Andreas (2013): Patentindikatoren zur Bewertung der erfinderischen Leistung in Österreich. Vorläufiger technischer Bericht. Österreichisches Institut für Wirtschaftsforschung, Wien 2013.

- Vedres, Balász/Stark, David (2010): Structural Folds: Generative Disruption in Overlapping Groups. In: AJS American Journal of Sociology, Volume 115 Number 4 (January 2010): 1150-90. 
- Wasserman, Stanley und Faust, Katherine (1994): Social Network Analysis: Methods and Applications. Cambridge (Cambridge University Press). 